\title{
EXPLORING CHANGES IN FUNCTIONAL STATUS WHILE WAITING FOR TRANSCATHETER AORTIC VALVE IMPLANTATION
}

by

Jacqueline Marie Forman

BScN, The University of Alberta, 1996

\begin{abstract}
A THESIS SUBMITTED IN PARTIAL FULFILLMENT OF
THE REQUIREMENTS FOR THE DEGREE OF
\end{abstract}

MASTER OF SCIENCE IN NURSING

in

THE FACULTY OF GRADUATE STUDIES

(Nursing)

THE UNIVERSITY OF BRITISH COLUMBIA

(Vancouver)

August 2013

(C) Jacqueline Marie Forman, 2013 


\section{Abstract}

As the body ages, there is a natural decline in physical and cognitive abilities. The presence of chronic disease can accelerate this process. Aortic stenosis (AS) is a structural heart disease primarily associated with aging. Untreated patients die within 2 to 5 years following the onset of symptoms. For individuals with multiple co-morbidities, surgical treatment is not an option because of high risk for surgical complications. An innovative and minimally invasive procedure called transcatheter aortic valve implantation (TAVI) has emerged as a safe and viable treatment option for higher risk patients. Because of the rapid disease progression of severe AS and the varying wait-times prior to procedure, it is important to understand changes in functional status while waiting for TAVI. The purpose of this study was to examine the changes in functional status between time of eligibility assessment and TAVI procedure date. Changes in functional status including 5-Metre Gait Speed, Canadian Study of Health and Aging Clinical Frailty Scale and Mini Mental State Examination were evaluated using an exploratory prospective cohort design.

Thirty two patients participated in the study with median age 81 (range 64 to 93). Functional status declined between time of assessment and time of TAVI: Gait speed increased by 0.53 seconds $(p=0.01)$ and Clinical Frailty Scale increased by 0.31 (from 4.3 to 4.6, $p=0.01)$. Patients who waited longer than six weeks for TAVI $(n=19)$ had a larger decline in gait speed than patients who waited less than six weeks $(n=10)(0.8 \mathrm{sec}$ vs 0.0 sec, $p=0.04)$. Patients who were living alone $(n=11)$ had a larger increase in frailty scores compared to patients living with another adult $(n=21)(0.6$ vs $0.1, p=0.05)$.

This study has shown that change in functional status may be an important assessment to monitor while patients are waiting for TAVI. Results may be used to facilitate 
individualized care and management strategies and inform health care policy to develop evidence based benchmarks for safe wait-times. Future research with larger samples could validate the exploratory findings of this study. 


\section{Preface}

Ethics approval was granted by the University of British Columbia Providence Health Care Research Ethics Boards (REB) with certificate number UBC-PHC H12-01637. Ethics approval was also provided by the Vancouver Coastal Health Research Institute with certificate number V12-01637. 


\section{Table of Contents}

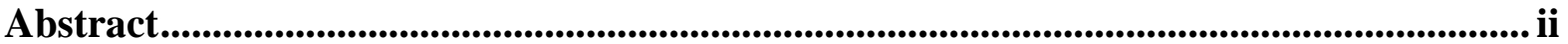

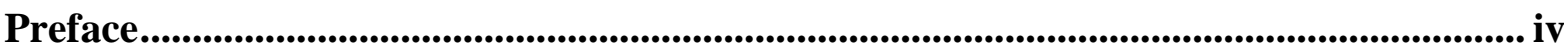

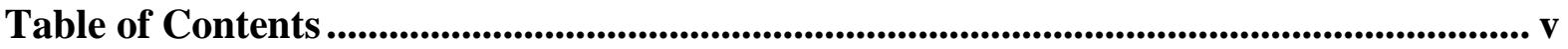

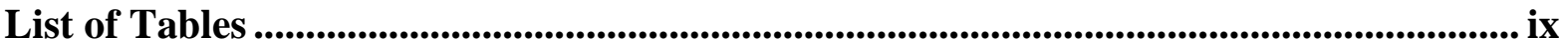

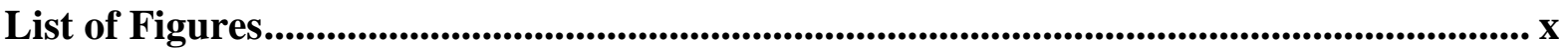

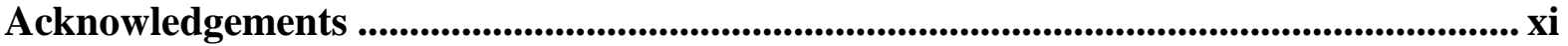

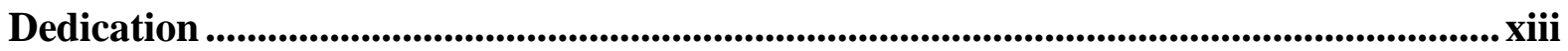

Chapter 1: Introduction ............................................................................................................................. 1

1.1 Diagnosis of Aortic Stenosis and Treatment Options ......................................... 2

1.2 Transcatheter Aortic Valve Implantation....................................................... 3

1.3 Vancouver Transcatheter Heart Valve Program ................................................ 3

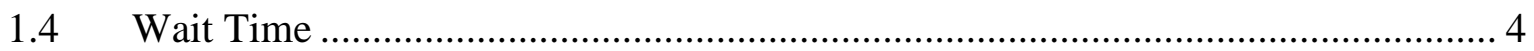

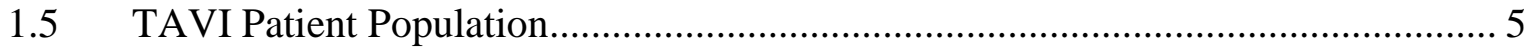

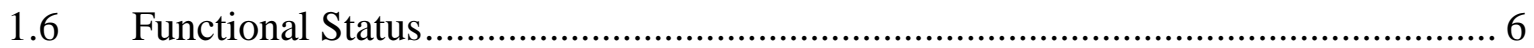

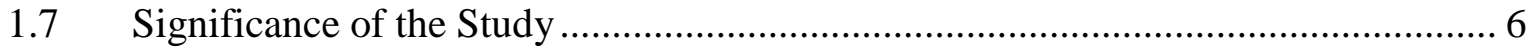

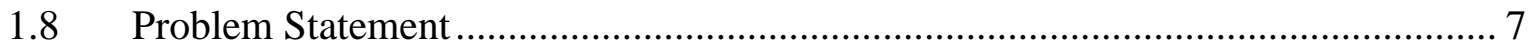

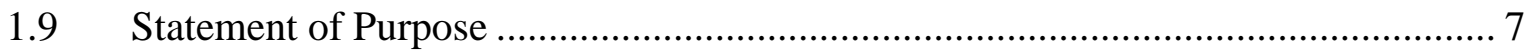

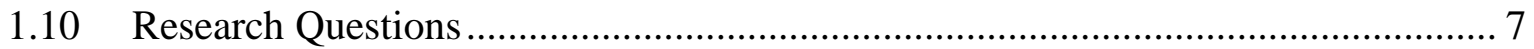

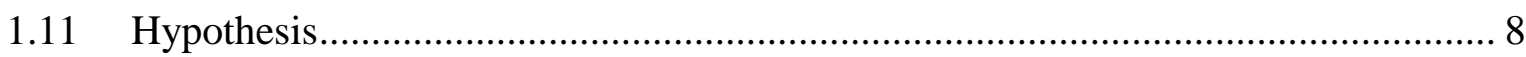

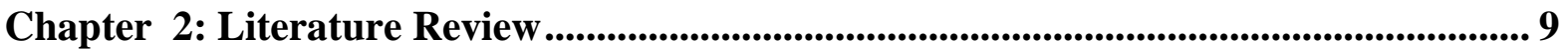

2.1 Clinical and Functional TAVI Patient Outcomes ............................................ 9

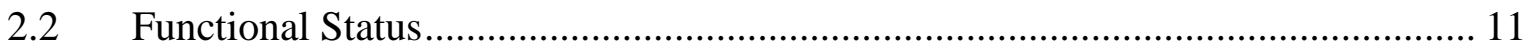


2.3 Functional Status Measures in TAVI Research ................................................ 15

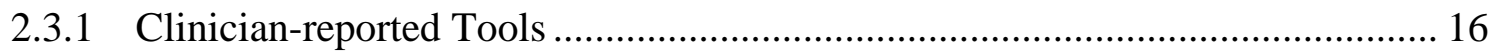

2.3.1.1 The New York Heart Association Classification Tool ................................ 16

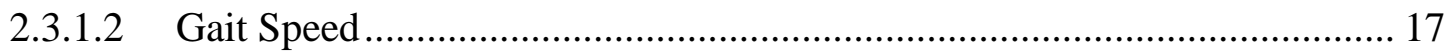

2.3.1.3 Mini-Mental State Examination................................................................. 19

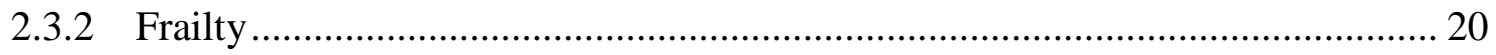

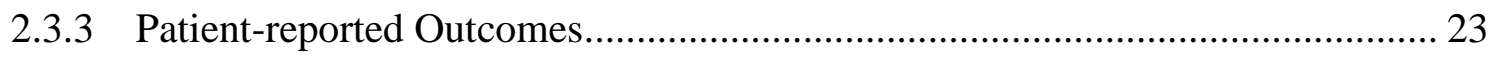

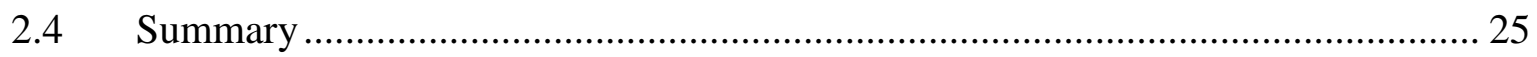

Chapter 3: Methods ................................................................................................................................... 29

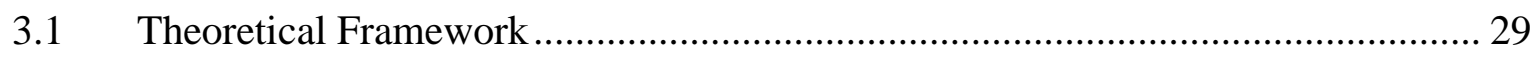

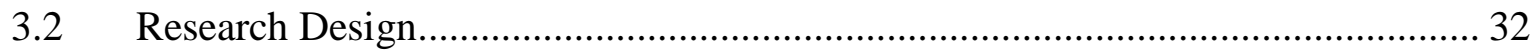

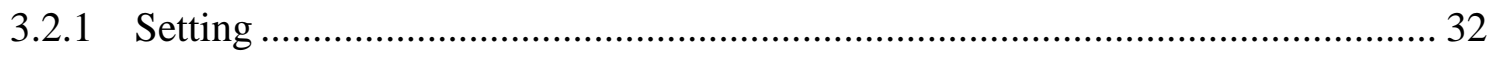

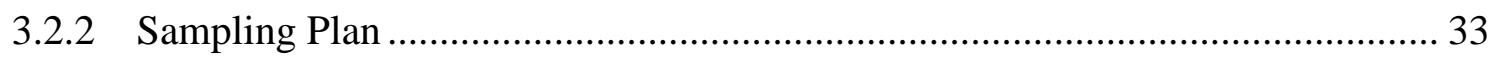

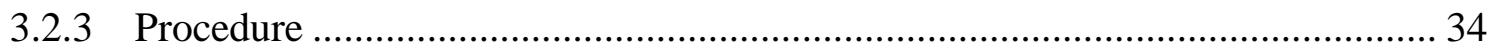

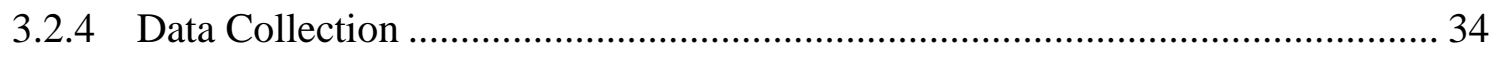

3.3 Operationalization of Study Constructs: Measurements of Functional Status ....... 35

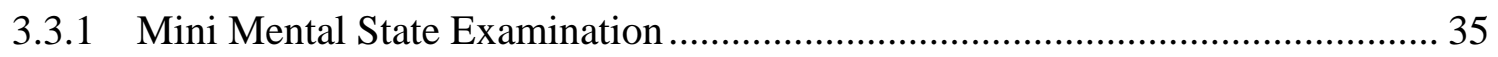

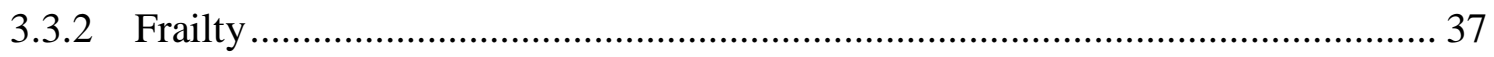

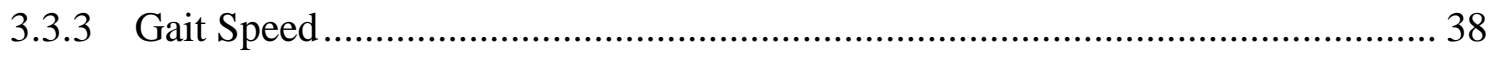

3.4 Operationalization of Study Constructs: Predictor Variables ................................ 40

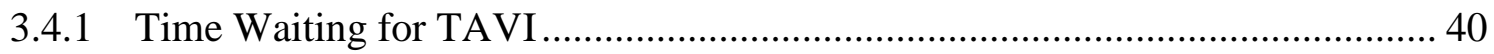

3.4.2 Age

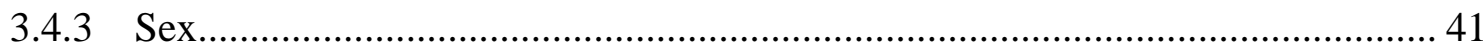




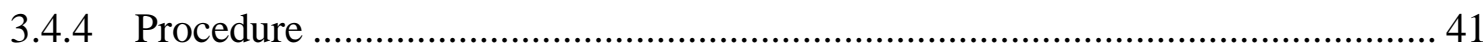

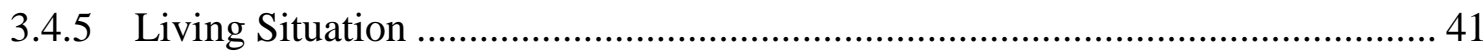

3.4.6 Left Ventricular Ejection Fraction ............................................................... 42

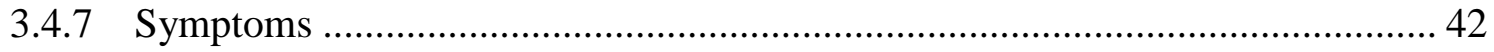

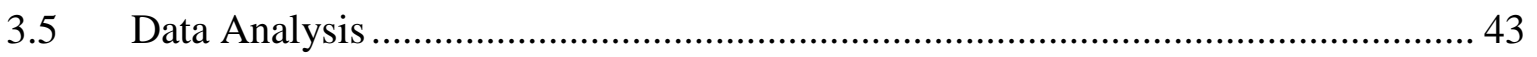

3.6 Ethical Considerations …………………………....................................... 44

Chapter 4: Results.......................................................................................................................................... 45

4.1 Study Recruitment and Enrolment.................................................................... 45

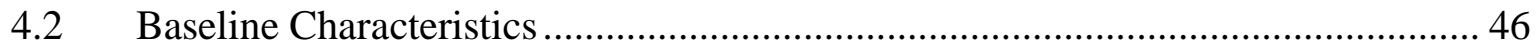

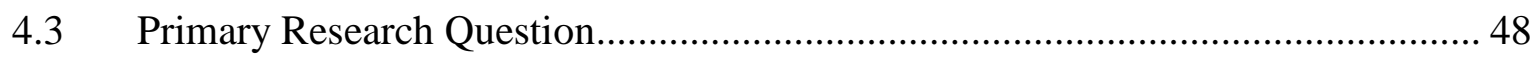

4.3.1 Functional Status Changes .............................................................................. 49

4.4 Secondary Research Question......................................................................... 51

4.4.1 Mean Differences in 5-Metre Gait Speed Test by Predictor Variables ............... 51

4.4.2 Mean Differences in Clinical Frailty Scale by Predictor Variables..................... 54

4.4.3 Mean Differences in MMSE Scores by Predictor Variables .............................. 57

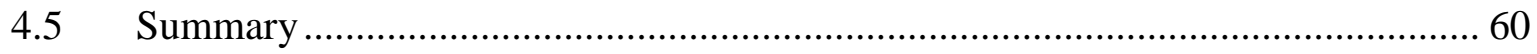

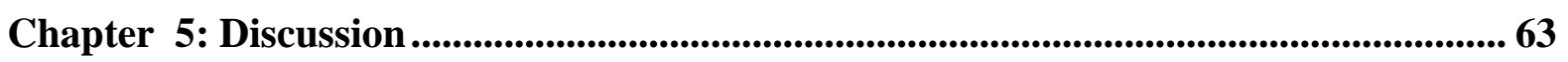

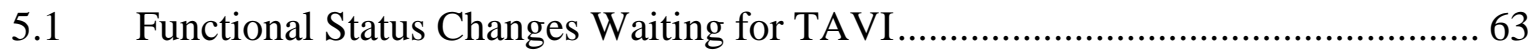

5.1.1 Findings Compared with the Literature …………............................................. 64

5.2 Correlation between Predictor Variables and Changes in Functional Status ......... 65

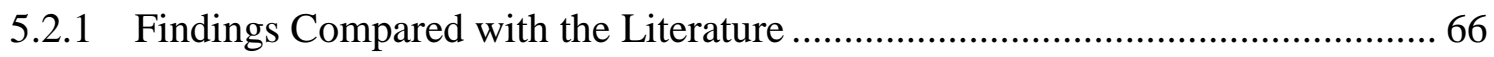

5.3 Findings as they Relate to the Theoretical Framework ......................................... 67

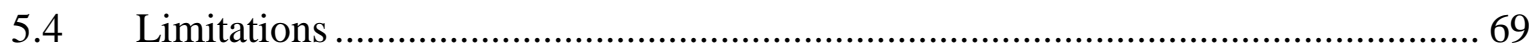




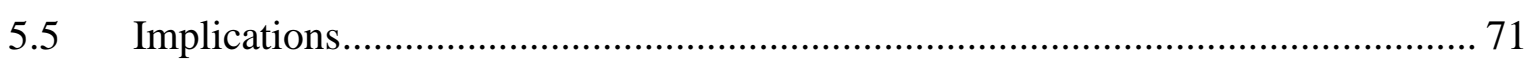

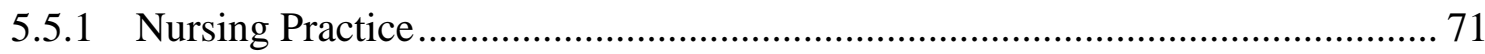

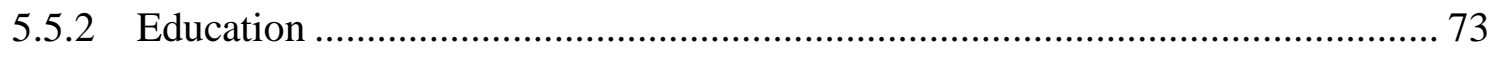

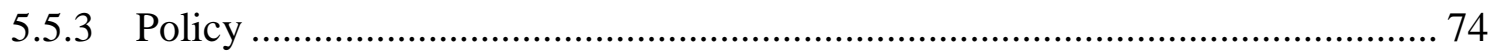

5.5.4 Recommendations for Future Research .............................................. 75

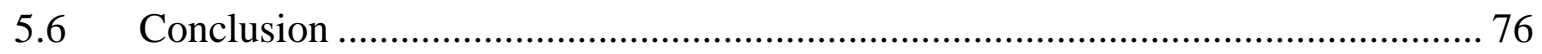

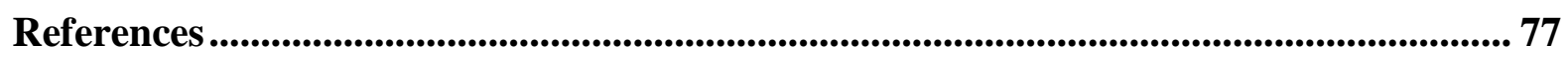

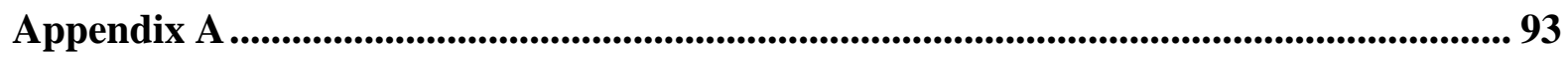

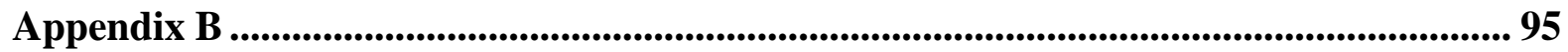

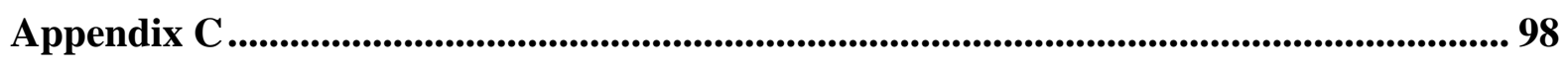

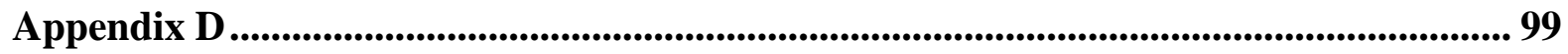




\section{List of Tables}

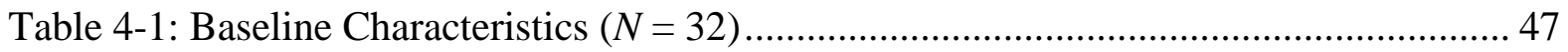

Table 4-2: Functional Status Changes from Eligibility Assessment to Time of Procedure ... 50

Table 4-3: Mean Differences in 5-Metre Gait Speed by Predictor Variable ......................... 53

Table 4-4: Mean Differences in Clinical Frailty Scale by Predictor Variable ...................... 56

Table 4-5: Changes in Clinical Frailty Scale by Age ................................................ 57

Table 4-6: Mean Differences in MMSE Scores by Predictor Variable ............................... 59 


\section{List of Figures}

Figure 3-1: Theoretical Framework ......................................................................... 31

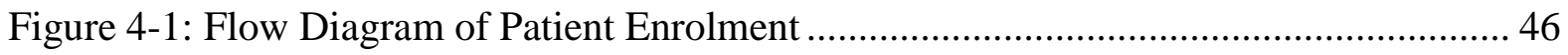

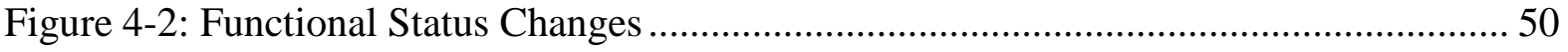

Figure 4-3: Mean Differences in 5-Metre Gait Speed Test and Age .................................. 54

Figure 4-4: Mean Differences in Clinical Frailty Scale and Age ...................................... 57

Figure 4-5: Mean Differences in MMSE Score and Age .............................................. 60 


\section{Acknowledgements}

It is with immense gratitude that I would like to acknowledge the many individuals and organizations that have provided me with support, encouragement, and learning opportunities through the journey of completing this study. I would like to thank the patients who consented to participate in this study. Their enthusiasm and interest in taking part in such a novel study was a truly rewarding experience for a novice researcher. Leslie Achtem and Kristin Kernaghan of the Vancouver Transcatheter Valve Clinic played an enormous role in my completing this study. They were always willing to share their time and knowledge of the clinic processes and were committed to providing assistance with study enrollment. I would also like to thank the staff of the Pre-Admission Clinics for facilitating a seamless process of data collection and a special thanks to Arvinder Buttar for her contributions as research assistant.

I wish to thank my committee chair, Dr. Jennifer Baumbusch, for sharing her passion, knowledge and expertise in the field of geriatric care. I am also appreciative of the support and guidance received from the other members of my committee; Dr. Leanne Currie and Dr.

Sandra Lauck. The mentorship and clinical expertise provided by Dr. Lauck and commitment and enthusiasm for nursing research by Dr. Currie made for a extraordinary team that enhanced the quality of my work.

I am grateful for the research grant provided by the Canadian Council of Cardiovascular Nurses and the UBC School of Nursing's Katherine McMillan Director's Discretionary Fund. 
Finally, I would like acknowledge my family for their unwavering support and encouragement and always believing in me. My husband, William and children, Benjamin and Robert, I would like to thank for their patience, understanding and unconditional love. 


\section{Dedication}

This is dedicated to my most loyal and devoted study companions, Jake and Elwood. 


\section{Chapter 1: Introduction}

For an individual experiencing the progressive changes of aging (Cucinotta, 2007), health and functional status can be significantly altered by the addition of a chronic disease or illness (Boyd, Weiss, Halter, Han, Ershler \& Fried, 2007). Aortic stenosis (AS) is a disease which causes progressive calcification or thickening of the aortic valve. It is found predominantly in the elderly and when symptoms become severe, the disease progresses rapidly causing death among untreated patients (Ross \& Braunwald, 1968).The gold standard treatment for severe AS continues to be conventional open heart surgery to replace the aortic valve (Leon et al., 2010). For individuals who are elderly and who have multiple comorbidities however, surgical treatment is not an option because they are deemed too high risk for surgery. An innovative and minimally invasive procedure called transcatheter aortic valve implantation (TAVI) has emerged as a safe and viable treatment option for this high risk population.

In 2005, St. Paul's Hospital began performing TAVI with special access permission from Health Canada. Since that time, the Canada Food and Drug Act approved a first generation Edwards SAPIEN transcatheter heart valve for patients with severe, AS and deemed non-operable (Health Canada, 2012). As a specialty procedure requiring highly trained operators and allied health staff, TAVI is currently only available at four different sites within British Columbia (BC). Funding is provided by Cardiac Service British Columbia (CSBC), an agency of the Provincial Health Services Authority based on patient requirements and emerging evidence. In 2012/13, 230 TAVI procedures were performed in BC (S. Lauck, personal communication, July 16, 2013). Although TAVI programs are growing in volume, and dynamic changes to improve access to services continue, patients 
must wait varying amounts of time between when they are identified as candidates and when they receive the procedure. Exploring the changes in functional status of this high risk, elderly population waiting for TAVI is critical, as the paradigm shift in the treatment for AS continues to evolve.

\subsection{Diagnosis of Aortic Stenosis and Treatment Options}

Aortic stenosis (AS) is a disease found predominately in the elderly and is considered the most common valve disease in adults over 60 years of age (Gongidi \& Hamaty, 2011). In a population-based study by Nkomo et al. (2006), the reported prevalence of AS for people over 75 years of age was $2.8 \%$ and is expected to rise as our population ages. AS can be caused by an inflammatory process from previous rheumatic heart disease, congenital malformation, or more commonly, degenerative calcification (Madden \& Hill, 2009). At the onset of stenosis, the disease may go undiagnosed because many individuals do not exhibit any symptoms. As the stenosis progresses and valve leaflets become more restricted, blood flow exiting the heart begins to diminish and individuals become increasingly symptomatic. The symptoms of AS may include shortness of breath, angina, fainting and heart failure (Gongidi \& Hamaty, 2011; Maganti, Rigolin, Sarano, \& Bonow, 2010).

In addition to the presentation of symptoms and clinical assessment, a diagnosis of AS can be confirmed with cardiac imaging, including echocardiography or cardiac catheterization. The only effective treatment which provides symptom relief and improves long term survival is surgical aortic valve replacement (AVR) (Holmes et al., 2012). The decision to proceed with surgical AVR is determined by valve biological functioning, presenting symptoms and risk of mortality during cardiac surgery. For individuals deemed 
not suitable for surgery, alternative treatment options are considered such as medical therapy or TAVI.

\subsection{Transcatheter Aortic Valve Implantation}

For individuals who are non-surgical candidates because of the high risk for procedural complications and post-operative mortality, TAVI has emerged as a therapeutic treatment option showing favorable short and longer term outcomes such as improved clinical symptoms and hemodynamic function of the new valve (McRae \& Rodger, 2012; Toggweiler et al., 2013). The first in-human transcatheter aortic valve was implanted in Europe in 2002 (Cribier et al., 2002). TAVI is considered minimally invasive because unlike conventional aortic valve replacement, which requires an open sternotomy, this procedure uses a catheter based stent valve that is deployed in the aortic position while the heart is still beating, without the use of a cardiopulmonary bypass machine. Access to the aortic valve is most commonly achieved with a femoral artery approach and can be performed under general or local anesthesia. Less frequently used access sites include the axillary artery, ascending aorta or transapically through the apex of the left ventricle, all of which are performed by general anesthesia (Webb, 2012).

\subsection{Vancouver Transcatheter Heart Valve Program}

Since the first in-human TAVI implantation in 2002, there has been rapid growth in the use of this procedure along with multiple research and technological advances to support this practice. Reported one year outcomes of TAVI for inoperable patients have consistently demonstrated improved survival rates and quality of life outcomes (Webb, 2012). At St. Paul's Hospital in Vancouver, (Webb, 2012) over 750 patients have been treated with TAVI 
since their first implant in 2005. The current uncertainty about health care funding models and emerging TAVI evidence makes it difficult to predict the anticipated wait time.

In September of 2011, the TAVI program at St. Paul's Hospital began performing lower risk TAVI at Vancouver General Hospital to increase the program's capacity to ensure timely patient access to treatment. As one combined program, the Vancouver Transcatheter Heart Valve (THV) clinic is able to ensure standardized processes for procedure indications and medical practice, the provision of evidence informed care and increase the accessibility of TAVI for British Columbians. The THV clinic receives approximately thirty referrals for TAVI per month. When a referral is accepted by the program, a variety of diagnostic tests, a clinical assessment visit (cardiology and nursing) and surgical consultation are scheduled over a two-day period as part of the work-up process. The clinical visit is completed by the program nurse coordinator and includes a global functional assessment developed by the multidisciplinary THV clinic team. This global assessment includes physical, cognitive, and social components of functional status. When the diagnostic findings, clinical assessment and consultation reports are available, the THV team determines if the patient is eligible for the procedure.

\subsection{Wait Time}

In 2012, the wait-time for TAVI from time of eligibility assessment until procedure date at the Vancouver THV clinic was two to six months depending on clinical factors (S. Lauck, personal communication, September, 2012). A total of 16 procedures are performed monthly in the program with a usual distribution of 14 at St. Paul's Hospital and two at Vancouver General Hospital. Time on a wait list can be affected by vascular access, native valve anatomy, suitability for available valves or operational challenges. In the absence of 
evidence to support specific maximum wait times for TAVI, CSBC has developed an Urgency Rating Score with recommended wait time for all heart surgeries. The current Urgency Rating Scores in British Columbia include emergency status, and three levels of priority ranging from a recommended wait-time of three days for Priority I to three months for a Priority III assignment. Priority scores are based on individual co-morbidities, coronary anatomy and functional characteristics (CSBC, 2012). TAVI is primarily performed as a planned and elective Priority II procedure.

\subsection{TAVI Patient Population}

Between 2005 and 2012, at St. Paul's Hospital, the average age of a patient undergoing TAVI was 82 years (S. Lauck, personal communication, June, 2012). In this elderly population with severe AS, chronic conditions and co-morbidities are commonly present (MacKnight \& Rockwood, 2001; Stortecky et al., 2012; Vahanian et al., 2008). These chronic conditions can lead to loss of independence, decreased functional capacity and isolation (McPherson \& Wister, 2008). It is critical to recognize the multiple, associated comorbidities and heterogeneity in this aging population as it can help identify potential risk factors and individualize care (Boyd et al., 2007; Maddox, 1987). In addition to the associated high-risks due to co-morbidities, patients undergoing TAVI are also more likely to be deemed frail (Rodes-Cabau \& Mok, 2012).Frailty has recently emerged in the literature as an important component of overall health status and clinical indicator of decision-making in TAVI patients (Green et al., 2012). Frailty (described in Section 3.3.2) is a syndrome characterized by muscle weakness, diminished energy reserves, decreased physical and social activity, weight loss and slow gait (McPherson \& Wister, 2008). Frail, elderly individuals 
waiting for TAVI are at considerable risk for changes in functional status in the presence of severe AS and associated co-morbidities.

\subsection{Functional Status}

Recently, there has been a paradigm shift in the management of cardiac care of older adults due to the identification of a fundamental link between (a) the treatment of cardiac disease in the elderly and (b) factors such as; co-morbidities, frailty, functional limitations and cognitive decline (Forman et al., 2011). Functional status generally refers to one's ability to fulfill usual roles and activities of daily living in all domains of life including physical, psychological, social and spiritual (Leidy, 1994; Wang, 2004). The importance one places on quality of life and functional status varies with each individual (Wilson \& Cleary, 1995); therefore we ought to be cognizant of the value to which individual's place on their ability to

complete activities of daily living. Utilizing measures of functional status in conjunction with standard clinical and diagnostic criteria could improve risk stratification and help inform TAVI patient selection. In addition, a comprehensive assessment of functional status prior to undergoing TAVI provides valuable baseline information and has shown to be an important predictor of morbidity and mortality outcomes (Schoenenberger et al., 2012).

\subsection{Significance of the Study}

For patients waiting for TAVI at the Vancouver THV clinic, there is a varying length of wait-time between the eligibility assessment and date of procedure. Throughout this varying wait-time, multiple factors can cause changes in functional status. In a population more likely to live alone and have increased co-morbidities, and other health vulnerabilities it is critical to explore changes to functional status while waiting for TAVI. Progressive worsening of symptoms and disease pathology can also lead to significant changes in an 
individual's global level of functioning. For an elderly individual with co-morbidities, rapid progression of AS symptoms while waiting for TAVI can have detrimental consequences including mortality (Madden \& Hill, 2009). Exploring the changes in functional status of individuals waiting for TAVI is critical in helping to inform care and improve patient management during this period of potentially rapid disease progression and associated changes to function.

\subsection{Problem Statement}

In the Vancouver THV program, the functional status assessment for TAVI occurs at the time of eligibility assessment which, during this study, took place approximately two to six months prior to the procedure being performed. For an elderly individual, the presence of co-morbidities, worsening symptoms and other influencing factors could significantly contribute to changes in function during that wait period. As our population ages, the prevalence of AS is expected to rise (McRae \& Rodger, 2012). With an increase in patient referrals, and the potential for rapidly deteriorating health with severe AS, it will become increasingly important to examine the relationship between wait-time and other influencing factors which have a causal effect on functional status to inform clinical care and health care policy.

\subsection{Statement of Purpose}

The purpose of this research is to describe the changes in functional status between time of eligibility assessment and the TAVI procedure date.

\subsection{Research Questions}

1. In individuals with severe AS, does functional status change from the time of eligibility assessment until the time of procedure? 
2. In individuals with severe AS, what predictor variables correlate with changes in functional status while waiting for TAVI?

\subsection{Hypothesis}

1. During the wait-time between eligibility assessment and TAVI procedure, individuals with severe AS will experience a decline in their functional status.

2. There is a relationship between selected predictor variables such as length of waittime, age, sex, living situation, procedure, biological function, reported symptoms and changes in functional status while waiting for TAVI. 


\section{Chapter 2: Literature Review}

In order to provide clarity and context to the task of exploring functional status in the TAVI population, it is important to appraise the literature and report any gaps identified. The purpose of this chapter is to review findings of TAVI research, provide an analysis of functional status and describe currently used measures of functional status in TAVI. To begin, a review of clinical and functional TAVI patient outcomes is provided, followed by a thorough analysis of functional status found in the literature. The subsequent section provides a review of currently used measures of functional status in TAVI research which includes clinician-reported tools, frailty and patient-reported outcomes. Finally, a summary and gaps identified within the TAVI literature are examined.

The review for this study entailed an extensive search of the literature using the Cumulative Index of Allied Health and Nursing Literature (CINAHL), PubMed, Embase and Google Scholar databases. The keywords included: transcatheter aortic valve replacement, implantation, percutaneous aortic valve replacement, implantation, elderly, frailty, functional status and quality of life. The inclusion criteria included: study participant age over 65, studies within the past ten years (2002-2012), and human subjects only.

\subsection{Clinical and Functional TAVI Patient Outcomes}

Since the first implantation in 2002, multiple studies have been conducted to examine clinical and functional outcomes of TAVI. For inoperable patients with severe AS, TAVI has demonstrated favorable outcomes. In 2010, results of a large randomized control trial demonstrated the safety and efficacy of TAVI and a $20 \%$ absolute reduction in one-year mortality in patients otherwise deemed inoperable (Leon et al., 2010). The two year follow up from this same trial was subsequently reported in 2012 and showed decrease rates of 
death, hospitalization, and improved valve hemodynamics (Makkar et al., 2012). Very few studies have reported outcomes greater than one to two years due to the limited time TAVI has been available to treat severe AS and the advanced age of TAVI patients. However, Toggweiler et al. (2013) recently reported favorable outcomes demonstrating a five year survival rate of $35 \%$ which is the longest period of time outcomes have been measured following TAVI.

Since the first transcatheter implantation, there has been a growing body of literature in the area of TAVI as researchers attempt to explore the efficacy and safety of this innovative procedure (Canadian Agency for Drugs and Technologies in Health, 2013). There have been a large number of studies conducted to measure mortality, morbidity and clinical function however; very few have examined functional status as a study end point (Gotzmann et al., 2011; Reynolds et al., 2012; Reynolds et al., 2011; Schoenenberger et al., 2012).

As TAVI becomes increasingly accessible and indication for its use expands, it is imperative for research to include measures of functional status as primary endpoints to guide case selection and inform indications. In a predominantly elderly population, changes in functional status can have detrimental effects to level of independence, ability to fulfill daily roles and ultimately affect quality of life (Leidy, 1994). Further, the trajectory of functional abilities in this vulnerable population is relatively unknown and may be exacerbated by the progression of AS, comorbid conditions and severity of symptoms. It is imperative for TAVI research to include functional status as a prognostic tool to improve risk stratification and provide valuable baseline data in which to compare post-procedures outcomes. The challenge also lies in determining an operational definition for functional 
status and consistent measurement tools used in TAVI research. These issues are examined in the following sections.

\subsection{Functional Status}

As reported in the previous section, a significant gap in the literature is a lack of consensus regarding an operational definition of functional status. Without a clear understanding of functional status, the challenge lies in choosing appropriate measurement tools, analyzing inconsistent research outcomes and the inability to provide meaningful clinical support. Adding to the complexity of defining functional status is that terms such as 'functional ability', 'functional outcomes', 'health status 'and 'quality of life' are often used interchangeable (Knight, 2000; Leidy, 1994). This section will provide a review of the literature related to functional status and the various measurement tools and definitions used to operationalize functional status within TAVI research.

The literature shows researchers recognize the importance of measuring functional status in this vulnerable, elderly population, however there are inconsistencies related to defining this concept. A variety of measurement tools have been used to describe functional status in the TAVI literature. In a 2011 study reporting one-year TAVI outcomes, Thomas et al. (2011) used the term functional status to describe the patient's New York Heart Association (NYHA) classification score. Bagur et al. (2011) used results of the Duke Activity Status Index (DASI), a self-administered questionnaire which evaluates abilities to perform daily activities, as a measure of combined functional status and quality of life.

Gotzmann et al. (2011) described significant clinical improvements in functional status one year after TAVI by using the NYHA classification system and administration of patient- 
reported questionnaires, but used the terms 'functional status', 'functional outcome' and 'functional capacity' interchangeably when reporting results.

Functional status assessment was originally developed in rehabilitation medicine to provide a means of measuring physical performance related to disabilities as reported by the ability to perform daily living activities (Wang, 2004). Since that time, a variety of definitions have been reported within the health disciplines literature. Katz, Ford, Moskowitz, Jackson and Jaffe (1963) developed a measurement tool which would assess functioning in the elderly and chronically ill. The items addressed in this index of activities of daily living (ADL) include the independent ability to bath, dress, toilet, transfer, feed and continence. Describing functional status in terms of the ability or inability to perform daily activities directs focus solely on one's performance or behavior (Knight, 2000; Wang, 2004). Bennett, Reigel, Bittner and Nichols (2002) reported functional status as an individual's ability to function in regular, daily activities and can be influenced by cognitive, social and environmental factors. In the study by Bennett et al. (2002), functional status was viewed as an accurate measurement of one's ability to perform daily activities, however, they recommend use of the NYHA classification system which is a clinician-reported tool based on clinical judgment instead of patient-reported score generated by the activities of daily living index.

Viewing functional status with a broader, comprehensive approach which incorporates self-management skills, cognition, and emotional components has also been reported in the literature. Moinpour, McCorkle, and Saunders (1988) described functional status with a holistic approach and proposed that an accurate measurement of functional status would address one's functioning in areas of "physical health, quality of self- 
maintenance, quality of role activity, intellectual status, social activity, attitude toward the world and toward self and emotional status" (p. 24). Wang (2004) described functional status as activities of daily life performed by an individual to meet the needs within all aspects of life including "physical, psychological, social, spiritual, intellectual and roles" (p. 462). This definition would imply a measurement tool that does not include multiple, holistic components, would not truly capture a comprehensive assessment of functional status. The importance of including cognitive function in the dimension of functional status has also been reported. Knight (2000) posits caution must be taken when more specific activities of daily living are used to assess functional status because the researcher assumes the individual is cognizant or knows what to do. When the instrumental activities of daily living (IADL) index tool is used, individuals are given a score if they are independent with using the telephone, shopping, food preparation, housekeeping, laundry, transportation, medications and finances (Lawton \& Brody, 1969). By asking an individual if they can manage their finances, we are assuming an underlying cognitive ability in that they know how to perform or problem solve a task. The end result may still indicate an inability to perform a task; however, recognizing the relationship between cognitively impaired or functional limitations due to symptoms is imperative if we are going to use results to improve clinical practice. Leidy (1994) also referred to functional status as encompassing a larger domain of functioning which would include physical, spiritual, social and psychological domains. In an effort to define and clarify the concept of functional status, Leidy developed an analytical framework which includes capacity, performance, reserve and capacity utilization as dimensions of functional status. Functional capacity refers to the maximum potential an individual has to perform physical and psychosocial activities. An assessment of one's 
cognitive abilities would be an example of capacity. The activities of daily living and instrumental activities of daily living are examples of functional performance which help determine what an individual chooses to do. She further describes functional reserve as the difference between capacity and performance and assumes that individuals may not perform to their full potential on a daily basis. Finally, capacity utilization describes the extent to which an individual may be required to use their full capacity to complete daily activities. This can be seen with an individual who experiences symptoms of fatigue and may be required to expend extra 'energy' to complete daily activities (Leidy, 1994). The notion of functional capacity and performance were also reported in a study by (Bennett et al., 2002). However, rather than viewing capacity and performance as dimensions within functional status, they see them as separate concepts. Functional status is defined as an individual's ability to perform regular activities potentially limited by external and internal factors including symptoms. Functional capacity assesses the maximum function in which an individual is capable and performance relates to the level of activity chosen by the individual (Bennett et al., 2002).

The literature has shown functional status to be a concept which has multiple definitions and methodologies to measure outcomes. Leidy (1994) reported researchers and practitioners were on a "functional status merry-go-round" (p. 196) in the absence of a clear definition and continued use of interchanging terminology related to function. Without a clear definition of functional status, challenges will remain in choosing appropriate measurement tools, standardizing TAVI outcomes, and developing a truly patient-centered approach to care. The European Valve Academic Research Consortium described the importance of measuring clinical benefits such as functional status but recognized the bias 
and weakness that accompanied the variety of measurement tools and overall lack of standardization (Leon et al., 2011). The Canadian Cardiovascular Society also encouraged the use of an "objective evaluation of neuro-cognitive function and frailty" to evaluate TAVI candidates (Webb et al., 2012, p. 7). Without providing an operational definition, a consensus statement endorsed by the American Heart Association (AHA) recommended functional status be included when assessing the elderly for TAVI eligibility (Holmes et al., 2012). The importance of assessing functional status is currently being recognized by TAVI researchers but if differing definitions remain, it is critical to identify the distinction between the various components of function and select the most appropriate measurement tool.

Despite the absence of a standardized definition in the literature it is clear that functional status is multidimensional and encompasses an individual's ability to perform daily tasks within all domains of functioning. To obtain this comprehensive assessment of functional status, multiple validated tools to measure varied components of functioning may be required. Researchers could achieve a more precise investigation of functional status by clearly defining the components of functioning being assessed and utilizing a combination of validated tools which would deliver a more comprehensive and multidimensional assessment of functional status. By achieving meaningful, functional status outcomes, we are better prepared to target specific patient management and treatment strategies.

\subsection{Functional Status Measures in TAVI Research}

In reviewing the literature of TAVI outcomes within the past ten years, multiple tools and measurements for components of functional status have been used. Without a standardized definition and measurement for functional status however, it is difficult to accurately describe and compare TAVI outcomes. Quality of life and health-related quality of 
life outcomes have been assessed using validated, patient-reported tools. Clinician-reported measurements such as the NYHA classification tool, timing of gait speed and cognitive impairment assessments have also been used. Finally, the notion of frailty and its importance in recognizing predictors of outcomes in the elderly has also become more prevalent in recent TAVI literature (Lee, Buth, Martin, Yip, \& Hirsch, 2010; Leon et al., 2011; Schoenenberger et al., 2012). The following approaches to measuring functional status will be reviewed in the subsequent section below; clinician-reported tools, frailty and patient-reported outcomes.

\subsubsection{Clinician-reported Tools}

To assess functional status outcomes in the TAVI population, clinician-reported tools are commonly employed. The United States Federal Drug Administration (FDA) describes clinician-reported outcomes as assessments which are made by an observer with professional training in the field of measurement being observed (US Department of Health and Human

Services, 2013). These reported outcomes can be based on observation, diagnostics tests, and evaluation from clinical judgment. The clinician-reported tools which will be reviewed include the NYHA classification tool, gait speed and the Mini Mental State Examination.

\subsubsection{The New York Heart Association Classification Tool}

The NYHA classification tool is one of the most widely used clinician-reported tools which evaluates symptoms of heart failure related to activities of daily life (Bennett et al., 2002). In 1963, the AHA first published the classification tool with a number of revisions occurring since that time (Apostolakis \& Akinosoglou, 2007). In an Executive Summary report of chronic heart failure guidelines, the American College of Cardiology and AHA described the usefulness of this functional classification tool but also recognized a physician assessment could be subjective and classes of heart failure could change quickly (Hunt et al., 
2001). Patient symptoms are classified into four categories ranging from no symptoms of shortness of breath and without any physical limitations (Class I), to severe physical limitations and cardiac symptoms at rest (Class IV). Moving to a lower class of symptoms is an indication of improved function. Although the description of classes is well documented and standardized, operationalizing what is actually being assessed is slightly ambiguous. The NYHA has been described as a tool used to assess the effect of heart failure symptoms on exercise intolerance (Apostolakis \& Akinosoglou, 2007), an indication of functional reserve (Miller-Davis, Marden, \& Leidy, 2006) and a system in which heart failure can be classified according to symptom severity and limitations to physical activity (American Heart Association, Classes of Heart Failure section, 2013). Although a clinician-reported tool such as the NYHA classification system can be subjective based on physician assessment and clinical judgment, its use in assessing degree of physical limitations due to symptoms is important to consider with TAVI outcomes.

The NYHA is a commonly used diagnostic tool useful in comparing outcomes in TAVI literature (Goncalves et al., 2011; Gotzmann et al., 2011; Leon et al., 2010; Makkar et al., 2012; Smith et al., 2011; Thomas et al., 2011; Toggweiler et al., 2013) however, it has been reported to correlate poorly with other measures of functional capacity (Rostagno et al., 2000) and is limited in its reliability and usefulness as an outcome measure (Severo et al., 2011). A tool such as the NHYA that lacks objectivity and reliability would be unsuitable to accurately measure functional status.

\subsubsection{Gait Speed}

Measuring an individual's gait speed is another commonly used clinician-reported tool used in TAVI outcomes (Gotzmann et al., 2011; Leon et al., 2010; Smith et al., 2011). 
Gait speed is emerging as an important marker of frailty, measurement of functional performance and exercise capacity. There are a variety of measurement tools for gait speed such as the 6-Minute walk test, 12-Minute walk test and 5-Metre gait speed (van Kan et al., 2009). The aim of each tool is to provide an objective measurement by which gait speed can be assessed by instructing the individual to walk at usual pace. By measuring gait speed within a pre-specified distance, the observer can assess global physical response to exercise and help determine potential outcomes after medical interventions (Brooks, Solway, \& Gibbons, 2003). In a study of community-dwelling adults over the age of 65 years, gait speed was referred to as a reflection of health and functional status and was shown to be associated with survival at five and ten years with a $p<0.001$ (Studenski et al., 2011). Green et al. (2012) described gait speed as a marker of frailty and an important characteristic of functional status which can be used as a predictor of outcomes in an elderly population with AS. Gait speed has also been identified as a predictor of adverse outcomes in the elderly population undergoing cardiac surgery as well as community-dwelling (Afilalo et al., 2010; van Kan et al., 2009).

The manner in which gait speed is analyzed also varies within the literature (van Kan et al., 2009). Whether measuring the distance covered in a specified time, or measuring the time it takes to walk a certain distance, gait speed can be categorized into slow or normal, or can be measured as a continuous variable. In a study by Green et al. (2012) gait speed was reported as being strongly correlated with dependent functional status and was analyzed as a continuous and categorical variable. Although recent literature demonstrates gait speed as a valid tool to measure function (Afilalo et al., 2010; Green et al., 2012; van Kan et al., 2009), for individuals with physical limitations unrelated to their AS symptoms, conducting this test 
alone may not provide an accurate assessment of functional status. Gait speed provides valuable information regarding functional and exercise capacity but ought to be used in conjunction with other measurements of functional status in order to provide a comprehensive, holistic assessment.

\subsubsection{Mini-Mental State Examination}

As reviewed in the previous section, assessment of cognitive ability has also been identified as an important component of functional status. Also, clinician-reported, independent measurements of cognition have not commonly been used in TAVI research. In a study to analyze predictors of functional decline in elderly TAVI patients, Schoenenberger et al. (2012) used the Mini Mental State Examination (MMSE) to measure cognitive ability at baseline and six months following TAVI. First developed in 1975, the MMSE is a thirtypoint questionnaire which screens for cognitive impairment by addressing components of orientation, recall, attention, language, repetition and the ability to follow complex commands. Range of scores describing degree of cognitive impairment vary depending on the literature source, age of the individual, education level, presence of co-existing mental disorders and language spoken. When using the MMSE to predict functional decline in elderly TAVI patients, scores less than 27 out of 30 points were considered to indicate cognitive impairment (Schoenenberger et al., 2012). This differs from the scoring initially described in the MMSE validation studies which reported a score between zero and 23 to be low reflecting cognitive impairment (Folstein, Folstein, \& McHugh, 1975; Folstein, Anthony, Parhad, Duffy \& Gruenberg, 1985). In reviewing the use of clinical diagnostic aids to address the meaning of cognitive impairment, Folstein et al. (1985) concluded a low MMSE score was useful in detecting the need for further evaluation but should not be used to 
make a diagnosis. This is an important statement because it illustrates the significance of utilizing the MMSE to detect changes in cognitive ability at different time points such as baseline, and post TAVI.

Analyzing trends or changes in MMSE scores of TAVI patients provides meaningful results which could be used to develop specific interventions or treatments directed at cognitive abilities. The inclusion of cognitive ability is vital to obtaining a multidimensional assessment of functional status.

\subsubsection{Frailty}

The relationship between frailty and cardiovascular disease in the elderly has becoming increasingly more evident and reported in the literature (Afilalo, Karunananthan, Eisenberg, Alexander, \& Bergman, 2009; Green et al., 2012; Lee et al., 2010). Measuring frailty has also gained importance in TAVI literature and is recognized as an important indicator of mortality and morbidity outcomes, and component of risk assessment (Holmes et al., 2012; Kappetein et al., 2013; Webb, 2012). Widely recognized however, is the inconsistent definition of frailty and variety of frailty indices which are used. This contributes to difficulties in standardizing assessment scores and decreases the potential for objectivity.

Prior to the late 1970 's, frailty was a term rarely used in the medical field. In the rare instances it was used, it described a patient who was weak or fragile (Hogan, MacKnight, \& Bergman, 2003). In 1978, the Federal Council of Aging in the United States defined the term frail elderly as "persons, usually but not always, over the age of 75, who because of an accumulation of various continuing problems often require one or several supportive services in order to cope with daily life" (Hogan et al., p. 4). It was in 1990 that the term 'frail-old' was first listed in the subject index of the American Geriatric Society (Hogan et al., 2003). In 
the past 20 years, there has been a growing amount of literature published with varying descriptions and conceptualizations of frailty. These differing concepts have provided a building block or framework for others to build upon and develop their own concept of frailty.

There have been numerous attempts to define, analyze and describe the concept of frailty but without a clear consensus, it has been used loosely to describe a range of cognitive and physical disabilities in the elderly population (Lally \& Crome, 2007). Afilalo et al. (2009) described frailty as "a geriatric syndrome of increased vulnerability to stressors due to impairments in multiple interrelated systems" (p. 1616). In addition to multiple definitions of frailty, there is also a wide variety of measurement tools and frailty markers which combine to create an index score. Mack (2012) reported more than twenty frailty scales in the published literature that included a variety of multidimensional physical and cognitive components.

In 2004, The Canadian Institutes of Health Research funded Dr. Kenneth Rockwood to construct a valid frailty scale that could be used to predict possible outcomes and interventions (Canadian Institutes of Health Research, 2006). The Clinical Frailty Scale is a visual and descriptive nine point scale which ranges from a score of one being very fit to nine which is the most frail individual (Dalhousie University Faculty of Medicine, 2012). This frailty scale conceptualizes frailty as a "physiological condition characterized by decreased reserve and diminished resistance to stressors, resulting from cumulative decline across multiple physiological systems, and causing vulnerability to adverse outcomes" (Rockwood, 2005, p. 432). In 2005, the Clinical Frailty Scale was compared with other validated measurement tools and was shown to be effective and similar in predicting mortality and the 
need for institutionalization within a five year period (Rockwood et al., 2005). The Clinical Frailty Scale is not currently present in published TAVI outcome literature; however it is used by the Vancouver THV clinic as a component of their global, functional assessment.

Fried et al. (2001) described frailty as a phenotype which could be identified by unintentional weight loss, weakness, self-reported exhaustion, slow gait speed and low physical activity. In a study examining frailty as a predictor of surgical outcomes in an elderly population, independent association with post-operative complications, increased length of hospital stay were found using the five criteria described in the Fried phenotype (Makary et al., 2010). The inclusion of gait speed within Fried's phenotype of frailty is used more frequently in TAVI literature as a component of functional status. In a study by Green et al. (2012), gait speed was identified as an important component of the frailty phenotype and was reported to be an objective measurement tool to predict morbidity and mortality in older adults with AS.

In 2012, Green et al. used a frailty index similar to the phenotype described by Fried et al. (2001) to evaluate the impact of frailty on survival following TAVI. This frailty index is based on the summary score generated with measurements of gait speed, grip strength, serum albumin and the Katz activities of daily living score. Serum albumin was used as the marker for malnutrition and weight loss. Mack (2012) reported the use of this index as a measurement of frailty within the ongoing PARTNER trials. Sometimes referred to as the Columbia Frailty Index, little evidence is available regarding the reliability and validity of this measurement tool.

In a study examining predictors of functional decline in TAVI patients, Schoenenberger et al. (2012) also recognized the importance of frailty as a predictor of 
functional decline in the elderly but utilized multiple measurement tools to develop a frailty composite index. Based on their assumption from existing literature, they included cognition, mobility and nutritional status as components of their frailty index. Measurement tools which were used included; the MMSE to assess cognitive impairment, a Timed Get Up and Go test (TUG) for gait function, Mini Nutritional Assessment (MNA) and the Basic and Instrumental Activities of Daily Living (BADL) (Schoenenberger et al., 2012). By dichotomizing their measurement instruments at standard cut points, a summary score or frailty index was developed. When evaluating a multidimensional geriatric assessment tool to predict morbidity and mortality after TAVI in a 2012 study, this same frailty index was calculated using summary scores of the MMSE, TUG, MNA and BADL (Stortecky et al., 2012). Measuring frailty in the elderly, TAVI population is emerging as an important component of functional status however; the challenge lies in comparing outcomes and standardizing risk stratification when multiple definitions and measurements of frailty are used. If frailty alone is used to provide a measure of functional status, it is imperative that a multidimensional tool is utilized. An example of this is the frailty index described by Schoenenberger et al. (2012) which includes as assessment of cognition, gait function, nutritional status and activities of daily living. Utilizing a multidimensional tool provides a comprehensive approach to evaluating frailty.

\subsubsection{Patient-reported Outcomes}

To measure patient-reported health status, validated tools such as the Kansas City Cardiomyopathy Questionnaire (KCCQ), Minnesota Living with Heart Failure Questionnaire (MLHFQ), Short Form 36-Item Health Survey (SF-36) and the Short Form 12-Item Health Survey (SF-12) have all been used in studying TAVI outcomes (Bekeredjian et al., 2010; 
Georgiadou et al., 2011; Gotzmann et al., 2011; Reynolds et al., 2011; Reynolds et al., 2012). In striving to achieve a patient approach to care, use of these patient-reported questionnaires has become more widely used in health research. Understanding the impact and outcome of alternative treatment approaches such as TAVI, can be achieved by assessing health status from the patient's perspective (Reynolds et al., 2012). The SF-36 and the condensed SF-12 version of questionnaires are used to assess generic health status and address the components of physical and mental well-being (SF-36.org, 2013).

In the absence of a patient-reported outcome tool specific to TAVI patients, the MLHFQ and KCCQ have frequently been used to measure health status in TAVI outcomes (Gotzmann et al., 2011; Reynolds et al., 2011; Reynolds et al., 2012). Both of these patientreported outcome tools are disease specific and designed to address the effects of heart failure on symptoms, physical, social, and psychological limitations, self-efficacy and quality of life domains. In 2009, a study by Supino et al. examined the validity of the MLHFQ compared to the SF-36 when used with 50 patients undergoing valve surgery. Overall, the MLHFQ was reported as being easy to administer with high reliability and validity in the valve surgery population. Compared to the SF-36, a disease specific health quality of life questionnaire such as the MLHFQ provides more valuable information related to treatment intervention. The Supino et al. (2009) study however, measured the validity of the MLHFQ in a general valve surgery population and was not directed to those who may experience symptoms specific to AS. Although important to address the effects of heart failure which is a common symptom of AS, symptoms such as angina and syncope are not measured with the MLHFQ or KCCQ (Garin et al., 2009). 
Patient-reported outcome tools can be an integral component to patient management and the clinical decision-making environment. Choosing the right tool however, is critical in achieving the most accurate and appropriate results. Clinicians need to consider the population they are assessing, decide among generic or disease-specific tools, choose appropriate response formats, and be prepared to treat based on issues addressed in the measurement tool (Snyder et al., 2012). As previously reported, in the absence of a patientreported outcome tool specific to AS, the validity of results found with any other tool used in this population needs to be critically appraised to determine how results will be managed. Patient-reported outcome questionnaires can provide valuable information related to individual perceptions and self-reported quality of life but do not present a comprehensive appraisal of functional status independent of other measurement tools.

\subsection{Summary}

In this chapter, significant studies related to TAVI clinical outcomes were reviewed and the concept of functional status and the multiple operational definitions were examined. Additionally, the multiple tools currently used to measure functional status were reported including clinician-reported tools, frailty and patient-reported outcomes.

To demonstrate the safety and efficacy of this innovative procedure, numerous studies have been conducted examining mortality and morbidity outcomes (Leon et al., 2010; Makkar et al., 2012; Toggweiler et al., 2013). As discussed previously in Section 2.1, functional status ought to be included in the risk assessment prior to TAVI and measured as a procedural outcome. Identifying functional impairments prior to TAVI could provide valuable information to improve risk assessment, manage potential peri-operative and postoperative complications and develop strategies for successful transitions from hospital. 
Obtaining baseline functional status data is also important to accurately compare and analyze outcome measures at different time points following TAVI. For many elderly individuals, functional status and the ability to complete activities of daily living and live independently are of utmost importance following TAVI (Schoenenberger et al., 2012). In this elderly population, functional status outcomes may be as important to measure as clinical and biological function. As technology advances and patient indications for TAVI expand, it is imperative that researchers recognize the gap that exists by not including a multidimensional assessment of functional status in TAVI assessment criteria and outcomes.

A significant challenge for TAVI researchers is the inconsistent use of the term functional status and the lack of a standard operational definition. Section 2.2 included an analysis of functional status and the commonly used meanings and definitions. The use of terms such as 'functional status', functional ability' and 'health status' were frequently used interchangeably in the literature which leads to challenges in evaluating and comparing data. Without criterion in which to define functional status, researchers need to clearly articulate the component of functioning in which they are attempting to measure rather than loosely using the term functional status. As previously reported, functional status is considered to be an individual's ability to complete daily tasks and activities in all domains of life including physical, psychological, social and spiritual. In the absence of a single, validated tool that can measure all the components of functional status, researchers ought to employ the use of two or more tools which would effectively achieve a multidimensional assessment. Based on this literature review, a multidimensional assessment of functional status ought to include a selection of instruments which could measure cognitive ability, functional or exercise capacity and a comprehensive frailty index which includes activities of daily living. 
A review of currently used measures of functional status used in TAVI research was reviewed in Section 2.3. This section described the variation of measurement tools which included clinician-reported tools, frailty and patient-reported outcomes. Clinician-reported tools include measurements of cognitive impairment using the MMSE, gait speed to measure functional performance and exercise capacity, and the NYHA which is commonly used to evaluate limitations in activity due to symptoms of heart failure. Frailty is also a concept which is poorly defined in the literature and commonly used to describe functional status. Frailty has shown to be an important predictor of outcomes following cardiac surgery and TAVI (Green et al., 2012; Lee et al., 2010) but without a standard definition and measurement tool, it is difficult to compare outcomes and develop specific treatment and interventions. Although relatively absent in TAVI outcomes to date, frailty has become an important and valid marker of functional status in cardiac patients and is especially important in TAVI patients which are predominantly an elderly population (Green et al., 2012; RodesCabau \& Mok, 2012). There are a variety of patient-reported outcome tools which have been used to describe functioning and quality of life with self-reported questionnaires. However, in the absence of a measurement tool specific for patients with AS, results may be useless and misleading and a self-reported questionnaire alone does not provide sufficient information to measure functional status.

This literature review has reported TAVI research findings, provided an analysis of functional status and described the numerous tools used to measure functional status within the TAVI research. A significant gap which has been identified is the absence of a standardized definition for functional status and validated measurement tool to capture all domains of functioning. In addition, to obtain a comprehensive functional status assessment, 
researchers ought to employ the use of multiple validated measurement tools. For the purpose of this study, functional status is defined as an individual's ability to fulfill their normal roles and perform activities of daily living in all domains of life including physical, psychological, social and spiritual (Ferrans, Zerwic, Wilbur, \& Larson, 2005; Leidy, 1994; Wang, 2004; Wilson \& Cleary, 1995). The tools used to measure functional status for this study include; the Clinical Frailty Scale, MMSE to measure cognitive ability and 5-Metre Gait Speed to measure functional capacity. These measures were selected because collectively they capture the multi-dimensional components of functional status and are used in routine clinical practice at the Vancouver THV clinic. These measurement tools will be described further in the next chapter. 


\section{Chapter 3: Methods}

The purpose of this chapter is to present the theoretical framework used to guide this study and describe the research methods including the research design, sampling plan, data collection, analysis and ethical considerations. Study constructs such as functional status and the independent covariates chosen to explore variability in functional status changes are operationalized. The instruments chosen to measure functional status include; 5-Metre Gait Speed test, Clinical Frailty Scale and the Mini Mental State Examination (MMSE). Guided by the theoretical framework underpinning the study, the covariates used to analyze variability of functional status change include; sex, living situation, days waiting for TAVI, type of procedure, left ventricular ejection fraction ${ }^{1}$ and NYHA classification score. Finally, ethical considerations important to this study will also be discussed.

\subsection{Theoretical Framework}

This study was guided by the Wilson and Cleary (1995) conceptual model of healthrelated quality of life modified by Ferrans et al. (2005), which posits that biological function, symptoms and individual and environmental characteristics influence functional status.

General health perceptions are generated by the integration of these components which leads to overall quality of life, the final element of the model. For the purpose of this study, general health perceptions and overall quality of life were not explored.

Wilson and Cleary (1995) suggest that measures of health exist on "a continuum of increasing biological, social and psychological complexity" (p. 60). Biological measures and more complex general health perceptions are at opposite ends of the continuum. The model

\footnotetext{
${ }^{1}$ Left ventricular ejection fraction is a measurement of how much blood is ejected from the left ventricle of the heart with each contraction.
} 
proposes a causal and dominant relationship that exists between five conceptually distinct domains. These domains include: (1) physiological and biological variables which include cells, organs and organ systems, which leads to (2) symptoms, followed by (3) functional status which include physical, social and psychological components, and subsequent (4) general health perceptions, and culminating in (5) overall quality of life.

The conceptual model adapted by Ferrans et al. (2005) describes the same five domains, and proposes a multi-directional, causal relationship between individual and environmental characteristics and the outcome variables. Based on an ecological model of health behavior from Eyler et al. (2002), the Ferrans et al. (2005) model categorizes demographic, developmental, psychological and biological factors as characteristics of the individual. Characteristics of the environment include social elements such as interpersonal relationships, societal beliefs and physical factors which can include living location, housing and neighborhood. Characteristics of the individual and environment are important elements to conceptualize because they intersect and influence all five of the major components of patient outcomes which are depicted by the revised model.

Ferrans et al. (2005) illustrate the complex, multi-directional relationships of functional status with all components of the conceptual model (see Figure 3.1). The domains of functional status and description of causal relationships provided a suitable theoretical framework in which to guide this research. Ferrans et al. (2005) view functional status as optimizing an individual's existing function and includes four distinct dimensions. These four dimensions were guided by Leidy's framework of functional status and include (1) functional capacity, (2) functional performance, (3) functional capacity utilization and (4) functional reserve (Leidy, 1994). As discussed in the previous chapter, functional capacity is 
one's ability to perform physical, social, psychological and cognitive tasks to their maximum capacity.

Figure 3-1: Theoretical Framework

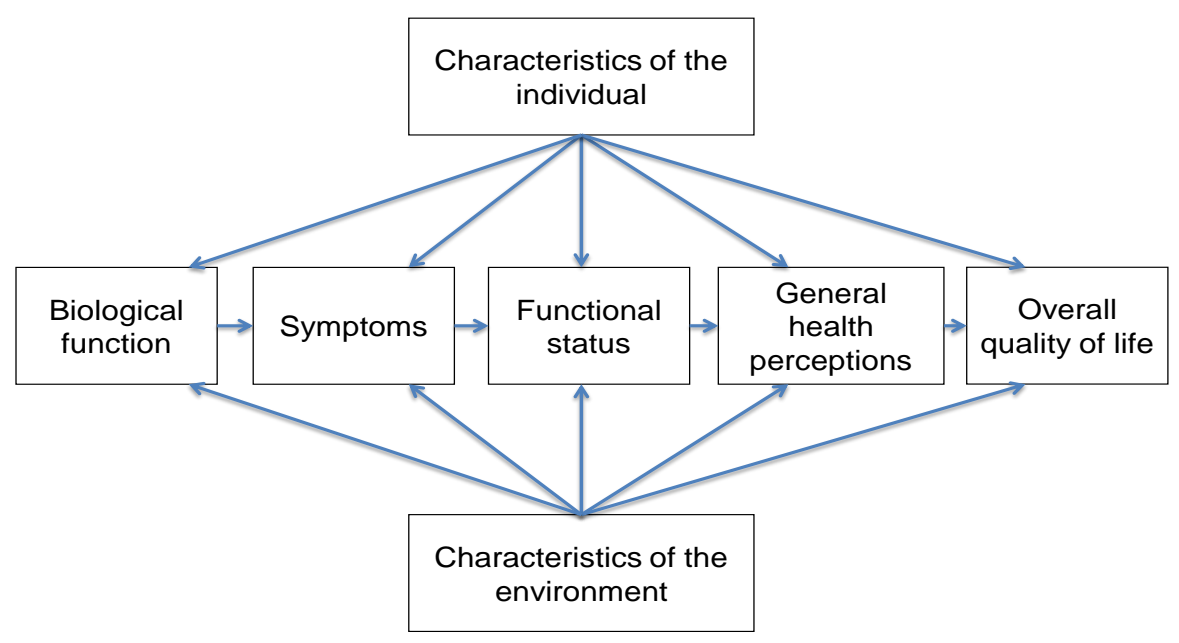

Note: Revised Wilson and Cleary model for health-related quality of life. Adapted from "Linking Clinical Variables with Health-Related Quality of Life: A Conceptual Model of Patient Outcomes," (Wilson \& Cleary, 1995)

In this study, a gait speed assessment was used to measure an individual's physical capacity. Elements of cognitive capacity such as memory and orientation were measured by the MMSE. Functional performance includes activities which are performed daily as a basic necessity of life but are often influenced by personal choice, motivation and self-imposed limits (Ferrans et al., 2005; Leidy, 1994). Instruments such as the Katz Index of Activities of Daily Living (Katz et al., 1963) and Lawton and Brody Scale of Instrumental Activities of Daily Living (Lawton \& Brody, 1969) are commonly used to measure one's ability to independently complete activities of daily living which demonstrates level of functional performance within the functional status realm. Functional reserve is the difference between an individual's capacity and performance. Capacity utilization refers to the ability of an individual to call upon functional potential to perform daily activities (Leidy, 1994). 
By delineating the four dimensions of functional status described by Leidy (1994), the intersecting relationships between biological function, symptoms and characteristics of the environment and individual can be identified. These components combine to generate general health perceptions, which in turn contribute to overall quality of life.

To effectively explore changes in functional status of an elderly individual with severe AS, multiple influencing factors must be taken into account. This conceptual model recognizes that individual and environmental characteristics have a complex and multidirectional relationship between symptoms of severe AS, biological functioning and functional status. Exploring these causal relationships and changes to functional status will lead to a better understanding and measureable overall quality of life.

\subsection{Research Design}

In this study, a prospective, longitudinal cohort design (Polit \& Beck, 2012) was used to explore change in functional status between the time of eligibility assessment and time of TAVI procedure. The purpose of this study was to compare functional status between two time points and explore the relationship between selected covariates and changes in functional status.

\subsubsection{Setting}

The Vancouver THV program performs approximately 16 TAVI procedures every month at two hospitals in the Vancouver area. After having undergone the THV eligibility assessment and decisions regarding plan of care have been determined, patients are notified by the program nurse coordinator whether they are eligible for TAVI and asked if they are willing and agree to be placed on the waitlist. Patients are then contacted when a surgery date becomes available and a Pre-Admission Clinic (PAC) visit is booked. Patients were typically 
booked for their PAC visit at the hospital in which their TAVI was performed and occurred one to seven days prior to procedure.

Data collection for this study took place in the PAC of both hospitals. The assessment of functional status was completed by the researcher at the time of PAC visit (Time-2) and was compared to the global functional assessment completed by the Vancouver THV nurse coordinator at the time of eligibility assessment (Time-1).

\subsubsection{Sampling Plan}

This study used convenience sampling which seeks to include the most convenient or readily available group of potential participants (Polit \& Beck, 2012). The sample population were patients with severe AS who were eligible for TAVI and were ready, willing and able to undergo the procedure. Exclusion criteria were: patients undergoing TAVI in conjunction with another surgical procedure, non-English speaking individuals, participants who scored less than 25/30 on the MMSE at the time of eligibility assessment by the Vancouver THV clinic (Time-1) and participants who scored less than 20/30 on the MMSE during the assessment of functional status by the researcher (Time-2).

Recruitment of participants entailed several steps. When potential study participants were notified of acceptance by the THV clinic and agreed to undergo TAVI, their name was placed on the Vancouver THV database report. With assistance from the THV clinic staff, the researcher mailed a study invitation brochure and consent form to potential participants. The consent form was included in this mailing package to provide the potential participants with adequate time to review the risks and benefits associated with the study. If there was no response from the potential participants within two weeks of receiving the study brochure, 
the researcher conducted a phone call to answer any questions and inquire about potential interest in participation.

In the absence of a pilot study or any prior research examining the relationship between functional status change and severe AS, the effect size was estimated to be large. Using a power analysis table to test for difference of means with a two-tailed test, a sample size of 33 would be required. This calculation was based on an estimated effect size of 0.70 , Power $=.80$ and $\propto=.05$. At completion of this study the total sample size was 32 participants.

\subsubsection{Procedure}

Study participants were assessed at two points in time (Time-1 and Time-2). Time-1 entailed a typical pre-assessment THV clinic visit. The visit involved the standard clinical practice of undergoing a variety of diagnostic tests, surgical consultation and clinical assessment. This assessment was completed by the program nurse coordinator using the Triage Functional Assessment form, which includes components of physical, cognitive and social functioning (Appendix A). The same data were collected by the researcher at Time-2, which took place during the participant's PAC visit between November 2, 2012 and March 27, 2013. Data were recorded on a case report form developed by the researcher and included Time-1 and Time-2 functional assessment outcomes, demographics, social support information, cardiac co-morbidities and symptoms and echocardiograph findings (Appendix B).

\subsubsection{Data Collection}

Data collection was performed primarily by the study researcher. Prior to the beginning of the study, the THV program nurse coordinator provided the researcher with 
education and instructions related to clinic procedures and methods of conducting the functional assessment. The researcher also observed an eligibility assessment completed during a routine TAVI referral. Data collection for three participants at Time-2 was conducted by a research assistant (RA). Education for the RA was provided by the researcher to ensure consistency and reliability of the data collected.

\subsection{Operationalization of Study Constructs: Measurements of Functional Status}

As previously discussed, this study was guided by the notion that measuring functional status leads to the optimization of function and the ability to perform tasks from a multidimensional approach which includes physical, social and cognitive components. To achieve this global assessment, functional status was operationalized with three different instruments. The MMSE was used to address the cognitive functional capacity of participants. To measure functional performance, the 5-Metre Gait Speed test was utilized, and finally, the Canadian Study of Health and Aging Clinical Frailty Scale which combines an individual's ability to perform daily tasks and activity level were also included.

\subsubsection{Mini Mental State Examination}

It was established in previous chapters that cognitive capacity is an important component of functional status. In 1975, Folstein, Folstein \& McHugh, reported the validity and reliability of an exam they called Mini-Mental State Examination which was designed as a clinical tool which could be used to grade levels of cognitive impairment. Relatively simple to use, they described this tool as mini because it included only eleven questions, took five to ten minutes to conduct and encompassed only cognitive aspects of function and excluded questions regarding mood or affect. 
The MMSE (Appendix C) screening tool consists of eleven questions which address orientation to time and place, short-term memory, calculation, immediate recall, language and construct ability. Each question is allotted a certain number of points and are added together to produce an overall score. The maximum score which can be achieved is thirty and for the purpose of analysis, MMSE was used as a continuous variable. Participants were provided with simple, straight-forward instructions which were repeated if necessary at any point during the test and rationale for using this tool was also provided to patients before it was administered. Throughout the test, it was important to maintain a non-stressful environment and good rapport to ensure the participant did not feel a sense of failure if they struggled to provide an answer or were aware of an incorrect response. Assuring the participant that their answers were not used to provide a diagnosis and only to compare to their own score at time of eligibility assessment was also of utmost importance.

In a 2005 study examining the psychometric properties, it was found that the MMSE demonstrated relatively low reliability with multiple cut-off points and lack of standardized scores. The researchers did however, report that total scores could still be clinical helpful if true score confidence intervals were used when interpreting MMSE test scores (Lopez, Charter, Mostafavi, Nibut, \& Smith, 2005). Tombaugh \& McIntrye (1992) reported the reliability and validity of the MMSE to be satisfactory and was successfully able to provide a quantitative assessment of cognitive impairment and captured cognitive changes over time. Reliability was also examined in a study reporting MMSE distribution scores and Crum, Anthony, Bassett \& Folstein (1993) demonstrated a positive association with age and education level. They found the older aged participants and those with less education had lower MMSE scores. This is an important consideration when using this instrument in the 
TAVI population because education levels vary, however, the cohort being studied is within the same reference group of elderly individuals. Results of the study by Crum et al. (1993) demonstrate that comparisons of MMSE scores should be within similar references groups but most importantly, the studies of psychometric properties demonstrate MMSE results should be used to identify cognitive dysfunction and not determine a formal diagnosis.

The MMSE is one of the most widely used screening tests for cognitive function and can provide a useful clinical tool to assess mental status changes (Kappetein et al., 2013; Tombaugh \& McIntyre, 1992). To examine how functional status changes between two time points, the MMSE scores were not evaluated to provide a diagnosis but rather used to demonstrate changes in score over time.

\subsubsection{Frailty}

Frailty was assessed using the Canadian Study on Health and Aging (CSHA) Clinical Frailty Scale (Rockwood, 2005). This frailty scale includes nine scores ranging from one being very fit, to nine representing terminally ill and fully dependent or incapacitated (Appendix D). Each point on the frailty scale includes a visualization and brief description of level of activity related to disease process or symptoms which represents an individual within that frailty category (Dalhousie University Faculty of Medicine, 2012). As a component of the measurement of functional status, the CSHA Clinical Frailty Scale provides valuable information regarding abilities and limitations of daily activities. The descriptions provided within the scale contain examples of high level activities which may be affected by the degree of frailty to facilitate the interpretation of the scores.

The earliest version of the CSHA scale was first developed in 1991 as a seventy item frailty index by Rockwood and the CSHA (Rockwood et al., 2005). This index included 
various clinical deficits, cognitive and physical variables, diseases, disabilities, and activities of daily living. Based upon this frailty index, a team lead by Rockwood further developed a seven-point frailty scale to measure overall clinical frailty incorporating a self-reported measure of disability, measures of cognitive impairment and presence of disease process (Rockwood et al., 2005). In a study comparing the validity of the Clinical Frailty Scale (CFS) with other validated measurements of frailty, Rockwood et al., (2005) reported the scale to be highly correlated $(r=0.80)$ with the previously developed, seventy-point frailty index. In 2008, this CFS was further revised by the CSHA to include two additional points which included; very severely frail and terminally ill. The CSHA CFS is a relatively new instrument, and its psychometric properties are not fully established. There is significant interest among clinicians in the use of the scale because of the simplicity and ease of use and potential impact of interpretation. This nine-point CFS was used to measure frailty in this study.

An assessment of frailty using the CFS is able to provide a measurement of functional performance by reporting day-to-day activities the participant is capable of, as well as the extent of disease burden or symptoms which may cause limitations. A frailty score between one and nine was documented for each study participant and was analyzed as a continuous variable.

\subsubsection{Gait Speed}

Gait speed refers to the time in seconds it takes to walk a pre-determined distance. The use of gait speed to measure an elderly individuals' functioning is emerging as a valid and reliable tool to assess functional status and as a predictor of mortality and morbidity, especially in the elderly population undergoing invasive cardiac procedures and (Afilalo et 
al., 2010; Green., 2012). The 5-Metre Gait Speed test is currently used by the Vancouver THV clinic and was subsequently chosen for this study to assess the functional capacity component of functional status.

In 2010, Afilalo et al. found the 5-Metre Gait Speed test to be a predictor of mortality and morbidity in a cardiac surgery population. In a 2009 literature review, van Kan et al. (2009) demonstrated gait speed to be a predictor of cognitive impairment disability, and mortality. Gait speed as a predictor of survival in older adults was also reported in a large cohort study of geriatric patients (Studenski et al., 2011). Both of these studies demonstrate gait speed to be a valid instrument in predicting outcomes in an elderly population.

In an unobstructed hallway, a five-metre distance was measured and marked. Participants began at the 0-Metre marking and were asked to walk at their usual, comfortable pace past the 5-Metre marking. If participants routinely use a walking aid such as cane or walker, they were permitted to use it during the test. A stopwatch was used to time the distance it took from first step after the 0-Metre marking until first step after the 5-Metre line. If capable, participants were asked to perform the test three times; all sets of times were documented. For statistical analysis, the average of the three times was used as the outcome variable. Gait speed was measured in seconds and analyzed as a continuous variable.

It was noted that variability in gait speed could exist between two time points depending on additional co-existing conditions which may hinder mobility. Other factors such as length of time already spent in PAC, anxiety related to the upcoming procedure or current perception of symptoms may affect reliability. Participants were encouraged to recall their awareness of 'usual pace' when Time-1 assessment occurred in an attempt to ensure similar objectivity with walking pace. 


\subsection{Operationalization of Study Constructs: Predictor Variables}

One of the goals of this study was to explore the influencing factors contributing to changes in functional status while waiting for TAVI. Using the conceptual framework described by Ferrans et al. (2005), multiple factors such as biological function, symptoms, characteristics of the individual and environment can affect functional status. To operationalize these factors, predictor variables were assumed to have causal relationship with changes in functional status. We hypothesized that wait times, age, sex, procedure, living situation, left ventricular ejection fraction and NYHA classification were variables of interest in the study of change in functional status.

\subsubsection{Time Waiting for TAVI}

Time waiting was reported in total days. As discussed earlier, individuals were accepted and placed on the waitlist for TAVI when the decision to proceed with the procedure was recommended by the THV team and the patient accepted to be placed on the wait list. This decision is based upon the eligibility assessment which includes multiple diagnostic tests, appointment with the surgeon, global functional assessment conducted by the THV nurse and a consensus decision by the Heart Team (which includes cardiologists, cardiac surgeons and nurses) about the patient's likelihood to obtain significant benefit from the procedure. For this study, wait-time was defined as the time from eligibility assessment (Time-1) until the time of data collection of functional status assessment conducted by the researcher (Time-2).

\subsubsection{Age}

Age was reported in years. Severe AS typically presents in older adults, therefore, treatment with TAVI is primarily indicated in the elderly population (Holmes et al., 2012). 
The range of the participants' age in this study was between 64 and 93 years, and was therefore useful to explore as a covariate of functional status change. Ferrans et al. (2005) describes age as a characteristic of the individual and although this demographic factor is unchangeable, the information obtained regarding age as a covariate of functional status change may provide clinically relevant interventions targeted to specific age categories. The age of each participant was analyzed as a continuous variable.

\subsubsection{Sex}

Sex was documented as male or female and analyzed as a categorical variable.

\subsubsection{Procedure}

TAVI procedures at the Vancouver THV clinic are currently performed using a transfemoral or transapical approach. For this study, procedure was dichotomized into transfemoral or transapical and analyzed as a categorical variable. As described in Section 1.2, the transfemoral approach uses the femoral artery to access the aortic valve with a catheter based stent valve. When a transapical approach is performed, the transcatheter valve is inserted via a small incision on the left side of the chest. The decision to proceed with transfemoral or transapical approach is made by the multi-disciplinary THV team and is based upon results of the patient's diagnostic films and reports completed during TAVI assessment.

\subsubsection{Living Situation}

The data collection process and baseline characteristics included obtaining specific living situations which included; living alone, living with spouse, in an assisted-living facility or lives with another adult. For the purpose of analysis however, data were dichotomized into 'lives alone' or 'lives with another adult' and analyzed as a categorical variable. The 
conceptual model guiding this study describes characteristics of the environment as having potential influence on functional status and health outcomes. In this aging TAVI population, many individuals are in living situations in which they live alone, live with an adult child or in long-term care facilities. As previously reported, living alone can have adverse health outcomes in this elderly population. Therefore, exploration of living situation as a predictor of change in functional status was considered important to this study.

\subsubsection{Left Ventricular Ejection Fraction}

The left ventricular ejection fraction (LVEF) was collected with baseline data from the patient chart and described in the baseline characteristics as a mean percentage. Biological function can be a critical patient measured outcome which can affect the individual, symptom burden and ultimately functional status. The LVEF is a critical measurement of cardiac functioning and most commonly reported in an echocardiography or cardiac catheterization report. Below normal ejection fraction can be an indication of reduced heart function and is a co-morbid condition associated with worse outcomes of physical and mental health, vitality and quality of life following TAVI (Holmes et al., 2012).

To explore the correlation between LVEF and functional status changes between Time-1 and Time-2, LVEF was dichotomized into two groups; less than $40 \%$ and more than $41 \%$. Those with an LVEF of more than $41 \%$ were considered to have heart function ranging from mildly impaired to normal. An LVEF of less than $40 \%$ may indicate damage due to many attributable causes (American Heart Association, 2013).

\subsubsection{Symptoms}

For this study, the NYHA classification tool was used to measure symptoms as a predictor variable. The symptom burden of an individual with severe AS can also be greatly 
affected by disease process, associated co-morbidities, and characteristics of the individual and environment. As reported previously, the most common symptoms include angina, shortness of breath, fainting and heart failure. The NHYA classification has frequently been used as a validated measurement tool of functional class or category of heart failure. The NHYA has four categories of heart failure ranging from I to IV. Patients are categorized based on the ability to perform physical activity and the extent to which symptoms of heart failure such as shortness of breath, fatigue and angina limit activity level. The NYHA classification is reported by the physician as part of the cardiac assessment. For analysis, NYHA was categorized into two separate groups; people with NYHA class I and II (none to mild symptoms and mild limitations), and people with NYHA class III and IV (symptoms ranging from marked to severe limitations due to symptoms of heart failure) (Bennett et al., 2002).

\subsection{Data Analysis}

Analysis began with screening and cleaning of data to check for errors and perform corrections as needed. After the data were cleaned, descriptive statistics were performed to ensure the variables had not violated any assumptions underlying the statistical analyses which were planned. Paired sample $t$-tests were used to explore changes to the 5-Metre Gait Speed test, CFS and MMSE scores from the time of eligibility assessment to time of procedure. To examine the correlation between the selected categorical covariates with the mean differences in gait speed from Time-1 to Time-2, independent sample $t$-tests were used. A Pearson's $r$ was used to examine the association between age and the mean differences in gait speed. Age was used as a continuous variable. Mann-Whitney $U$ tests were used to examine associations between selected categorical covariates with the mean differences in 
CFS and MMSE scores because data were found to be non-parametric. Spearman's rho was used to analyze the relationship between the mean differences in CFS and MMSE scores and age.

\subsection{Ethical Considerations}

Approval for this study was granted from The University of British Columbia Providence Heath Care Research Ethics Boards (REB) and Vancouver Coastal Health Research Institute. Potential participants received the consent form in the mail in order to provide sufficient time to review before considering whether or not to participate in the study. The consent process occurred in-person between the researcher and participant. The study was described in further detail, and all risk and benefits fully explained. Participants were also informed that participation was voluntary and they could withdraw at any time. Consent was fully informed and ensured the dignity and autonomy of the participants was upheld. In this study, there were no personal risks to the participant but there were also no personal benefit.

To ensure privacy and confidentiality, all personal information was removed after data collection and each participant received a non-identifying code for study analysis. The data were kept in a password protected computer and locked office at the study site. Data will be kept for up to five years and will then be destroyed in a manner consistent with the Research Ethics Board policy.

This chapter has provided a review of the Wilson and Cleary (1995) theoretical framework which was used to guide this study. A thorough description of the study methods, instrumentation used to measure functional status, predictor covariates and ethical considerations were described. 


\section{Chapter 4: Results}

This chapter begins with a description of the study recruitment and enrolment of participants. Characteristics of participants were analyzed including demographic data, reported symptoms and cardiac comorbidities. Study findings including functional status changes from time of eligibility to time of procedure and correlations between selected covariates and mean difference of 5-Metre Gait Speed test, CFS and MMSE are reported.

\subsection{Study Recruitment and Enrolment}

Seventy-six patients were waiting for TAVI between November 5, 2012 and March 27, 2013 (L. Achtem, personal communication, March, 2013) (see Figure 4-1). A total of 22 patients waiting for TAVI were deemed ineligible because they were non-English speaking $(n=3)$, had an MMSE score less than 25 at their time of eligibility assessment $(n=10)$ or did not have Time-1 data $(n=9)$. Although there were 62 eligible patients for this study, a total of 32 were enrolled and completed the study. Six patients were still waiting for TAVI at the time of study completion, six patients refused to participate, two patients died while on the wait list, and eight patients were not contacted. These latter patients were not contacted because three had already attended their PAC visit prior to the researcher being notified, three were in-patients at other facilities and two patients were not enrolled due to the researcher being unaware of their PAC appointment. The final sample size was 32 . 
Figure 4-1: Flow Diagram of Patient Enrolment

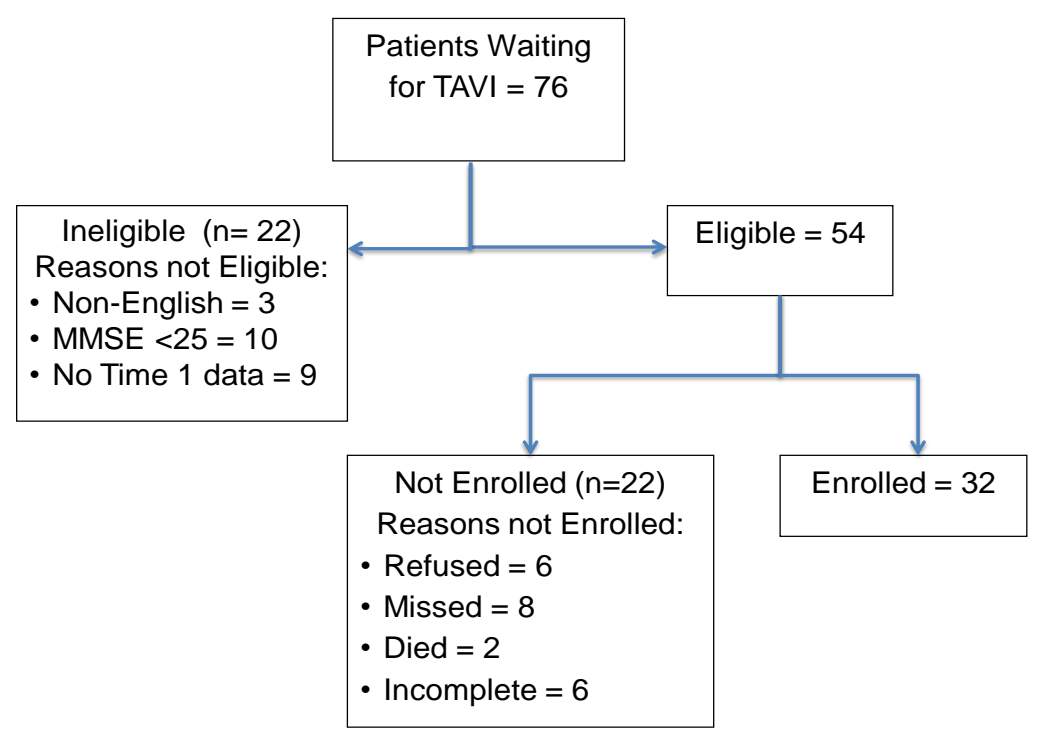

\subsection{Baseline Characteristics}

The average age of the participants was 81 years $(S D=8$, range 64 to 93$)$ with slightly less than half represented by women $(n=14,44 \%)$. Participants waited an average of 69 days ( $S D=62$, range 13 to 307 , median 55) from the time of their eligibility assessment until time of TAVI. More than three quarters of the participants underwent TAVI with the transfemoral approach $(n=25,78 \%)$ with the remaining participants having undergone the transapical approach $(n=7,22 \%)$ (see Table 4-1).

Fifty-six percent of the participants reported being married $(n=18)$ and these participants were all living with their spouses. Sixteen percent of participants were single and $28 \%$ were widowed. Two participants (6\%) were residing in long-term care facilities, $34 \%$ reported they lived alone $(n=11)$, and one participant lived with an adult child and their family. The geographic location of residence was divided equally between participants who lived within Vancouver and those who lived further than $100 \mathrm{~km}$ away. 
Table 4-1: Baseline Characteristics $(N=32)$

\begin{tabular}{|c|c|}
\hline Variable & Total participants \\
\hline Female, $n(\%)$ & $14(44)$ \\
\hline Age (years), mean $\pm S D$ & $81 \pm 8$ \\
\hline Days waiting for TAVI, mean $\pm S D$ & $69 \pm 62$ \\
\hline \multicolumn{2}{|l|}{ Procedure, $n(\%)$} \\
\hline Transapical & $7(22)$ \\
\hline Transfemoral & $25(78)$ \\
\hline \multicolumn{2}{|l|}{ Marital status, $n(\%)$} \\
\hline Married & $18(56)$ \\
\hline Single & $5(16)$ \\
\hline Widowed & $9(28)$ \\
\hline \multicolumn{2}{|l|}{ Living situation, $n(\%)$} \\
\hline Alone & $11(34)$ \\
\hline Facility & $2(6)$ \\
\hline Other adult & $1(3)$ \\
\hline Spouse & $18(56)$ \\
\hline \multicolumn{2}{|l|}{ Residence, $n(\%)$} \\
\hline$>100 \mathrm{~km}$ from Vancouver & $16(50)$ \\
\hline Vancouver & $16(50)$ \\
\hline \multicolumn{2}{|l|}{ New York Heart Association, $n(\%)$} \\
\hline II & $14(44)$ \\
\hline III & $16(50)$ \\
\hline IV & $2(6)$ \\
\hline Syncope, $n(\%)$ & $3(9)$ \\
\hline Angina, n (\%) & $9(28)$ \\
\hline \multicolumn{2}{|l|}{ Mitral regurgitation, $n(\%)$} \\
\hline Mild & $15(47)$ \\
\hline Moderate & $5(16)$ \\
\hline Coronary artery disease, $n(\%)$ & $21(66)$ \\
\hline Previous revascularization, $n(\%)$ & $8(25)$ \\
\hline Atrial fibrillation, $n(\%)$ & $7(22)$ \\
\hline COPD, $n(\%)$ & $10(31)$ \\
\hline LVEF (\%), mean $\pm S D$ & $54 \pm 14$ \\
\hline
\end{tabular}

$\mathrm{NYHA}=$ New York Heart Association; COPD= Chronic obstructive pulmonary disease; Previous revascularization includes Coronary artery bypass graft and/or Percutaneous coronary intervention; LVEF=Left ventricular ejection fraction

Note: \% rounded to the whole number 
Half of the participants $(n=16)$ were reported as having NYHA class II symptoms of heart failure while two (6\%) had class IV symptoms and the remaining had class III symptoms. Only three (9\%) of the participants reported any syncope episodes and nine (28\%) reported symptoms of angina. Additional valve functioning obtained from echocardiogram results showed almost half of the participants ( $n=15,47 \%$ ) had documented mild mitral regurgitation and $16 \%$ had moderate regurgitation $(n=5)$. Two thirds of participants had coronary artery disease $(n=21,66 \%)$ and $25 \%$ had previously undergone revascularization including coronary artery bypass grafting or percutaneous coronary intervention (Coronary bypass surgery: $n=5$; Percutaneous coronary intervention: $n=4$ ). Atrial fibrillation was reported in $22 \%(n=7)$ of the participants and $31 \%(n=10)$ had a documented history of chronic obstructive pulmonary disease. Finally, the average measurement of left ventricular ejection fraction as reported from echocardiogram was $54 \%(S D=14)$.

Overall, this population was elderly, represented nearly equally by men and women, and those living with another adult was nearly twice $(n=21)$ that of participants who lived alone $(n=11)$. Sixteen $(50 \%)$ of the participants were NYHA class III indicating that their symptoms of heart failure markedly limited their daily activities and felt short of breath with minimal exertion. In addition, coronary artery disease was reported in nearly three quarters of the participants $(n=21)$.

\subsection{Primary Research Question}

In individuals with severe AS, does functional status change from the time of eligibility assessment for TAVI until the time of procedure? 
To address this question, results of the 5-Metre Gait Speed test, CFS and MMSE scores of Time- 1 and Time- 2 were compared using a paired $t$-test for statistical analysis and are reported in Table 4-2.

\subsubsection{Functional Status Changes}

The final sample size for the 5-Metre Gait Speed test was 29. Three patients were removed from this analysis for the following reasons: two participants had a difference of more than 4.5 seconds between Time- 1 and Time- 2 which was considered likely erroneous and was attributed to the possibility of inter-rater unreliability or inaccurate documentation, and one participant required a wheelchair for mobility and was unable to complete the gait speed assessment.

Participants had an average walk time at Time- 1 of 7.0 seconds $(S D=2.8)$. At Time-2, the average walk time increased to 7.5 seconds $(S D=2.9)$ with a mean difference of -0.53 . The time it took to walk 5 metres at Time-2 increased demonstrating statistically significant results $(t=-2.75, d f 28, p=0.01,95 \% \mathrm{CI}-0.93$ to -0.14$)$.

Analysis of the CFS was performed yielding an average score at Time-1 of 4.3 (SD= $0.8)$ on a scale of one to nine $(N=32)$. As discussed previously, the CFS ranges from 'one' being a very fit individual, to 'nine' representing an individual who is terminally ill. The average score of the participants at Time-2 was $4.6(S D=0.76)$ with a mean difference of 0.31. The participant's average CFS at Time-2 rose significantly indicating a worsened level of frailty compared to Time-1. The results of this analysis were statistically significant $(t=-$ 2.74, $d f 31, p=0.01,95 \% \mathrm{CI},-0.54$ to -0.08$)$.

Thirty one participants were included in the analysis of comparing MMSE scores at the two time points. Based on a 30 point score, the average MMSE at Time-1 was reported as 
$28.4(S D=1.5)$ and at Time-2 was $28.2(S D=1.5)$. Statistical significance with this analysis was not met $(t=0.86, d f 30, p=0.39,95 \% \mathrm{CI}-0.31$, to 0.76$)$.

Table 4-2: Functional Status Changes from Eligibility Assessment to Time of Procedure

\begin{tabular}{lccccc}
\hline \multicolumn{1}{c}{ Variable } & Time 1 & Time 2 & $\begin{array}{c}\text { Mean } \\
\text { difference }\end{array}$ & 95\% Cl & p Value \\
\hline 5-Metre walk (sec) mean, $S D$ & $7.0(2.8)$ & $7.5(2.9)$ & -.53 & -.93 to -.14 & 0.01 \\
Frailty, mean, $S D$ & $4.3(.8)$ & $4.6(.7)$ & -.31 & -.54 to -.08 & 0.01 \\
MMSE, mean, $S D$ & $28.4(1.5)$ & $28.2(1.5)$ & .23 & -.31 to .76 & 0.39 \\
\hline
\end{tabular}

Note: 5 -Metre Gait Speed $N=29$, Frailty $\mathrm{N}=32$, MMSE $\mathrm{N}=31$

Results of this analysis showed that the time it took for participants to complete the 5Metre Gait Speed test was significantly longer at Time-2 compared to Time-1. Frailty scores were higher at the second time point indicating a worsened or higher level of frailty. The MMSE scores were the only measure of functional status which showed no change between the two time points (see Figure 4-2)

Figure 4-2: Functional Status Changes

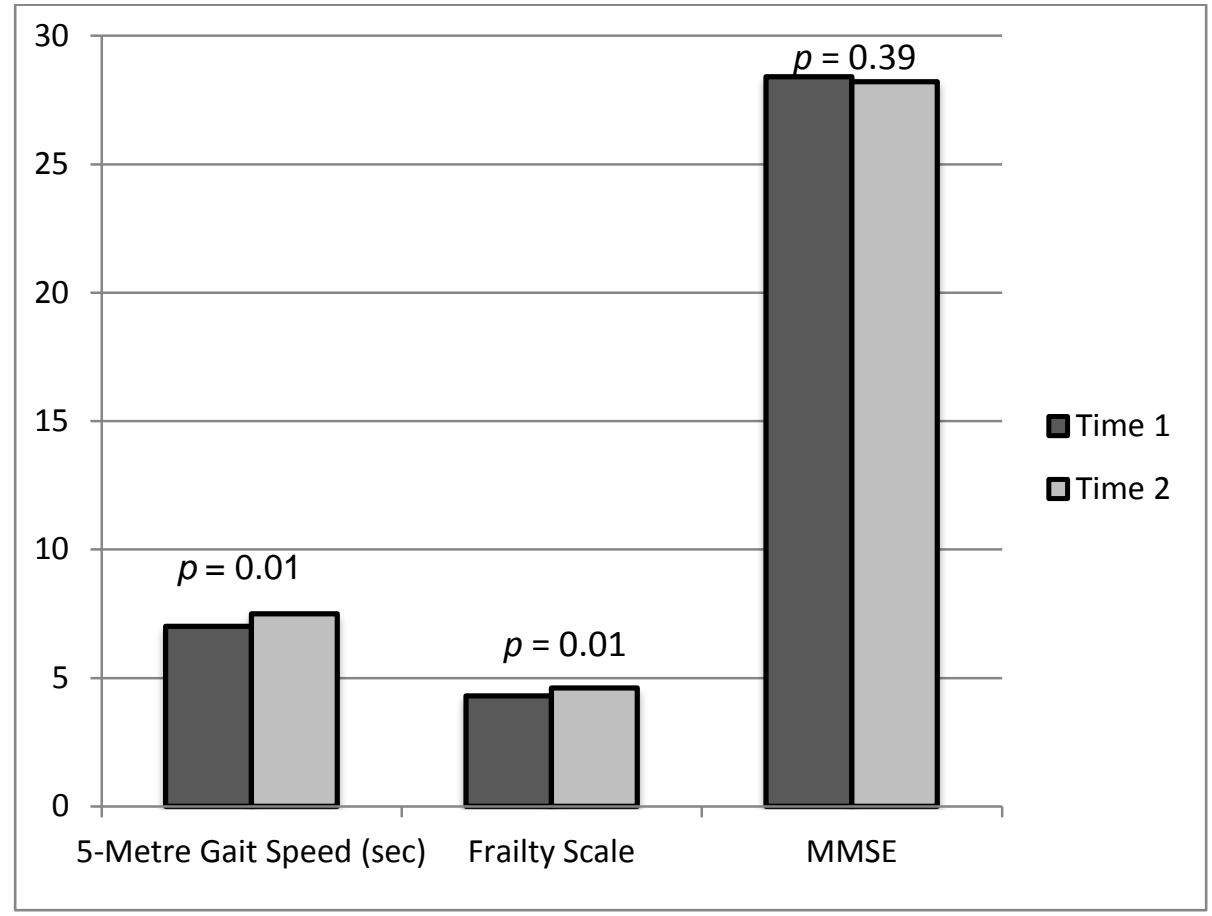

Note: 5 Metre Gait Speed $\mathrm{N}=29$, Frailty Scale $\mathrm{N}=32$, MMSE N=31

Clinical Frailty Scale ranges from very fit (1) to most frail (9), MMSE maximum score 30 points 


\subsection{Secondary Research Question}

What factors are related to changes in functional status in individuals with severe AS who are waiting for TAVI?

To address this question, analyses were conducted to assess the differences between the mean difference of the 5-Metre Gait Speed test, CFS and MMSE by selected predictor variables. The variables explored included: sex, living situation, days spent waiting for TAVI, procedure, left ventricular ejection fraction (LVEF), NYHA classification, and age.

\subsubsection{Mean Differences in 5-Metre Gait Speed Test by Predictor Variables}

As described in Section 3.3.3, the 5-Metre Gait Speed test measured the time in seconds it took for participants to walk a five metre distance. The findings of the mean differences of the 5-Metre Gait Speed by all the predictor variables except age are reported in Table 4-3. Age was analyzed as a continuous variable using a Pearson's $r$ analysis which is reported in Figure 4.2. An alpha level of $<0.05$ was used for all statistical tests. The mean difference in walk time between Time- 1 and Time- 2 showed both male and females to have slower times at the second time point. The mean difference in time for females $(0.8$ seconds, $t=-1.29, d f 27)$, however, was more than twice that of the men $(0.3$ seconds $)$ but the difference between male and females did not show statistical significance $(p=0.21)$.

To explore the correlation between the participants living situation as an independent variable and mean differences in 5-Metre Gait Speed, living situation was dichotomized into two groups: 1) participants who lived with a spouse, another adult or an assisted-care facility, or 2) patients who were living alone. No statistical significance was found with gait speed between Time-1 and Time-2 for those living alone and for participants who lived with another adult, $t=0.17, d f 27, p=0.87$. (see Table 4.3) 
The number of days the participants waited to have their aortic valve implantation was dichotomized into two groups. In the absence of wait-time benchmarks for TAVI, CSBC currently follows the recommended wait-time of six weeks (42 days) utilized for elective patients with severe AS waiting to undergo surgical AVR. For this reason, the first group included participants who had waited between zero and 42 days $(n=10)$, while19 participants waited more than 42 days to have TAVI. Participants who waited 42 days or less had a mean difference of 0 seconds between the two time points and participants who waited more than 42 days showed a mean difference of 0.8 seconds. These absolute values demonstrate that people who waited longer for TAVI had a statistically significant larger change in their gait speed ( $t=-2.13, d f 27, p=0.04,95 \% \mathrm{CI},-1.61$ to -.03 ).

As discussed in previous chapters, participants in this study underwent TAVI using the femoral or transapical approach. Data collection showed TAVI was performed using the femoral approach over three times more often than the transapical approach. The mean difference of change in walk times for participants who underwent TAVI using the transapical approach was over twice as large (1.1 seconds) as those who had the femoral approach (0.4 seconds). Although the difference in mean wait times for these two groups demonstrated a difference of 0.7 seconds, statistical significance was not found $(t=-1.66, d f$ $27, p=0.11,95 \% \mathrm{CI},-1.63$ to 0.17$)$.

Six participants had an LVEF of less than 40\%. These individuals were shown to take longer to walk at Time- 2 than at Time- 1 with a mean difference of 0.9 seconds. Participants with an LVEF of greater than $41 \%(n=23)$ also had slower walk speeds at Time-2 but with a mean difference of only 0.4 seconds. Although statistical significance was not met, these 
results show that participants with poorer cardiac functioning had a bigger change in their walk times $(t=0.99, d f 27, p=0.33,95 \% \mathrm{CI},-0.51$ to 1.46$)$.

The participants' NYHA classification score reporting severity of heart failure symptoms was explored as an independent variable. Thirteen participants were categorized as NYHA I or II and 16 participants were classified with NHYA class III or IV. The mean difference in walk times of participants with NYHA class III or IV was slighter higher (0.6 seconds) than those with class I or II symptoms (0.4 seconds). This shows that participants who were reported by their clinician as having more severe symptoms had a slightly larger change in their gait speed with an absolute mean difference of 0.2 seconds $(t=-0.63, d f 27$, $p=0.54,95 \%$ CI, -1.06 to 0.56 .

Table 4-3: Mean Differences in 5-Metre Gait Speed by Predictor Variable

5-Metre walk test (sec), mean, SD

\begin{tabular}{|c|c|c|c|c|c|c|}
\hline Variable & & Time 1 & Time 2 & $\begin{array}{c}\text { Mean } \\
\text { difference }\end{array}$ & $95 \% \mathrm{Cl}$ & $\begin{array}{c}p \\
\text { Value }\end{array}$ \\
\hline \multirow[t]{3}{*}{ Sex } & Female $(n=12)$ & $8.5(3.8)$ & $9.3(3.6)$ & 0.8 & & \\
\hline & Male $(n=17)$ & $5.9(1.1)$ & $6.2(1.2)$ & 0.3 & & \\
\hline & & & & 0.5 & -1.3 to .30 & 0.21 \\
\hline \multirow{3}{*}{$\begin{array}{l}\text { Living } \\
\text { situation }\end{array}$} & Lives alone $(n=11)$ & $6.3(1.5)$ & $6.9(1.9)$ & 0.6 & & \\
\hline & $\begin{array}{l}\text { Lives with other } \\
\text { adult }(n=18)\end{array}$ & $7.4(3.4)$ & $7.9(3.3)$ & 0.5 & & \\
\hline & & & & 0.1 & -.77 to .90 & 0.87 \\
\hline \multirow{3}{*}{$\begin{array}{l}\text { Days waiting } \\
\text { for TAVI }\end{array}$} & $\leq 42$ days $(n=10)$ & $7.9(2.9)$ & $7.9(3.2)$ & 0 & & \\
\hline & $>42$ days $(n=19)$ & $6.5(2.7)$ & $7.4(2.8)$ & 0.8 & & \\
\hline & & & & 0.8 & -1.61 to -.03 & 0.04 \\
\hline \multirow[t]{3}{*}{ Procedure } & $\mathrm{TA}(n=7)$ & $7.2(1.3)$ & $8.3(1.4)$ & 1.1 & & \\
\hline & $\operatorname{TF}(n=22)$ & $6.9(3.2)$ & $7.3(3.2)$ & 0.4 & & \\
\hline & & & & 0.7 & -1.63 to .17 & 0.11 \\
\hline \multirow[t]{3}{*}{ LVEF } & $<40 \%(n=6)$ & $7.9(4.5)$ & $8.8(4.4)$ & 0.9 & & \\
\hline & $>41 \%(n=23)$ & $6.8(2.3)$ & $7.2(2.4)$ & 0.4 & & \\
\hline & & & & 0.5 & -.51 to 1.46 & 0.33 \\
\hline \multirow[t]{3}{*}{ NYHA } & I-II $(n=13)$ & $5.7(.9)$ & $6.1(1.2)$ & 0.4 & & \\
\hline & III-IV $(n=16)$ & $8.1(3.4)$ & $8.7(3.3)$ & 0.6 & & \\
\hline & & & & 0.2 & -1.10 to .56 & 0.54 \\
\hline
\end{tabular}

Note: $N=29$, LVEF= Left ventricular ejection fraction; NYHA= New York Heart Association 
Figure 4-3: Mean Differences in 5-Metre Gait Speed Test and Age

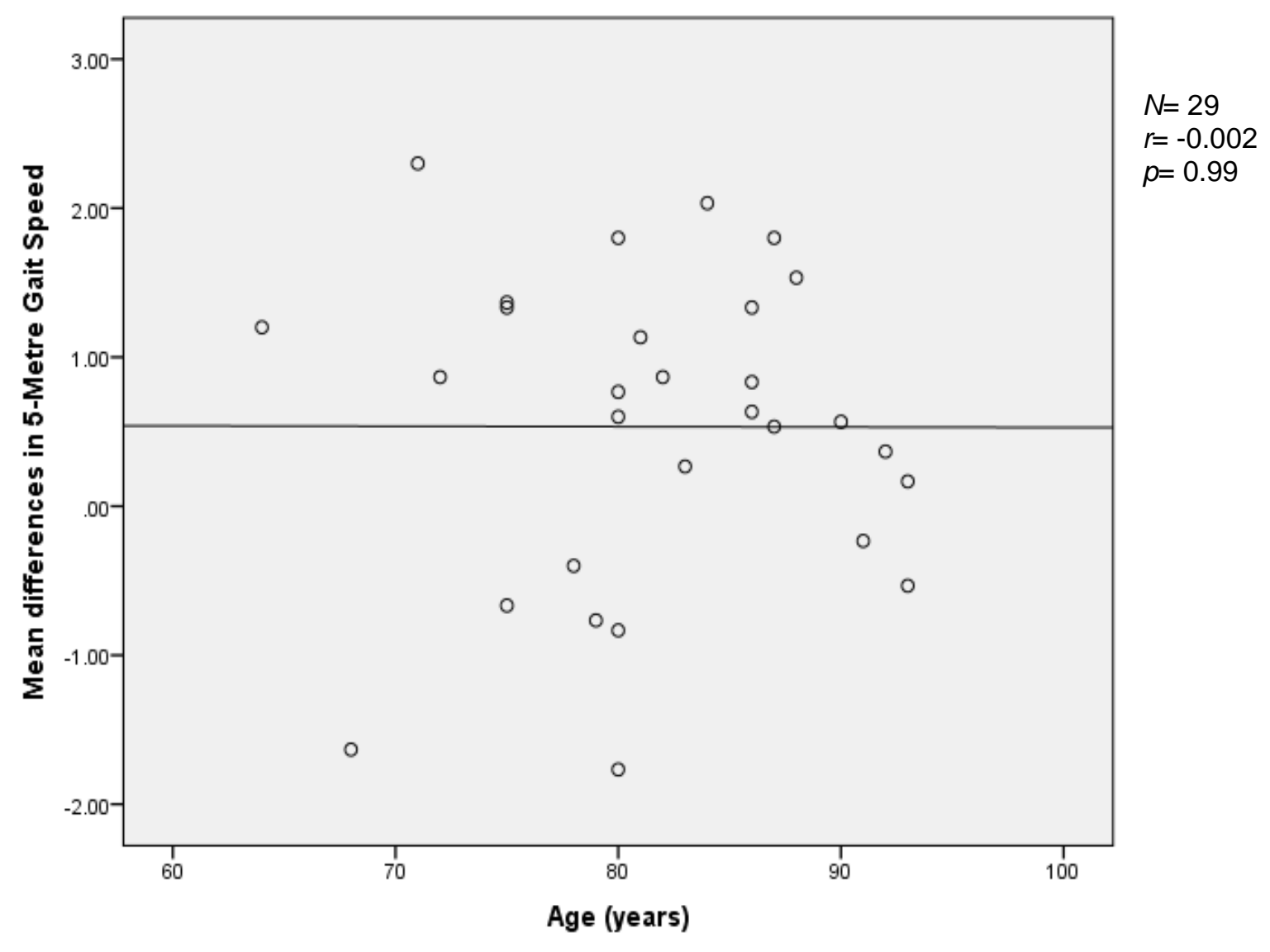

\subsubsection{Mean Differences in Clinical Frailty Scale by Predictor Variables}

Participants' level of frailty was measured using the CFS. This analysis included all 32 participants and was performed using the Mann-Whitney $U$ test because the data did not have a normal distribution. Analysis can be found in Table 4-4. Age was analyzed as a continuous variable using a Spearman rho analysis and is reported in Figure 4.3. An alpha level of $<0.05$ was used for all statistical tests. Both male and female participants had higher frailty scores at Time-2 however; the mean difference between time points for men (0.4) was twice that of women (0.2). The absolute value of the mean difference between men and women was 0.2 with no significant differences noted $(U=105, z=-0.89, p=0.37)$.

There were nearly twice the number of participants who lived with another adult (66\%) compared to those who lived alone (34\%). Both groups had higher frailty scores at 
Time-2 with participants who lived alone having a higher mean difference (0.6) than participants who lived with another adult (0.1). This mean difference was statistically significant, $U=72.5, z=-1.96, p=0.05$ indicating participants who lived alone became more frail and scored higher on the frailty scale while waiting for TAVI.

Participants who waited more than 42 days for TAVI had slightly higher frailty scores at Time-2 with a mean difference of 0.2. Those who waited less than 42 days also had slightly higher frailty scores at Time- 2 with a mean difference of 0.4 . The mean difference between the two groups did not show any significance however, reporting a mean difference of $0.2(U=95.0, z=-1.12, p=0.27)$.

The surgical approach used for TAVI did not show any statistical significance ( $p=$ 0.71 ) between transfemoral or transapical implantation and mean differences in frailty scores. Both groups had slightly higher frailty scores at Time-2 with a mean difference between groups of $0.1, U=80.5, z=-0.37, p=0.71)$.

The majority of participants had a reported LVEF of more than $41 \%(n=25)$ compared to those with an LVEF of less than $40 \%(n=6)$. Both groups were found to have higher frailty scores at Time-2 with those having lower LVEF's having a mean difference of 0.5 and participants with an LVEF above $40 \%$ the mean difference was 0.3 . The mean difference between both groups was not statistically significant $(U=61.5, z=-0.91, p=$ $0.36)$.

The frailty scores at Time-1 were considerably different between participants who were NYHA class I or II (3.7) and those who were NYHA class III or IV (4.7). Participants who were considered to have less limiting symptoms of heart failure with a lower NYHA class were found to have a greater mean difference in their frailty scores between Time- 1 and 
Time-2 (0.6). Participants with NYHA class III or IV had very little change in their frailty scores with a mean difference of 0.1. Although participants with lower NYHA classes had a considerably higher mean difference than those with higher NYHA classification, statistical significance was not reached $(U=82.0, z=-1.92, p=0.06)$.

Table 4-4: Mean Differences in Clinical Frailty Scale by Predictor Variable

\begin{tabular}{|c|c|c|c|c|c|}
\hline \multicolumn{6}{|c|}{$\begin{array}{ll}\text { Frailty } \\
\end{array}$} \\
\hline & Variable & Time 1 & Time 2 & $\begin{array}{c}\text { Mean } \\
\text { difference }\end{array}$ & p Value \\
\hline \multirow[t]{3}{*}{ Sex } & Female $(n=14)$ & $4.6(0.8)$ & $4.9(0.7)$ & 0.2 & \\
\hline & Male $(n=18)$ & $3.9(0.7)$ & $4.3(0.7)$ & 0.4 & \\
\hline & & & & 0.2 & 0.37 \\
\hline \multirow{3}{*}{$\begin{array}{l}\text { Living } \\
\text { situation }\end{array}$} & Lives alone $(n=11)$ & $3.7(0.6)$ & $4.4(0.5)$ & 0.6 & \\
\hline & $\begin{array}{l}\text { Lives with other adult } \\
(n=21)\end{array}$ & $4.5(0.8)$ & $4.7(0.8)$ & 0.1 & \\
\hline & & & & 0.5 & 0.05 \\
\hline \multirow{3}{*}{$\begin{array}{l}\text { Days waiting } \\
\text { for TAVI }\end{array}$} & $\leq 42$ days $(n=12)$ & $4.4(0.9)$ & $4.6(0.8)$ & 0.2 & \\
\hline & $>42$ days $(n=20)$ & $4.2(0.8)$ & $4.6(0.7)$ & 0.4 & \\
\hline & & & & 0.2 & 0.27 \\
\hline \multirow[t]{3}{*}{ Procedure } & Transapical $(n=7)$ & $4.3(0.8)$ & $4.7(0.5)$ & 0.4 & \\
\hline & Transfemoral $(n=25)$ & $4.2(0.9)$ & $4.5(0.8)$ & 0.3 & \\
\hline & & & & 0.1 & 0.71 \\
\hline \multirow[t]{3}{*}{ LVEF } & $<40 \%(n=6)$ & $4.2(1.0)$ & $4.7(1.0)$ & 0.5 & \\
\hline & $>41 \%(n=26)$ & $4.3(0.8)$ & $4.5(0.6)$ & 0.3 & \\
\hline & & & & 0.2 & 0.36 \\
\hline \multirow[t]{3}{*}{ NYHA } & $|-| \mid(n=14)$ & $3.7(0.6)$ & $4.3(0.5)$ & 0.6 & \\
\hline & III-IV $(n=18)$ & $4.7(0.8)$ & $4.8(0.8)$ & 0.1 & \\
\hline & & & & 0.5 & 0.06 \\
\hline
\end{tabular}

Note: $N=32, \mathrm{LVEF}=$ Left ventricular ejection fraction; $\mathrm{NYHA}=$ New York Heart Association

The relationship between age and participants frailty scores was analyzed. Although a small positive correlation was found between the two variables, $r=0.175, p=0.34$ (see Figure 4-4), Table 4-5 reports the change in CFS scores by median age which does not show any relationship. 
Figure 4-4: Mean Differences in Clinical Frailty Scale and Age

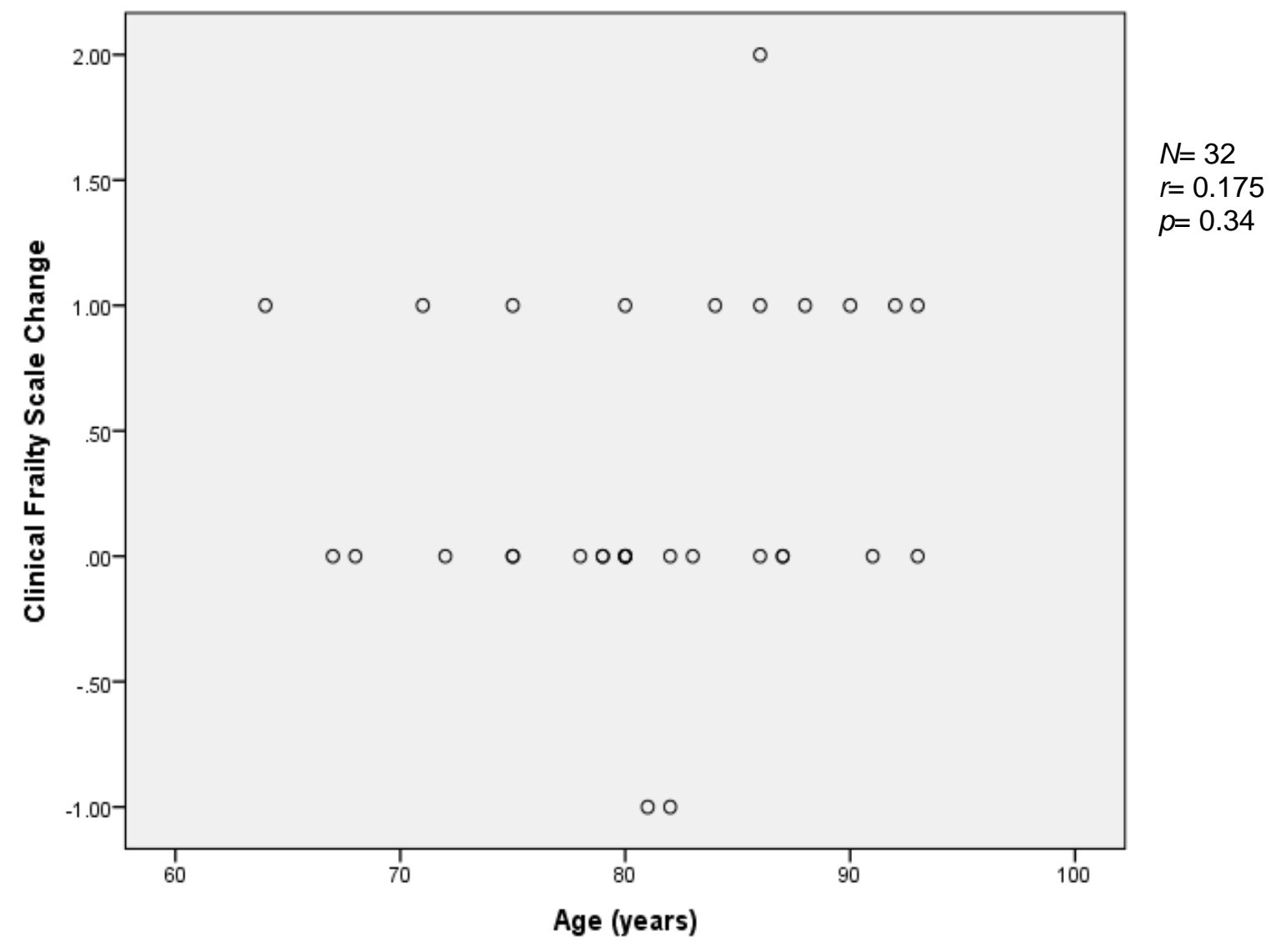

Table 4-5: Changes in Clinical Frailty Scale by Age

\begin{tabular}{lcc}
\hline $\begin{array}{c}\text { Clinical Frailty Scale } \\
\text { (Change in scores) }\end{array}$ & $\begin{array}{c}\text { Age } \\
\text { (median, range) }\end{array}$ & $\begin{array}{c}\text { Total participants } \\
(N)\end{array}$ \\
\hline 1 point lower & $82(81-82)$ & 2 \\
No change in score & $80(67-93)$ & 19 \\
1 point higher & $85(64-93)$ & 10 \\
2 points higher & $86(\mathrm{n} / \mathrm{a})$ & 1
\end{tabular}

Note: Clinical Frailty Scale ranges from very fit (1) to most frail (9), increase in points indicates worsening condition

\subsubsection{Mean Differences in MMSE Scores by Predictor Variables}

Analysis was conducted to explore the mean differences in MMSE scores between groups of patient factors (see Table 4-6). For this analysis, data were available for 31 patients. An alpha level of $<0.05$ was used for all statistical tests. Females had no difference 
in scores between Time- 1 and Time- 2 with a mean difference of zero and males showed a decrease in score with the mean difference being 0.4. The mean difference between males and females was an absolute value of $0.4(U=98.0, z=-0.85, p=0.39)$.

Participants' living situation was not a significant variable with the mean difference between those who lived alone and those who lived with another adult being 0.7, $U=68.5, z$ $=-1.58,(p=0.11)$. Participants who lived with another adult showed no change in their MMSE scores between the two time points.

There was no relationship between days spent waiting for TAVI and the mean difference in MMSE scores. Those who waited less than 42 days showed a slight increase in score with a mean difference of 0.2 and participant's who waited more than 42 days had a slight decrease in their mean difference between Time- 1 and Time-2 of 0.5, $U=86.5, z=-$ $1.14, p=0.25$. 
Table 4-6: Mean Differences in MMSE Scores by Predictor Variable

\begin{tabular}{|c|c|c|c|c|c|}
\hline \multirow{2}{*}{ Variable } & \multicolumn{5}{|c|}{ MMSE } \\
\hline & & Time 1 & Time 2 & $\begin{array}{c}\text { Mean } \\
\text { difference }\end{array}$ & p Value \\
\hline \multirow[t]{3}{*}{ Sex } & Female $(n=14)$ & $27.7(1.5)$ & $27.7(1.7)$ & 0 & \\
\hline & Male $(n=17)$ & $28.7(1.4)$ & $28.5(1.3)$ & 0.4 & \\
\hline & & & & 0.4 & 0.39 \\
\hline \multirow{3}{*}{$\begin{array}{l}\text { Living } \\
\text { situation }\end{array}$} & Lives alone $(n=10)$ & $28.6(1.9)$ & $28.3(1.2)$ & 0.7 & \\
\hline & $\begin{array}{l}\text { Lives with other adult } \\
(\mathrm{n}=21)\end{array}$ & $28.1(1.4)$ & $28.1(1.7)$ & 0 & \\
\hline & & & & 0.7 & 0.11 \\
\hline \multirow{3}{*}{$\begin{array}{l}\text { Days waiting } \\
\text { for TAVI }\end{array}$} & $\leq 42$ days $(n=12)$ & $28.1(1.1)$ & $28.3(1.5)$ & 0.2 & \\
\hline & $>42$ days $(n=19)$ & $28.4(1.8)$ & $28.1(1.5)$ & 0.5 & \\
\hline & & & & 0.3 & 0.25 \\
\hline \multirow[t]{3}{*}{ Procedure } & Transapical $(n=6)$ & $27.9(2.0)$ & $27.5(1.6)$ & 0.8 & \\
\hline & Transfemoral $(n=25)$ & $28.4(1.4)$ & $28.3(1.5)$ & 0.1 & \\
\hline & & & & 0.7 & 0.2 \\
\hline \multirow[t]{3}{*}{ LVEF } & $<40 \%(\mathrm{n}=6)$ & $28.7(1.2)$ & $28.2(1.5)$ & 0.5 & \\
\hline & $>41 \%(25)$ & $28.2(1.6)$ & $28.2(1.5)$ & 0.2 & \\
\hline & & & & 0.3 & 0.46 \\
\hline \multirow[t]{3}{*}{ NYHA } & $|-I|(n=13)$ & $28.8(1.5)$ & $28.7(0.9)$ & 0.4 & \\
\hline & III-IV (n=18) & $27.9(1.5)$ & $27.8(1.8)$ & 0.1 & \\
\hline & & & & 0.3 & 0.84 \\
\hline
\end{tabular}

$\mathrm{p}$ Values obtained with Mann-Whitney U Test

$L V E F=$ Left ventricular ejection fraction; NYHA= New York Heart Association

Participants who underwent the transapical approach to TAVI demonstrated the greatest decrease in MMSE scores at Time-2 with a mean difference of nearly one point (0.8). Those who underwent the femoral approach had almost no change in scores with a mean difference of $0.1(U=50.0, z=-1.28, p=0.2)$.

There was no difference found in MMSE scores between the two time points for those who had a LVEF greater than $41 \%$. Participants with a LVEF of less than $40 \%$ had a small increase in their MMSE score with a mean difference of 0.5. There was no statistical significance found between these two groups $(U=60.5, z=-0.74, p=0.46)$. 
Figure 4-5: Mean Differences in MMSE Score and Age

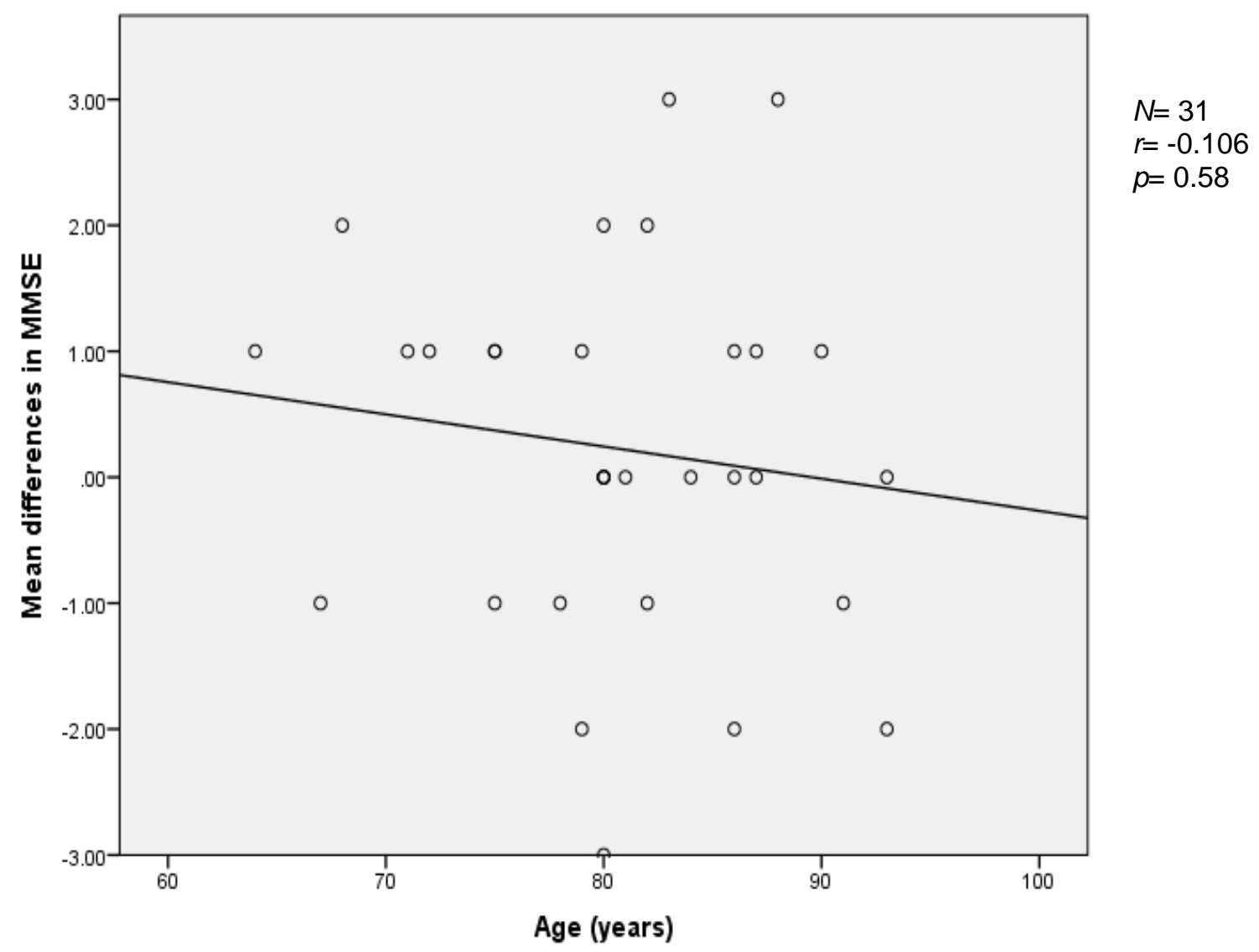

Those who had NYHA class I or II showed a slight decrease in mean MMSE scores with a mean difference of 0.4. No difference was found in those with NYHA class III or IV with a mean difference of $0.1(U=112.0, z=-0.21, p=0.84)$.

To illustrate the relationship between age and the mean difference in MMSE scores, a scatter-plot is shown in Figure 4-5. There was a small, negative relationship found between these two variables with Spearman's $r h o=-0.106, p=0.58$.

\subsection{Summary}

This chapter has presented descriptive statistics for baseline characteristics which included demographic data, symptom burden and cardiac co-morbidities. It also reported results of the analysis used to answer the primary and secondary research questions. 
Results of the changes in CFS and 5-Metre Gait Speed support the primary hypothesis that during the wait time between eligibility assessment and TAVI procedure, individuals with severe AS experience a decline in their functional status.

Results also supported the secondary hypothesis that there is a relationship between select covariates and changes in functional status while waiting for TAVI. Gait speed was slower at Time-2 for all groups of patient factors which were examined. Statistical significance was found with participants who waited for TAVI more than 42 days demonstrated by a slower gait speed at Time-2.. The relationship between the remaining variables and the mean difference in 5-Metre Gait Speed were found to be non-significant however females and participants who underwent femoral TAVI had almost three times larger of a mean difference showing slower walk times at Time-2. In addition, participants who had poorer cardiac functioning as shown by their LVEF and those with NYHA class III or IV also had larger mean differences with slower walk times at Time-2. Participants who lived alone and lived with another adult showed very little difference in their gait speed between Time- 1 and Time- 2 and there was no relationship found between age and mean difference in gait speed.

A significant relationship was found between the mean difference in frailty scores of Time-1 and Time-2 with participants who lived alone. These individuals demonstrated the greatest increase in frailty scores between the two time points compared to participants who lived with another adult. Participants who had lower cardiac functioning with an LVEF of less than $40 \%$ and those who were NYHA classification I or II were also considered to be more frail at Time-2 assessment but results did not show statistical significance. Males had a larger increase in frailty scores than females with the average frailty score rising by 0.4 
points at Time-2. Participants who waited more than 42 days had a slightly higher increase in frailty scores at Time- 2 and very little difference was found between changes in frailty scores between those who had transapical or transfemoral approaches to TAVI.

Analysis of the mean differences of MMSE scores by predictor variables did not demonstrate significant results. The greatest differences between time points with a decrease in MMSE score was found in participants who underwent the transapical approach to TAVI and those who lived alone.

Functional status measured with the 5-Metre Gait Speed test and CFS declined from the time of eligibility assessment until time of procedure, however no significant changes were found using the MMSE. Participants who waited for TAVI more than 42 days were more likely to have the greatest change in gait speed with significantly slower walk times reported at Time-2. Frailty scores were also found to be significantly higher at Time- 2 with those who lived alone indicating a decrease in functional status. 


\section{Chapter 5: Discussion}

The purpose of this study was to describe changes in functional status between the time of eligibility assessment and TAVI procedure date. Using a prospective cohort design, this study provides a description of how functional status changes while waiting for TAVI using the 5-Gait Speed test, CFS and MMSE as measurement tools. In addition, this study examined the correlation between functional status change and specific predictor variables which included; sex, living situation, days waiting for TAVI, procedure, LVEF, and NYHA classification. This chapter provides a summary and discussion of the key findings of this study and an appraisal of study limitations will also be presented. Implications important to nursing practice, leadership, education and recommendations for future research will also be reviewed.

\subsection{Functional Status Changes Waiting for TAVI}

This study demonstrated that elderly patients with severe AS had a decline in their functional status while waiting for TAVI. Functional status measured with the 5-Metre Gait Speed test and CFS showed statistically significant decline from time of eligibility assessment until time of procedure $(p=0.01)$. Cognitive functioning measured by the MMSE did not demonstrate any significant changes. Participants' took longer to walk the five metre distance at Time-2 which indicates a slower gait speed. Frailty scores were higher indicating participants became more frail. The results of two separate measurement tools demonstrating a decline in functional status while waiting for TAVI validate the importance of this study to provide evidence-based best practice. 


\subsubsection{Findings Compared with the Literature}

Scientific evidence has established that severe AS progresses quickly causing symptoms to worsen (Ross \& Braunwald, 1968), however studies examining the effects of the disease trajectory on functional status are limited, and makes this study novel. A study was conducted in 2013 examining the quality of life of patients with severe AS (van Geldorp et al., 2013). Using a patient-reported questionnaire, this study showed patients with severe AS, who had not yet been referred for surgical intervention, had significantly lower physical and social functioning than the general population. The study by van Geldorp et al. (2013) supports the results of this study which showed functional status deteriorates while waiting for TAVI. Providing evidence that gait speed and frailty deteriorate while waiting for TAVI demonstrates the association between the disease progression of AS, worsening symptoms and functional status.

Recognizing a decline in functional status accompanies the downward disease progression of AS, TAVI centres who routinely manage varying wait-times ought to ensure comprehensive functional assessments of their patients to inform thresholds for waiting and pre-procedure interventions. Utilizing the MMSE for cognitive function, 5-Gait Speed to assess exercise capacity and CFS to measure frailty has provided a comprehensive assessment which demonstrated a decline in functional status. Even in the absence of studies specifically examining functional status while waiting for TAVI, this study validates the importance of measuring functional status prior to undergoing TAVI. Afilalo et al. (2010) reported gait speed to be a predictor of mortality and morbidity in cardiac surgery patients and Green et al. (2012) found gait speed and frailty were associated with long-term mortality in an elderly population with severe AS. Although significant results were not found using 
the MMSE, the inclusion of a tool to measure cognitive functioning was in alignment with the study by Schoenenberger et al. (2012) which included the MMSE as a component of their composite frailty index. Contrary to this study which simply compared MMSE scores between two time points, the study by Schoenenberger at al. (2012) dichotomized MMSE into (1) normal cognitive function and (2) cognitive impairment and results showed functional decline after TAVI was associated with pre-operative cognitive impairment. In the near absence of evidence related to functional status changes while waiting for TAVI, this research study provides exploratory and novel results.

\subsection{Correlation between Predictor Variables and Changes in Functional Status}

Predictor variables including; sex, living situation, days waiting for TAVI, procedure, LVEF and NYHA were explored for a relationship with changes in functional status between Time-1 and Time-2. Results of this study have shown individuals who waited longer than 42 days for TAVI had statistically significantly slower gait speed at the time of procedure compared to those who waited less than 42 days. The gait speed for females compared to males and individuals with transapical approach instead of transfemoral TAVI had slower walk times at Time-2 but no statistical significance was found. In addition, we found that individuals who lived alone are likely to become more frail while waiting for TAVI than those who lived with another adult, spouse or in an assisted-living facility. Frailty scores also increased at Time-2 for individuals with NYHA class I and II compared to those with class III and IV but statistical significance was not reached. These results suggest that individuals with poorer symptoms of heart failure actually had a smaller decline in their frailty scores. During the wait time between eligibility assessment and time of procedure, participants who lived alone and waited longer than 42 days experienced the greatest decline in their 
functional status when measured with the 5-Metre Gait Speed and CFS. Comparing the difference between MMSE scores and the selected predictor variables did not yield any statistically significant changes. Individuals who underwent the transapical approach and those who lived alone had slightly lower MMSE scores at Time-2 but were not statistically significant.

\subsubsection{Findings Compared with the Literature}

This study demonstrated functional decline was greater in participants who lived alone compared to those who lived with another adult. Similar results have been shown in the literature such as those described by Mor et al. (1989) who found elderly adults who lived alone were more likely to demonstrate functional decline. Worobey (1990) also reported an increased risk for health problems and institutionalized in older adults who lived alone. Recognizing that older adults living alone are at higher risk to have decline in their functional status is important as our population ages. The 2011 census data provided by Statistics Canada showed that only $21.9 \%$ of people over eighty five years lived with a spouse or common-law partner compared to $70 \%$ of individuals aged 65 to 69 (Government of Canada, 2013). The addition of a chronic disease, such as AS provides increased risks and complications for older adults living alone. In a systematic integrative review, it was reported that older people with chronic illnesses and lived alone were more vulnerable because of limited resources, lack of social support, or challenges faced with making independent decisions regarding management of their disease (Haslbeck, McCorkle, \& Schaeffer, 2012).

There are a number of studies examining mortality and morbidity of patients waiting to undergo aortic valve surgery (Lund et al., 1996; Munt, Humphries, Gao, Moss, \& Thompson, 2006), however exploring functional status while on the wait-list has not been 
found in the literature. In this study, the 5-Metre Gait Speed demonstrated individuals who wait longer than six weeks to undergo TAVI have significantly more decline in their functional status than those who wait less than six weeks. In the absence of specific wait-time benchmarks for TAVI, the CSBC currently uses an Urgency Rating Scale which encompasses all heart surgeries performed in British Columbia. As described in Chapter 1, the Urgency Rating Scale has four categories ranging from (1) Emergency, (2) Priority I, (3) Priority II and (4) Priority III. Individuals with aortic stenosis and the associated symptoms of angina, syncope and heart failure are classified as Priority II with a recommended waittime of six weeks for heart surgery. Priority III includes those deemed to have critical valve disease but stable symptoms and have a recommended wait-time of three months. In 2005, the Canadian Wait Time Alliance also published a report which recommended a six week wait-time for non-urgent patients undergoing valvular heart surgery (Wait-time Alliance for Timely Access to Health Care, 2005). Although the CSBC Urgency Rating Scale and recommendations by the Canadian Wait Time Alliance may be appropriate for other cardiac surgeries including conventional aortic valve replacement, the predictive factors of TAVI such as frailty and gait speed should also be considered when benchmarks are determined. This study has shown elderly individuals undergo functional decline while waiting for TAVI and specific benchmarks for wait-times ought to be developed for this vulnerable population.

\subsection{Findings as they Relate to the Theoretical Framework}

The theoretical framework used to guide this study was the Wilson and Cleary (1995) conceptual model of health-related quality of life modified by Ferrans et al. (2005). This model identifies the complex and multi-directional relationship between five distinct domains and characteristics of the individual and environment. The five domains include; biological 
function, symptoms, functional status, general health perceptions and overall quality of life. Applying the conceptual model to this study, it is apparent the disease pathology of AS, symptom burden and characteristics of the individual and environment have a causal, multidirectional relationship with functional status. This study showed functional status declined while waiting for TAVI which may be attributed to the relationship between disease progression, worsening of symptoms and characteristics of the individual and environment.

The intersecting relationship between functional status and characteristics of the environment were also validated by the results of this study. According to Ferrans et al. (2005), characteristics of the environment includes social components such interpersonal relationships, family and friends. This study found individuals who lived alone had a larger decline in their functional status than those who lived with another adult while waiting for TAVI. As described in Chapter 1, there was a varying wait-time for TAVI at the Vancouver THV clinic and results of this study showed that individuals who waited more than 42 days had significantly slower gait speed with the 5-Metre Gait Speed test than those who waited less than 42 days.

This study suggests functional status can be affected by multiple factors which have been described by the Wilson and Cleary (1995) and Ferrans et al. (2005) conceptual models. Individual's waiting for TAVI have severe AS, additional co-morbidities and significant symptom burden. The addition of multiple individual and environmental considerations create complex, intersecting relationships which ultimately affect functional status as reported in this study. 


\subsection{Limitations}

This study encountered three important limitations which included sample size, reliability and validity of measuring gait speed and exclusion criteria. In Chapter 3, a sample size of 33 was reported as required based on a large effect size. In a study of this size, a small to moderate effect size would have provided stronger correlation among the variables and a larger sample size would help avoid Type II errors. At the onset of the study, it was estimated that approximately 16 TAVI procedures were performed each month by the Vancouver THV program. Unfortunately, data collection extended beyond four months due the number of individuals deemed ineligible. Obtaining a larger sample from all the transcatheter valve clinics across British Columbia would have provided a more representative sample of the population.

Literature has shown gait speed to be a valid measure of functional status and predictor of poor surgical outcomes; however inter-rater reliability and construct validity are possible study limitations. The 5-Metre Gait Speed for two participants reported greater than 4.5 second mean differences between the two time periods with the slower time occurring at the time of eligibility assessment. Based on this finding, these results were considered erroneous and could imply threats to inter-rater reliability or construct validity. Inter-rater reliability refers to the degree in which observations or calculations correlate between two independent observers (Polit, 2010). Even though the researcher was provided thorough instructions from the THV nurse coordinator and observed a routine functional assessment, the procedure for performing the 5-Metre Gait Speed may have been susceptible to inconsistent instructions or calculations. Similarly, construct validity, which Polit (2010) describes as involving "evidence that an instrument is really measuring the underlying 
construct of interest" (pp. 218-219) may also be problematic. The 5-Metre Gait Speed test aims to measure an individual's normal, walking pace to assess functional capacity. However, if an individual misunderstands the instructions, is distracted in any way, or hastily trying to complete the assessment, the measurement of gait speed is not truly measuring ones functional status.

The final limitation of this study was the exclusion criteria determined prior to patient enrollment. As reviewed in Section 3.3.1, the MMSE was used in this study to assess changes in cognitive function between two time points and exclusion criteria included any potential participants' who obtained a score of less than 25 on the MMSE at Time-1.Ten individuals were deemed ineligible to participate because of these criteria. This is considered a limitation because valuable information regarding functional status could have been obtained from these individuals even if the MMSE score truly represented cognitive impairment or was due to their language spoken or their education level. There is varied information in the literature regarding cut-off points for MMSE scores and in some instances, scores are assessed differently depending on age and level of education (Crum et al., 1993; Lopez et al., 2005). Consideration should be given to the notion that individuals are unable to consent to research because of pre-determined MMSE cut-off scores. Taylor, DeMers, Vig \& Borson (2012) reported research conducted by researchers with expertise in gerontology frequently excluded individuals with cognitive impairment even though they are often capable of consenting to research and clinical treatments. In a study exploring functional status changes, a true representation of the population would include those with mild cognitive impairment and an assessment of gait speed and frailty could provide valuable 
information regarding functional capacity and functional performance. The exclusion criteria pre-determined at the onset of this study became its own limitation.

\subsection{Implications}

Exploring functional status of individuals while waiting for TAVI has not previously been studied. The findings of this study have provided important implications to nursing practice, education, policy and recommendations for future research.

\subsubsection{Nursing Practice}

This study demonstrated that functional status declines while waiting for TAVI and a positive relationship exists between functional decline and individuals who live alone and those who wait longer than 42 days. These findings lead to important practice implications for nursing and the multidisciplinary team.

Results of this study have shown that the use of multiple instruments to measure functional status ensures a comprehensive, holistic assessment. Without a standard, operational definition of functional status, health care providers ought to ensure that the nursing care and management of an individual's functioning is multi-dimensional. As described in Section 2.2, the term 'functional status' is often used indiscriminately and in the absence of a standard definition, specific interventions are difficult to achieve. Used independently, the CFS only provides a measure of frailty related to the ability to perform activities of daily living due to symptom burden. The 5-Metre Gait Speed provides an assessment of functional capacity and the MMSE was used to measure cognitive impairment. Separately, these instruments measure distinct components of functional status, but combined are able to provide a comprehensive assessment which can improve patient management and help guide treatment options. Clinicians may be challenged with the decision of choosing 
multiple measures to assess functional status rather than depending on one single measurement tool.

The reliability and validity of instruments used to measure functional status are also important to consider. The MMSE has previously been used to measure cognitive function in TAVI patients (Schoenenberger et al., 2012; Stortecky et al., 2012) however; the small sample size and low sensitivity of the MMSE did not produce statistically or clinically important findings. The purpose of using the MMSE for this study was a pragmatic decision reflecting clinical practice, and aimed to provide a measurement of change over time. The scoring system did not accurately reflect clinically meaningful changes. Orientation to specific locations can be irrelevant to individuals who were assisted to attend their clinic visit by a support person and calculation and language may reflect education level. Another validated tool frequently used to assess cognitive impairment is the Montreal Cognitive Assessment (MoCA). This 30-point measurement tool takes slightly longer to administer than the MMSE because it includes a broader range of cognitive domains which include: attention and concentration, executive functioning, memory, language, visuoconstructional skills, conceptual thinking, calculation and orientation (The Montreal Cognitive Assessment, 2013). Studies have shown the MoCA to have higher sensitivity to mild cognitive impairment, assess higher levels of executive functioning, encompass a larger range of cognitive fields and demonstrate high levels of test-retest reliability (Aggarwal \& Kean, 2010; Cameron et al., 2010). Clinically relevant assessments and research generating information could be obtained with an instrument such as the MoCA used to assess functional capacity. 
Results of this study have shown it is important to provide individuals who live alone with the support and assistance to develop strategies aimed at managing their symptom burden and slowing the trajectory of functional decline. For those who live alone, the desire to remain independent and live at home aligns with health-care policy of providing increased home supports but requires improved self-management skills and social support aimed at meeting specific needs of this patient population (Haslbeck et al., 2012). Individuals who are waiting for TAVI are an elderly, vulnerable population. An improved understanding of this population and the variables that are associated with functional decline help guide specific interventions such as providing social support and self-management skills related to symptom burden and activities of daily living while waiting for TAVI. The risk of functional decline while waiting for TAVI can also be decreased by developing nursing care processes which allow for patients to be re-assessed with a clinic visit or telephone check-in when patient wait-time exceeds 42 days.

\subsubsection{Education}

The need to emphasize multi-dimensional functional assessments and develop strategies to support older adults during times of vulnerability is critical to implement in nursing education. Developing educational initiatives to increase the focus of geriatric care to include functional status is especially important in the presence of severe, AS and comorbidities. Care provided to older adults can be individualized based on specific results obtained with a comprehensive functional assessment.

Education is also required at the multi-disciplinary level to ensure all care-providers of TAVI patients are aware of the potential for functional decline for those who live alone and when wait-times exceed six weeks. Results of this study showing functional decline 
while waiting for TAVI validate the importance of ensuring individual's have a primary cardiologist or general practitioner to provide symptom management and facilitate ways to maintain levels of functional status while waiting for TAVI.

Finally, results of this study will contribute to the education which can be provided to patients and support systems while waiting for TAVI. Education related to the signs and symptoms of worsening AS and decreased functional status could prevent additional deconditioning prior to TAVI which will ultimately improve patient outcomes. Development of patient education material ought to include strategies for self-management of their disease and ways to maintain optimal levels of functional status while waiting for TAVI.

\subsubsection{Policy}

An important implication learned from this study supports the need to establish evidence-based benchmarks regarding urgency ratings and wait-times for TAVI patients, including the time required to complete the multiple diagnostic assessments, and the time waiting for the procedure. Similar benchmarks have been established for conventional heart surgery and percutaneous revascularization (Canadian Cardiovascular Society, 2006). As discussed previously, current BC wait-time benchmarks for elective patients with severe AS waiting for surgical AVR is six weeks. Although no mortality was recorded in the study cohort, evidence demonstrating that functional decline is a predictor of mortality and morbidity may indicate that waiting for TAVI may be unsafe for some patients. .

Policy-makers and clinical leaders ought to develop evidence-based benchmarks specific to individuals waiting for TAVI. Wait-time benchmarks help monitor access and outcomes, support the provision of equitable access to care, improve resource management and inform risk stratification methods. The indications for TAVI in lower risk patients 
continues to be explored in research (Webb, 2012) which further supports the development of evidence-based wait-time benchmarks in a rapidly evolving innovative therapy. Our findings may suggest that the TAVI population requires individualized risk stratification to consider the urgency of the procedure, and the inclusion of a comprehensive functional status assessment to inform individual decision and the development of wait-time benchmarks based on the limited epidemiological data currently available. It may be reasonable to adopt the six week BC elective surgical AVR benchmark as a starting point in the evaluation of wait time and urgency rating.

\subsubsection{Recommendations for Future Research}

It is important for TAVI research to focus on functional status as a component of preprocedure assessment, risk stratification and outcome variable. A review of the literature has shown the inclusion of measures of functional status within TAVI research has increased in recent years (Green et al., 2012; Stortecky et al., 2012) however, there is an absence of a standardized definition of functional status and functional components are often not clearly defined. Future research could inform the appropriate measure of functioning which would facilitate outcome evaluation and provide specific strategies for treatment interventions. Research incorporating changes in functional status could provide evidence based data used to develop TAVI wait-time benchmarks. Duplicating this study in a larger sample could validate the exploratory findings in this study. The addition of a qualitative approach to explore patients' perception of their functional status while waiting for TAVI would provide valuable information and inform future research in this new era of the management of structural heart disease. 


\subsection{Conclusion}

The purpose of this study was to explore changes in functional status between the eligibility assessment until time of TAVI. Guided by the Wilson and Cleary (1995) conceptual model of Health-Related Quality of Life, this study found that functional status decline occurs while waiting for TAVI and suggested that people who live alone and those who waited longer than six weeks experienced significantly worse decline. Although a small sample size was used, these results can generate important changes and nursing implications for those undergoing TAVI. Ensuring that all patients undergo the procedure within six weeks of acceptance for TAVI ought to be adopted as a provincial wait-time benchmark. Nursing care processes and educational initiatives for care-providers, patients and families which are aimed at optimizing functional status while waiting for TAVI is imperative. Providing strategies to prevent functional deconditioning while waiting for TAVI will improve over-all well being and lead to improved patient outcomes. In addition, future research in the field of TAVI ought to ensure a comprehensive evaluation of functional status is included in pre-operative assessments, risk stratification and outcome measures. 


\section{References}

Afilalo, J., Eisenberg, M. J., Morin, J. -., Bergman, H., Monette, J., Noiseux, N., . . Boivin, J. (2010). Gait speed as an incremental predictor of mortality and major morbidity in elderly patients undergoing cardiac surgery. Journal of the American College of Cardiology, 56(20), 1668-1676. doi: 10.1016/j.jacc.2010.06.039

Afilalo, J., Karunananthan, S., Eisenberg, M. J., Alexander, K. P., \& Bergman, H. (2009). Role of frailty in patients with cardiovascular disease. The American Journal of Cardiology, 103(11), 1616-1621. doi: 10.1016/j.amjcard.2009.01.375

Aggarwal, A., \& Kean, K. (2010). Comparison of the folstein mini mental state examination (MMSE) to the montreal cognitive assessment (MoCA) as a cognitive screening tool in an inpatient rehabilitation setting. Neuroscience \& Medicine, 1(2), 39-42. doi: $10.4236 / \mathrm{nm} .2010 .12006$

American Heart Association. (2013). Ejection Fraction Heart Failure Measurement. Retrieved from http://www.heart.org/HEARTORG/Conditions/HeartFailure /SymptomsDiagnosisofHeartFailure/Ejection-Fraction-Heart-FailureMeasurement_UCM_306339_Article.jsp

Apostolakis, E., \& Akinosoglou, K. (2007). Reexamining the new york heart association functional classification of heart failure. American Journal of Cardiology, 100(5), 911912. doi: 10.1016/j.amjcard.2007.05.006 
Bagur, R., Rodes-Cabau, J., Dumont, E., De Larochelliere, R., Doyle, D., Pibarot, P., . . . Bertrand, O. F. (2011). Performance-based functional assessment of patients undergoing transcatheter aortic valve implantation. American Heart Journal, 161(4), 726-734. doi: 10.1016/j.ahj.2010.12.024

Bekeredjian, R., Krumsdorf, U., Chorianopoulos, E., Kallenbach, K., Karck, M., Katus, H. A., \& Rottbauer, W. (2010). Usefulness of percutaneous aortic valve implantation to improve quality of life in patients $>80$ years of age. American Journal of Cardiology, 106(12), 1777-1781. doi:10.1016/j.amjcard.2010.08.017

Bennett, J. A., Riegel, B., Bittner, V., \& Nichols, J. (2002). Validity and reliability of the NYHA classes for measuring research outcomes in patients with cardiac disease. Heart \& Lung: The Journal of Acute and Critical Care, 31(4), 262-270. doi: 10.1067 $/ \mathrm{mhl} .2002 .124554$

Boyd, C. M., Weiss, C. O., Halter, J., Han, K. C., Ershler, W. B., \& Fried, L. P. (2007). Framework for evaluating disease severity measures in older adults with comorbidity. Journals of Gerontology Series A-Biological Sciences \& Medical Sciences, 62(3), 286295. doi:10.1016/j.amjmed.2005.01.062

Brooks, D., Solway, S., \& Gibbons, W. J. (2003). ATS statement on six-minute walk test. American Journal of Respiratory and Critical Care Medicine, 167(9), 1287. doi: 10.1164/ajrccm.167.9.950 
Cameron, J., Worrall-Carter, L., Page, K., Riegel, B., Lo, S. K., \& Stewart, S. (2010). Does cognitive impairment predict poor self-care in patients with heart failure? European Journal of Heart Failure, 12(5), 508-515. doi: 10.1093/eurjhf/hfq042

Canadian Agency for Drugs and Technologies Health. (2013). Transcatheter Aortic Valve Replacement. Retrieved from http://www.cadth.ca/products/environmentalscanning/environmental-scans/environmental-scan-39

Canadian Cardiovascular Society. (2006). CCS commentaries on access to care. Universal access, but when? Treating the right patient at the right time. Wait time benchmarks for cardiovascular services and procedures. Retrieved from www.ccs.ca/download /position_statements/CCS_Atlas_for_CCS.pdf

Canadian Cardiovascular Society Access to Care Working Group. (2005) Wait-time benchmarks for cardiovascular services and procedures. Retrieved from http://www.ccs.ca

Cardiac Services British Columbia. (2012). Waitlist management reference manual. Unpublished data

Cribier, A., Eltchaninoff, H., Bash, A., Borenstein, N., Tron, C., Bauer, F., Derumeaux, G.,... Leon,M. B. (2002). Percutaneous transcatheter implantation of an aortic valve prosthesis for calcific aortic stenosis: First human case description. Circulation, 106(24), 3006-3008. doi: 10.1161/01.CIR.0000047200.36165.B8 
Crum, R. M., Anthony, J. C., Bassett, S. S., \& Folstein, M. F. (1993). Population-based norms for the mini-mental state examination by age and educational level. Journal of the American Medical Association, 269(18), 2386-2391. doi: 10.1001/jama. 1993 .03500180078038

Cucinotta, D. (2007). Prevention of pathological aging by comprehensive clinical, functional and biological assessment. Archives of Gerontology and Geriatrics, 44(SUPPL), 125132. Retrieved from http://www.sciencedirect.com.ezproxy.library.ubc.ca/science /journal/01674943/44/supp/S

Dalhousie University Faculty of Medicine. (2012). Geriatric Medicine Research. Research Projects: Clinical Frailty Scale. Retrieved from http://geriatricresearch.medicine .dal.ca/clinical_frailty_scale.htm

Eyler, A. E., Wilcox, S., Matson-Koffman, D., Evenson, K. R., Sanderson, B., Thompson, J., . . Rohm-Young, D. (2002). Correlates of of physical activity among women from diverse racial/ethnic groups. Journal of Women's Health \& Gender-Based Medicine, 11(3), 239-253. doi: 10.1089/152460902753668448

Ferrans, C. E., Zerwic, J. J., Wilbur, J. E., \& Larson, J. L. (2005). Conceptual model of health-related quality of life. Journal of Nursing Scholarship, 37(4), 336-342. doi: 10.1111/j.1547-5069.2005.00058.x

Folstein, M. F., Folstein, S. E., \& McHugh, P. R. (1975). 'Mini mental state'. A practical method for grading the cognitive state of patients for the clinician. Journal of Psychiatric Research, 12(3), 189-198. doi: 10.1016/0022-3956(75)90026-6 
Folstein, M., Anthony, J. C., Parhad, I., Duffy, B., \& Gruenberg, E. M. (1985). The meaning of cognitive impairment in the elderly. Journal of the American Geriatrics Society, 33(4), 228-235. Retrieved from http://psycnet.apa.org.ezproxy.library.ubc.ca /index.cfm?fa=search.displayRecord\&uid=1986-06438-001

Forman, D. E., Rich, M. W., Alexander, K. P., Zieman, S., Maurer, M. S., Najjar, S. S., . . . Wenger, N. K. (2011). Cardiac care for older adults: Time for a new paradigm. Journal of the American College of Cardiology, 57(18), 1801-1810. doi: 10.1016/j.jacc.2011 .02 .014

Fried, L. P., Tangen, C. M., Walston, J., Newman, A. B., Hirsch, C., Gottdiener, J., . . . McBurnie, M. A. (2001). Frailty in older adults: Evidence for a phenotype. J Gerontol A Biol Sci Med Sci, 56, M146-56. doi: 10.1093/gerona/56.3.M146

Garin, O., Ferrer, M., Pont, A., Rue, M., Kotzeva, A., Wiklund, I., . . Alonso, J. (2009). Disease-specific health-related quality of life questionnaires for heart failure: A systematic review with meta-analyses. Quality of Life Research : An International Journal of Quality of Life Aspects of Treatment, Care and Rehabilitation, 18(1), 71-85. doi: 10.1007/s11136-008-9416-4

Georgiadou, P., Kontodima, P., Sbarouni, E., Karavolias, G. K., Smirli, A., Xanthos, T., .. . Voudris, V. (2011). Long-term quality of life improvement after transcatheter aortic valve implantation. American Heart Journal, 162(2), 232-237. doi: 10.1016/j.ahj .2011 .06 .004 
Goncalves, A., Marcos-Alberca, P., Almeria, C., Feltes, G., Hernandez-Antolin, R. A., Rodriguez, E., . . Zamorano, J. L. (2011). Quality of life improvement at midterm follow-up after transcatheter aortic valve implantation. International Journal of Cardiology,162(2), 117-122. doi: 10.1016/j.ijcard.2011.05.050

Gongidi, V., R., \& Hamaty, J., N. (2011). Aortic stenosis: A focused review on the elderly... first in a continuing series. Clinical Geriatrics, 19(3), 19-22. Retrieved from http://www.clinicalgeriatrics.com/articles/Aortic-Stenosis-Focused-Review-Elderly

Gotzmann, M., Bojara, W., Lindstaedt, M., Ewers, A., Bösche, L., Germing, A., . . Mügge, A. (2011). One-year results of transcatheter aortic valve implantation in severe symptomatic aortic valve stenosis. American Journal of Cardiology, 107(11), 16871692. doi: 10.1016/j.amjcard.2011.01.058

Government of Canada, Canadian Institutes of Health Research. (2008). Health researchinvesting in Canada. Retrieved from http://www.cihr-irsc.gc.ca/e/37663.html

Government of Canada. (2013). Statistics Canada: Living arrangements of seniors. Retrieved from http://www12.statcan.gc.ca/census-recensement/2011/as-sa/98-312x/98-312-x2011003_4-eng.cfm

Green, P., Woglom, A. E., Genereux, P., Daneault, B., Paradis, J. -., Schnell, S., . . Williams, M. (2012). The impact of frailty status on survival after transcatheter aortic valve replacement in older adults with severe aortic stenosis: A single-center experience. JACC: Cardiovascular Interventions, 5(9), 974-981. doi: 10.1016/j. jcin.2012.06.011 
Green, P., Woglom, A. E., Genereux, P., Maurer, M. S., Kirtane, A. J., Hawkey, M., . . . Kodali, S. (2012). Gait speed and dependence in activities of daily living in older adults with severe aortic stenosis. Clinical Cardiology, 35(5), 307-314. doi: 10.1002/clc.21974

Haslbeck, J. W., McCorkle, R., \& Schaeffer, D. (2012). Chronic illness self-management while living alone in later life: A systematic integrative review. Research on Aging, September(34), 507-547. doi: 10.1177/0164027511429808

Health Canada. (2012). Summary Basis of Decision (SBD) for Edwards Sapien Transcatheter Heart Valve. Retrieved from http://www.hc-sc.gc.ca/dhp-mps/prodpharma/sbd-smd/mdim/sbd_smd_2011-sapien_thv_176966-eng.php

Hogan, D. B., MacKnight, C., \& Bergman, H. (2003). Models, definitions, and criteria of frailty. Aging Clinical and Experimental Research, 15(3), 3-29.

Holmes, D. R.,Jr, Mack, M. J., Kaul, S., Agnihotri, A., Alexander, K. P., Bailey, S. R., . . Weitz, H. H. (2012). 2012 ACCF/AATS/SCAI/STS Expert consensus document on transcatheter aortic valve replacement. Catheterization \& Cardiovascular Interventions, 79(7), 1023-1082. doi: 10.1002/ccd.24351

Hunt, S. A., Baker, D. W., Chin, M. H., Cinquegrani, M. P., Feldman, A. M., Francis, G. S., . . Smith Jr, S. C. (2001). ACC/AHA Guidelines for the evaluation and management of chronic heart failure in the adult. Journal of the American College of Cardiology, 38(7), 2101-2113. doi: 10.1016/S0735-1097(01)01683-7 
Kappetein, A. P., Head, S. J., Genereux, P., Piazza, N., Van Mieghem, N. M., Blackstone, E. H., . . Leon, M. B. (2013). Updated standardized endpoint definitions for transcatheter aortic valve implantation: The valve academic research consortium-2 consensus document. Journal of Thoracic and Cardiovascular Surgery, 145(1), 6-23. doi: 10.1016/j.jtcvs.2012.09.002

Katz S., Ford, A. B., Moskowitz, R. W., Jackson, B. A., Jaffe, M. W. (1963). Studies of illness in the aged: The index of ADL: A standardized measure of biological and psychosocial function. The Journal of the American Medical Association, 185(12), 914919. doi: 10.1001/jama.1963.03060120024016.

Knight, M. M. (2000). Cognitive ability and functional status. Journal of Advanced Nursing, 31(6), 1459-1468. doi: http://dx.doi.org/10.1046/j.1365-2648.2000.01446.x

Lally, F., \& Crome, P. (2007). Understanding frailty. Postgraduate Medial Journal, 83, 1620. doi: 10.1136/pgmj.2006.048587

Lawton, M. P., \& Brody, E. M. (1969). Assessment of older people: Self-maintaining and instrumental activities of daily living. Gerontologist, 9(3), 179-186.

Lee, D. H., Buth, K. J., Martin, B., Yip, A. M., \& Hirsch, G. M. (2010). Frail patients are at increased risk for mortality and prolonged institutional care after cardiac surgery. Circulation, 121, 973-978. doi: 10.1161/CIRCULATIONAHA.108.841437

Leidy, N. K. (1994). Functional status and the forward progress of merry-go-rounds: Toward a coherent analytical framework. Nursing Research, 43(4), 196-202. 
Leon, M. B., Piazza, N., Nikolsky, E., Blackstone, E. H., Cutlip, D. E., Kappetein, A. P., . . . Serruys, P. W. (2011). Standardized endpoint definitions for transcatheter aortic valve implantation clinical trials: A consensus report from the valve academic research consortium. European Heart Journal, 32(2), 205-217. doi: 10.1093/eurheartj/ehq406

Leon, M. B., Smith, C. R., Mack, M., Miller, D. C., Moses, J. W., Svensson, L. G., . . PARTNER Trial Investigators. (2010). Transcatheter aortic-valve implantation for aortic stenosis in patients who cannot undergo surgery. The New England Journal of Medicine, 363(17), 1597-1607. doi: 10.1056/NEJMoa1008232

Lopez, M. N., Charter, R. A., Mostafavi, B., Nibut, L. P., \& Smith, W. E. (2005). Psychometric properties of the folstein mini-mental state examination. Assessment, 12(2), 137-144. doi: 10.1177/1073191105275412

Lund, O., Nielsen, T. T., Emmertsen, K., Flø, C., Rasmussen, B., Jensen, F. T., ... Hansen, O. K. (1996). Mortality and worsening of prognostic profile during waiting time for valve replacement in aortic stenosis. Thoracic Cardiovascular Surgery, 44(6), 289-295. doi: $10.1055 / \mathrm{s}-2007-1012039$

Mack, M. J. (2012). Transcatheter aortic valve implantation: Changing patient populations and novel indications. Heart, 98(SUPPL. 4), 73-79.

MacKnight, C., \& Rockwood, K. (2001). Use of the chronic disease score to measure comorbidity in the canadian study of health and aging. International Psychogeriatrics, 13, 137-142. doi: 10.1017/S1041610202008074 
Madden, S., \& Hill, J. (2009). Treatment of older patients with aortic valve stenosis. Nursing Standard, 24(12), 42-48. Retrieved from http://www.nursing-standard.co.uk/

Maddox, G. L. (1987). Aging differently. The Gerontologist. 27(5), 557-564. doi: 10.1093/geront/27.5.557

Maganti, K., Rigolin, V. H., Sarano, M. E., \& Bonow, R. O. (2010). Valvular heart disease: Diagnosis and management. Mayo Clinic Proceedings, 85(5), 483-500. doi: 10.4065 /mcp.2009.0706

Makary, M. A., Segev, D. L., Pronovost, P. J., Syin, D., Bandeen-Roche, K., Patel, P., . . . Fried, L. P. (2010). Frailty as a predictor of surgical outcomes in older patients. Journal of the American College of Surgeons, 210(6), 901-908. doi: 10.1016/j.jamcollsurg .2010 .01 .028

Makkar, R. R., Fontana, G. P., Jilaihawi, H., Kapadia, S., Pichard, A. D., Douglas, P. S., . . Leon, M. B. (2012). Transcatheter aortic-valve replacement for inoperable severe aortic stenosis. New England Journal of Medicine, 366(18), 1696-1704. doi: 10.1056 /NEJMoa1202277

McPherson, B., \& Wister, A. (2008). Aging as a social process: Canadian perspectives (5th ed.). Canada: Oxford University Press.

McRae, M. E., \& Rodger, M. (2012). Transcatheter aortic valve implantation outcomes: Implications for practice. The Journal of Cardiovascular Nursing, 27(3), 270-282. doi: 10.1097/JCN.0b013e318217d288 
Miller-Davis, C., Marden, S., \& Leidy, N. K. (2006). The new york heart association classes and functional status: What are we really measuring?. Heart and Lung: Journal of Acute and Critical Care, 35(4), 217-224. doi: 10.1016/j.hrtlng.2006.01.003

Moinpour, C. M., McCorkle, R., \& Saunders, J. (1988). Measuring functional status. In M. Frank-Stromborg (Ed.), Instruments for nursing clinical research (pp. 23-50) Appleton \& Lange.

Montreal Cognitive Assessment. (2013). Retrieved from http://www.mocatest.org.

Mor, V., Murphy, J., Masterson-Allen, S., Willey, C., Razmpour, A., Elizabeth Jackson, M., . .. Katz, S. (1989). Risk of functional decline among well elders. Journal of Clinical Epidemiology, 42(9), 895-904. doi: 10.1016/0895-4356(89)90103-0

Munt, B. I., Humphries, K. H., Gao, M., Moss, R. R., \& Thompson, C. R. (2006). True versus reported waiting times for valvular aortic stenosis surgery. Canadian Journal of Cardiology, 22(6), 497-502. doi: 10.1016/S0828-282X(06)70267-7

Nkomo, V. T., Gardin, J. M., Skelton, T. N., Gottdiener, J. S., Scott, C. G., \& EnriquezSarano, M. (2006). Burden of valvular heart diseases: A population-based study. The Lancet, 368(9540), 1005-1011. doi: 10.1016/S0140-6736(06)69208-8

Reynolds, M. R., Magnuson, E. A., Wang, K., Thourani, V. H., Williams, M., Zajarias, A., . . . Cohen, D. J. (2012). Health-related quality of life after transcatheter or surgical aortic valve replacement in high-risk patients with severe aortic stenosis: Results from the 
PARTNER (placement of AoRTic TraNscathetER valve) trial (cohort A). Journal of the American College of Cardiology, 60(6), 548-558. doi: 10.1016/j.jacc.2012.03.075

Reynolds, M. R., Magnuson, E. A., Lei, Y., Leon, M. B., Smith, C. R., Svensson, L. G., . . . Cohen, D. J. (2011). Health-related quality of life after transcatheter aortic valve replacement in inoperable patients with severe aortic stenosis. Circulation, 124(18), 1964-1972. doi: 10.1161/CIRCULATIONAHA.111.040022

Reynolds, M. R., Magnuson, E. A., Wang, K., Lei, Y., Vilain, K., Walczak, J., . . Cohen, D. J. (2012). Cost-effectiveness of transcatheter aortic valve replacement compared with standard care among inoperable patients with severe aortic stenosis / clinical perspective. Circulation, 125(9), 1102-1109. doi: 10.1161/CIRCULATIONAHA .111 .054072

Rockwood, K., Stadnyk, K., MacKnight, C., McDowell, I., Hebert, R., \& Hogan, D. B. (1999). A brief clinical instrument to classify frailty in elderly people. The Lancet, 353, 205-206. Retrieved from http://www.thelancet.com/

Rockwood, K. (2005). What would make a definition of frailty successful. Age and Ageing, 34, 432-434. doi:10.1093/ageing/afi146Rockwood, K., Song, X., MacKnight, C., Bergman, H., Hogan, D. B., McDowell, I., \& Mitnitski, A. (2005). A global clinical measure of fitness and frailty in elderly people. Journal of the Canadian Medical Association, 173(5), 489-496. doi:10.1503/cmaj.050051 
Rodes-Cabau, J., \& Mok, M. (2012). Working toward a frailty index in transcatheter aortic valve replacement: A major move away from the "eyeball test". JACC: Cardiovascular Interventions, 5(9), 982-983. doi: 10.1016/j.jcin.2012.07.002

Ross, J. J. M. D., \& Braunwald, E. M. D. (1968). Aortic stenosis. Circulation, 38(1) (SUPPLEMENT V), 61-67. doi: 10.1161/01.CIR.38.1S5.V-61

Rostagno, C., Galanti, G., Comeglio, M., Boddi, V., Olivo, B., Gastone, G., \& Serneri, N. (2000). Comparison of different methods of functional evaluation in patients with chronic heart failure. European Journal of Heart Failure, 2, 273-280. doi: 10.1016 /S1388-9842(00)00091-X

Schoenenberger, A. W., Stortecky, S., Moser, A., Jueni, P., Carrel, T., Huber, C., . . Wenaweser, P. (2012). Predictors of functional decline in elderly patients undergoing transcatheter aortic valve implantation (TAVI). European Heart Journal. 34(9), 684692. doi: 10.1093/eurheartj/ehs304

Severo, M., Gaio, R., Lourenco, P., Alvelos, M., Bettencourt, P., \& Azevedo, A. (2011). Indirect calibration between clinical observers - application to the new york heart association functional classification system. BMC Research Notes, 4(1), 276. doi: $10.1186 / 1756-0500-4-276$

SF-36.org. (2013). A community for measuring health outcomes using SF tools. Retrieved from http://www.sf-36.org/ 
Smith, C. R., Leon, M. B., Mack, M. J., Miller, D. C., Moses, J. W., Svensson, L. G., . . . Pocock, S. J. (2011). Transcatheter versus surgical aortic-valve replacement in high-risk patients. N Engl J Med, 364(23), 2187-2198. doi: 10.1056/NEJMoa1103510

Snyder, C. F., Aaronson, N. K., Choucair, A.,K., Elliott, T. E., Greenhalgh, J., Halyard, M. Y., .. . Santana, M. (2012). Implementing patient-reported outcomes assessment in clinical practice: A review of the options and considerations. Quality of Life Research, 21(8), 1305-1314. doi: 10.1007/s11136-011-0054-х

Stortecky, S., Schoenenberger, A. W., Moser, A., Kalesan, B., Jüni, P., Carrel, T., . . Wenaweser, P. (2012). Evaluation of multidimensional geriatric assessment as a predictor of mortality and cardiovascular events after transcatheter aortic valve implantation. JACC: Cardiovascular Interventions, 5(5), 489-496. doi: 10.1016 /j.jcin.2012.02.012

Studenski, S., Perera, S., Patel, K., Rosano, C., Faulkner, K., Inzitari, M., . . Guralnik, J. (2011). Gait speed and survival in older adults. JAMA - Journal of the American Medical Association, 305(1), 50-58. doi:10.1001/jama.2010.1923

Taylor, J. S., DeMers, S. M., Vig, E. K., \& Borson, S. (2012). The disappearing subject: Exclusion of people with cognitive impairment and dementia from geriatrics research. Journal of the American Geriatrics Society, 60(3), 413-419. doi: 10.1111/j.15325415.2011.03847.x

Thomas, M., Schymik, G., Walther, T., Himbert, D., Lefèvre, T., Treede, H., . . Wendler, O. (2011). One-year outcomes of cohort 1 in the edwards SAPIEN aortic bioprosthesis 
european outcome (SOURCE) registry: The european registry of transcatheter aortic valve implantation using the edwards SAPIEN valve. Circulation, 124(4), 425-433. doi: 10.1161/CIRCULATIONAHA.110.001545

Toggweiler, S., Humphries, K. H., Lee, M., Binder, R. K., Moss, R. R., Freeman, M., .. . Webb, J. G. (2013). 5-year outcome after transcatheter aortic valve implantation. Journal of the American College of Cardiology, 61(4), 413-419. doi: 10.1016/j.jacc .2012 .11 .010

Tombaugh, T. N., \& McIntyre, N. J. (1992). The mini-mental state examination: A comprehensive review. Journal of the American Geriatrics Society, 40(9), 922-935. Retrieved from http://www.cfmal.com/Guardianship\%20related\%20references-1-1110/Tombaugh\%201992\%20The\%20MMSE\%20\%20A\%20comprehensive\%20review.p df

United States Department of Health and Human Services. (2013). Food and Drug Administration, Drug Development Tools Glossary. Retrieved from http://www.fda .gov/Drugs/DevelopmentApprovalProcess/DrugDevelopmentToolsQualificationProgr am/ucm284395.htm

Vahanian, A., Alfieri, O., Al-Attar, N., Antunes, M., Bax, J., Cormier, B., . . Walther, T. (2008). Transcatheter valve implantation for patients with aortic stenosis: A position statement from the european association of cardio-thoracic surgery (EACTS) and the european society of cardiology (ESC), in collaboration with the european association of 
percutaneous cardiovascular interventions. European Heart Journal, 29(11), 1463-1470. doi: 10.1093/eurheartj/ehn183

van Geldorp, M. W. A., Heuvelman, H. J., Kappetein, A. P., Busschbach, J. J. V., Cohen, D. J., Takkenberg, J. J. M., \& Bogers, A. J. J. C. (2013). Quality of life among patients with severe aortic stenosis. Netherlands Heart Journal, 21(1), 21-27. doi: 10.1007 /s12471-012-0364-9

Van Kan, G. A., Rolland, Y., Bergman, H., Morley, J. E., Kritchevsky, S. B., \& Vellas, B. (2009). The I.A.N.A. task force on frailty assessment of older people in clinical practice.12(1), 29-37. doi: 10.1007/BF02982161

Wang, T. (2004). Concept analysis of functional status. International Journal of Nursing Studies, 41(4), 457-462. doi: 10.1016/j.jnurstu.2003.09.004

Webb, J. G. (2012). Mid-term follow-up after transcatheter aortic valve implantation. European Heart Journal, 33(8), 947-948. doi:10.1093/eurheartj/ehs018

Wilson, I. B., \& Cleary, P. D. (1995). Linking clinical variables with health-related quality of life. A conceptual model of patient outcomes. JAMA, 273(1), 59-65. doi: 10.1001/jama. 1995.03520250075037

Worobey, J. L. (1990). Functional capacity and living arrangements of unmarried elderly persons. Journal of Gerontology, 45(3), S95-S101. doi: 10.1093/geronj/45.3.S95 


\section{Appendix A}

\section{HEART CENTRE}

ST. PAUL'S HOSPITAL -

PROVIDENCE HEALTH CARE

Transcatheter Heart Valve Program

TRANSCATHETER HEART VALVE REFERRAL TRIAGE FUNCTIONAL ASSESSMENT

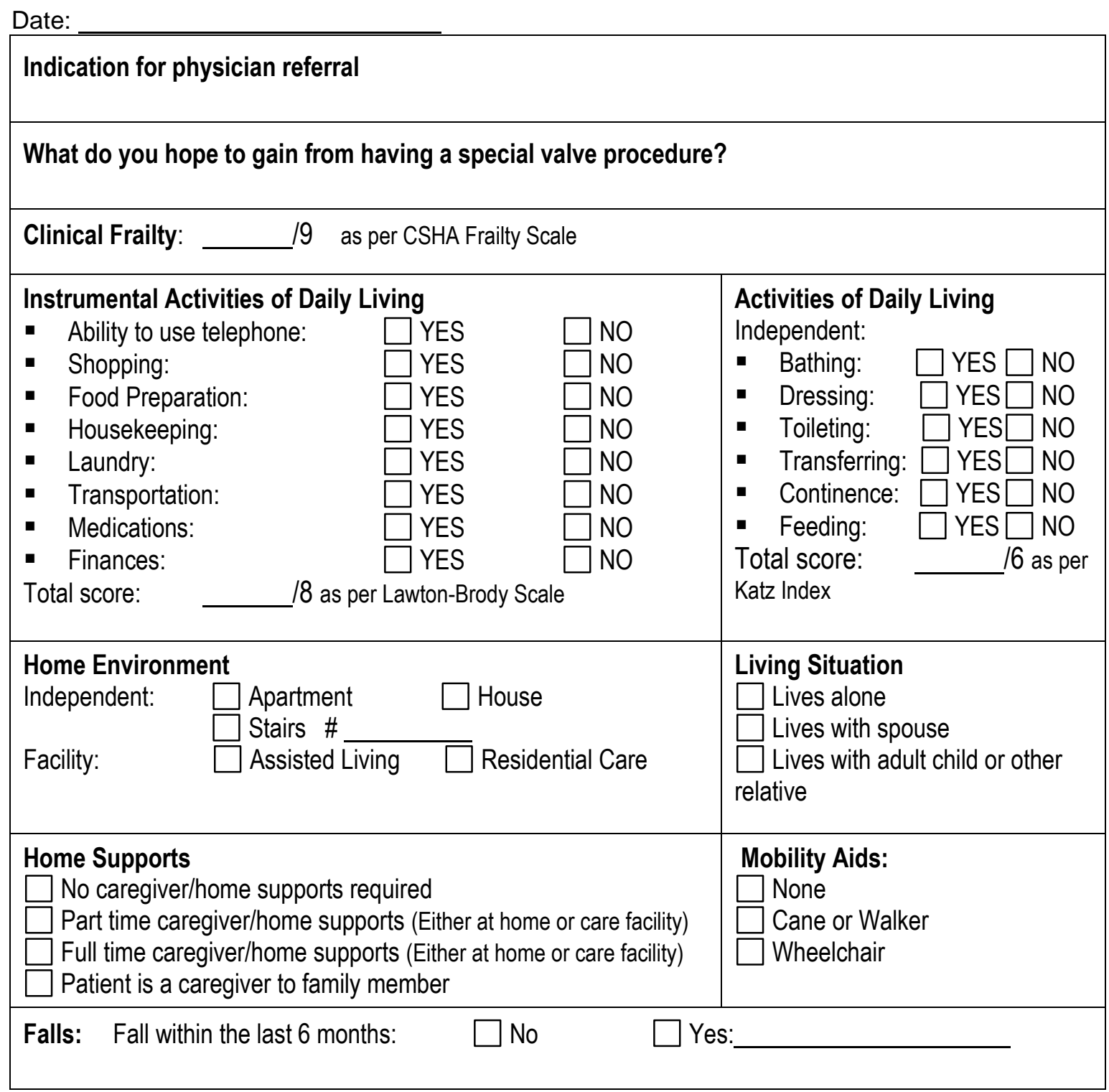




\begin{tabular}{|c|c|}
\hline $\begin{array}{l}\text { 5-Metre Gait Speed Test } \\
\text { > Position the patient with his/her feet behind and just touching the 0-meter start line } \\
>\text { Instruct to "Walk at your comfortable pace" until a few steps past the 5-meter mark } \\
\text { (should not start to slow down before) } \\
>\text { Begin each trial on the word "Go" } \\
>\text { Start the timer with the first footfall after the 0-metre line } \\
>\text { Stop the timer with the first footfall after the 5-metre line }\end{array}$ & 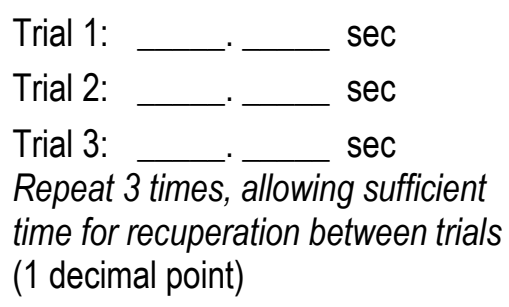 \\
\hline $\begin{array}{l}\text { Cognition - MMSE } \quad \text { /30 } \\
\text { assessment diagram below }\end{array}$ & $\begin{array}{l}\text { Cognition - Clock test } \\
\square \text { Complete: Shape, numbers, } \\
\text { hands, time } \\
\square \text { Unable to complete: Any of the } \\
\text { above missing }\end{array}$ \\
\hline
\end{tabular}

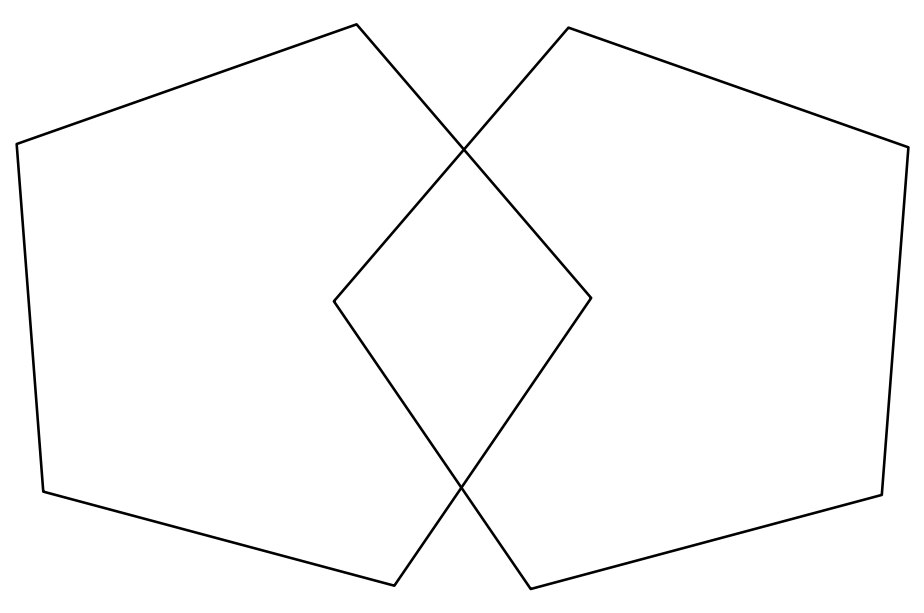

\section{Clock drawing:}

Assessment conducted by: Printed name:

RN Signature: 


\section{Appendix B}

TAVI-FXN Case Report Form

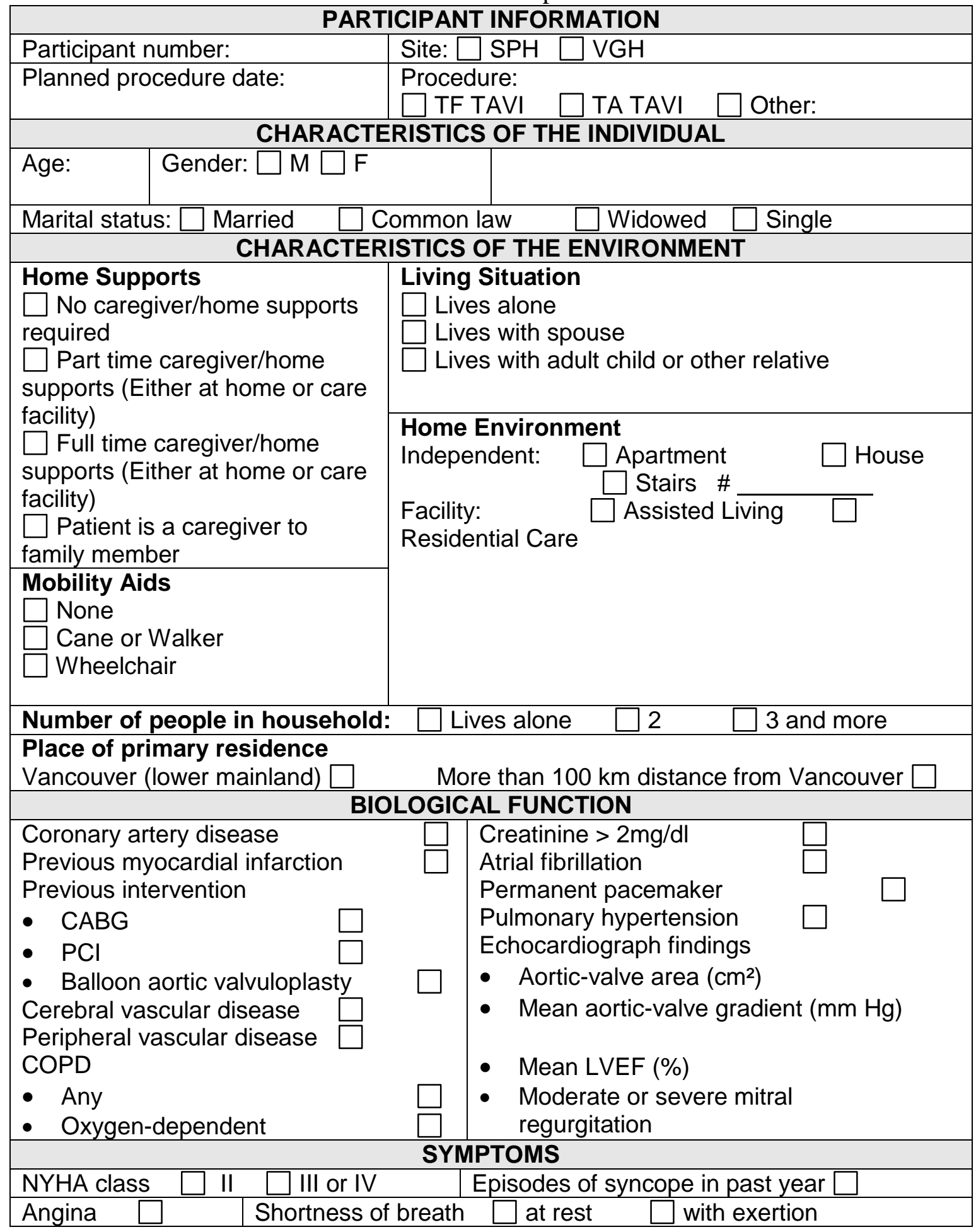




\begin{tabular}{|c|c|}
\hline \multicolumn{2}{|c|}{ FUNCTIONAL STATUS } \\
\hline TIME 1 & TIME 2 \\
\hline Date & Date \\
\hline \multicolumn{2}{|c|}{ FRAILTY } \\
\hline$\square 3 \square 4 \square 5 \square 6$ & $\square 4 \square 5$ \\
\hline $\begin{array}{l}\square 7 \text { Other: } \\
\text { Frailty Scale) }\end{array}$ & $\begin{array}{l}\square 7 \square \text { Other: } \\
\text { Frailty Scale) }\end{array}$ \\
\hline \multicolumn{2}{|c|}{$\begin{array}{l}\text { INSTRUMENTAL ACTIVITIES OF DAILY LIVING } \\
\end{array}$} \\
\hline \multirow{12}{*}{\multicolumn{2}{|c|}{ 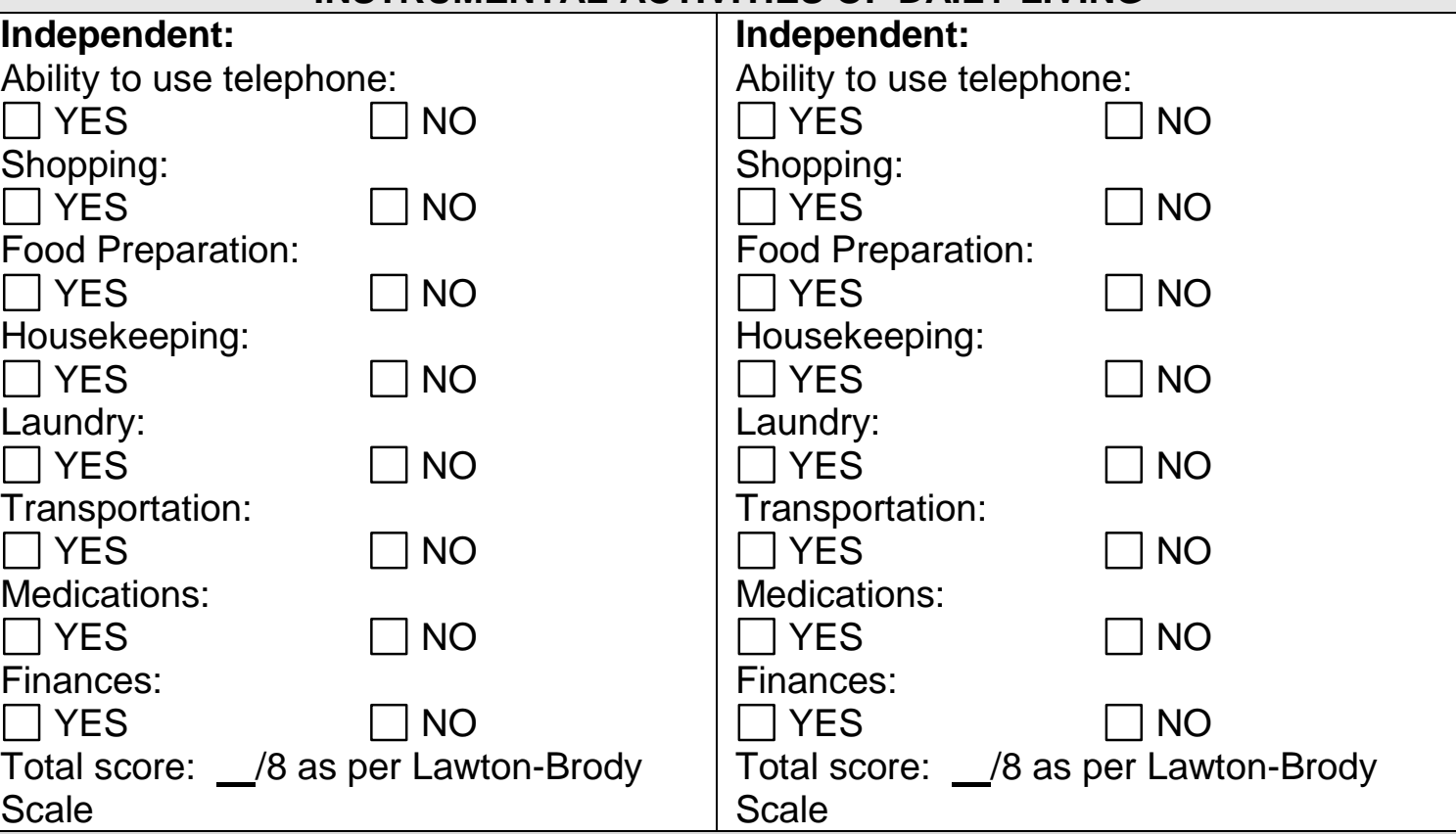 }} \\
\hline & \\
\hline & \\
\hline & \\
\hline & \\
\hline & \\
\hline & \\
\hline & \\
\hline & \\
\hline & \\
\hline & \\
\hline & \\
\hline \multicolumn{2}{|c|}{$\begin{array}{l}\text { ACTIVITIES OF DAILY LIVING } \\
\end{array}$} \\
\hline 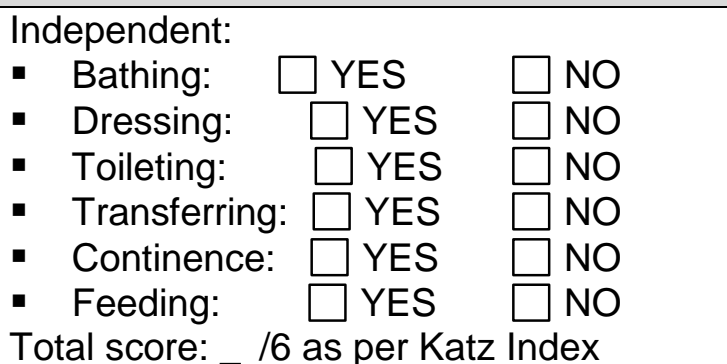 & $\begin{array}{lll}\text { Independent: } & \\
\text { - Bathing: } & \square \text { YES } & \square \text { NO } \\
\text { - Dressing: } & \square \text { YES } & \square \text { NO } \\
\text { - Toileting: } & \square \text { YES } & \square \text { NO } \\
\text { - Transferring: } & \square \text { YES } & \square \text { NO } \\
\text { - Continence: } & \square \text { YES } & \square \text { NO } \\
\text { - Feeding: } & \square \text { YES } & \square \text { NO } \\
\text { Total score: } & 16 \text { as per Katz Index } & \end{array}$ \\
\hline \multicolumn{2}{|c|}{ 5-METRE GAIT SPEED } \\
\hline Trial 1:___ $\_\mathrm{sec}$ & \multirow{3}{*}{ 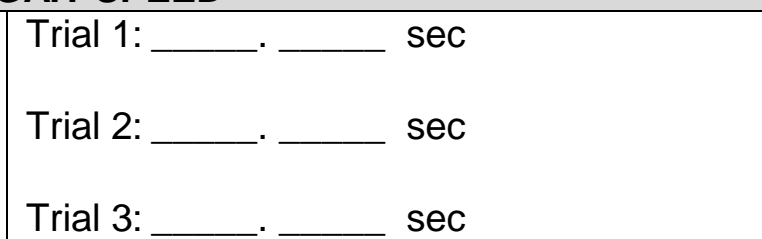 } \\
\hline Trial 2: ___ & \\
\hline rial 3: & \\
\hline \multicolumn{2}{|c|}{ COGNITION } \\
\hline $\begin{array}{l}\text { MMSE } / 30 \text { assessment diagrams below } \\
\text { Clock test } \\
\square \text { Complete: Shape, numbers, hands, } \\
\text { time } \\
\square \text { Unable to complete: Any of the above } \\
\text { missing }\end{array}$ & $\begin{array}{l}\text { MMSE } 130 \text { assessment diagrams below } \\
\text { Clock test } \\
\square \text { Complete: Shape, numbers, hands, time } \\
\square \text { Unable to complete: Any of the above } \\
\text { missing }\end{array}$ \\
\hline
\end{tabular}


Question 10 from MMSE - Write a sentence:

Question 11 from MMSE - copy the design shown
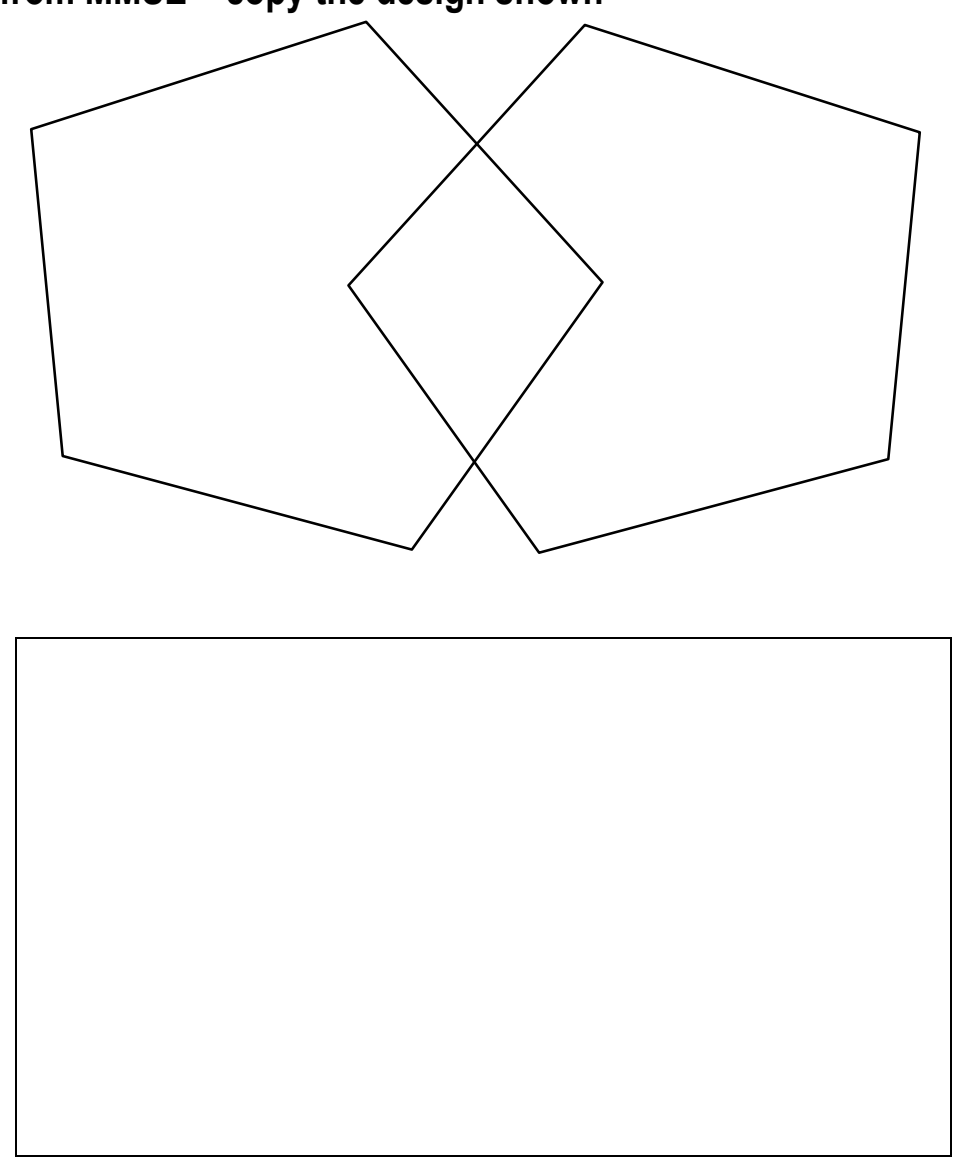

Clock Test:

"Draw a clock put all the numbers in and set the time to 10 past 11 "

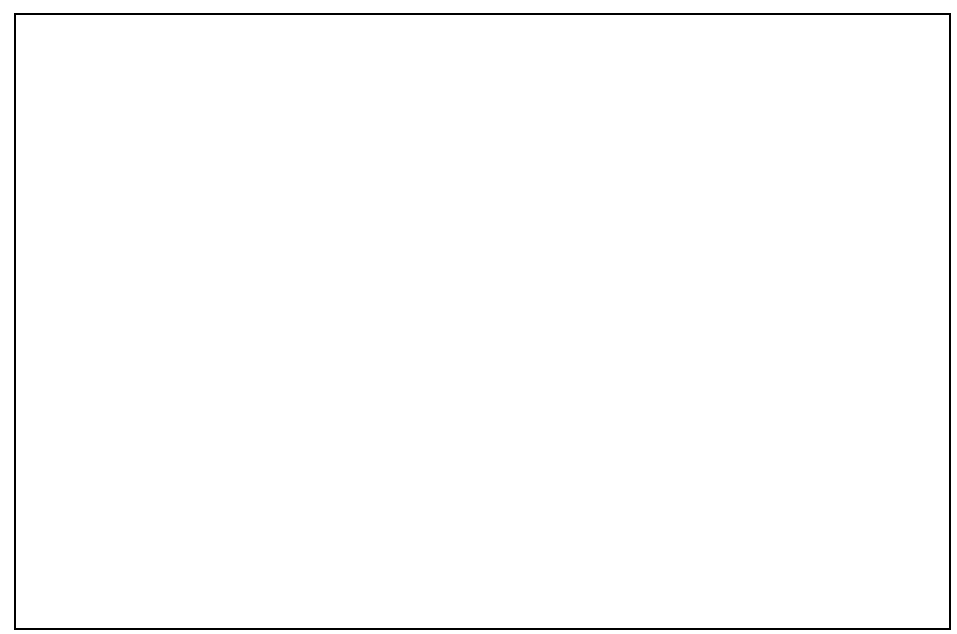




\section{Appendix C}

\section{The Mini-Mental State Exam}

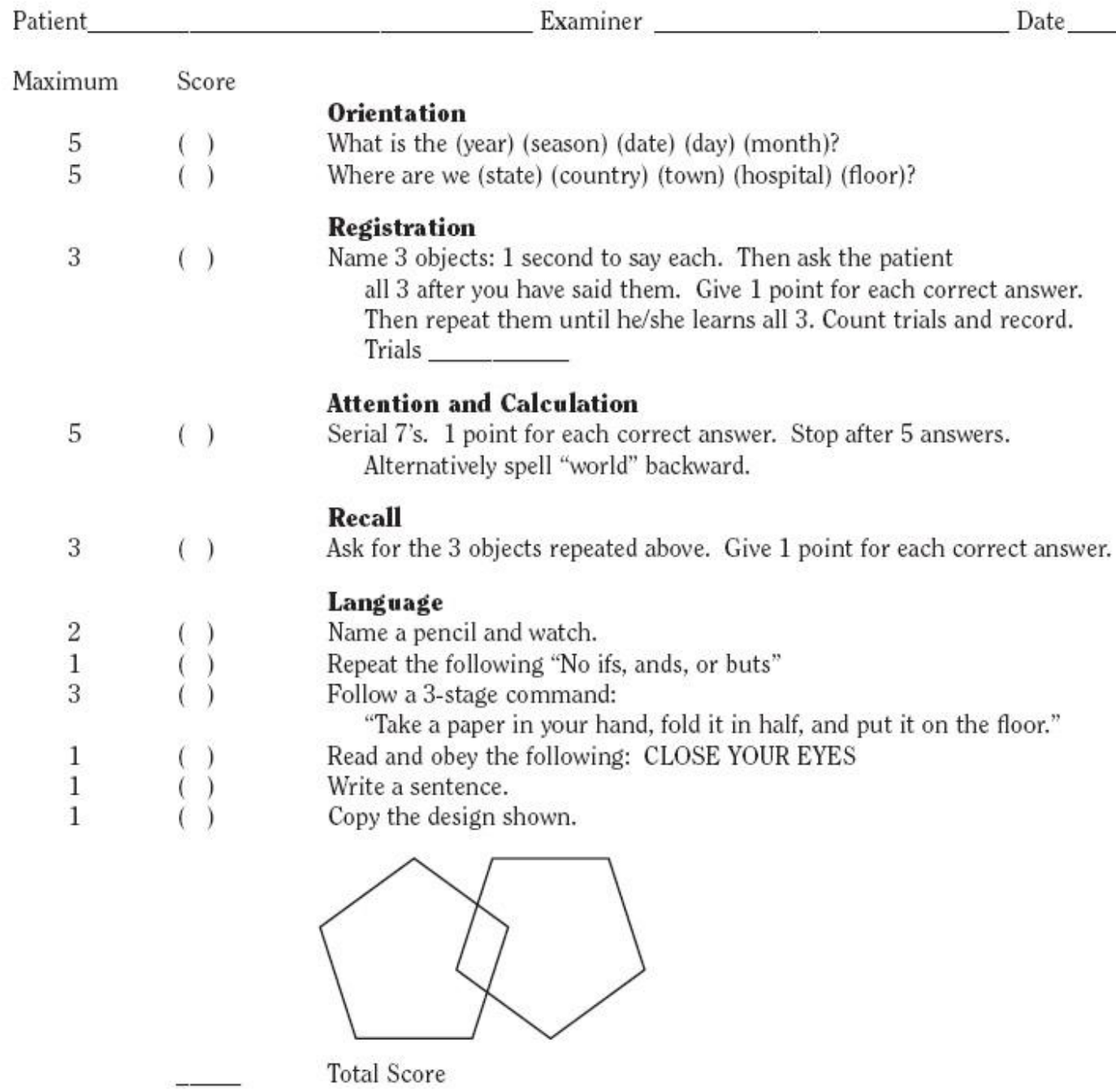




\section{Appendix D}

\section{Clinical Frailty Scale*}

1

Very Fit - People who are robust, active, energetic and motivated. These people commonly exercise regularly. They are among the fittest for their age.

i

2 Well-People who have no active disease symptoms but are less fit than category 1. Often, they exercise or are very active occasionally, e.g. seasonally.

3 Managing Well - People whose medical problems are well controlled, but are not regularly active beyond routine walking.

4 Vulnerable - While not dependent on others for

1 daily help, often symptoms limit activities. A commor complaint is being "slowed up", and/or being tired during the day.

i 5 Mildly Frail - These people often have more evident slowing, and need help in high order IADLs (finances, transportation, heavy housework, medications). Typically, mild frailty progressively impairs shopping and walking outside alone, meal preparation and housework.

6 Moderately Frail - People need help with all outside activities and with keeping house. Inside, they often have problems with stairs and need help with bathing and might need minimal assistance (cuing, standby) with dressing.

\section{S 7 Severely Frail - Completely dependent for personal care, from whatever cause (physical or cognitive). Even so, they seem stable and not at high risk of dying (within $\sim 6$ months).}

8 Very Severely Frail - Completely dependent, approaching the end of life. Typically, they could

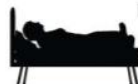

not recover even from a minor illness.

\section{s}

9. Terminally III - Approaching the end of life. This category applies to people with a life expectancy $<6$ months, who are not otherwise evidently frail.

Scoring frailty in people with dementia

The degree of frailty corresponds to the degree of dementia. Common symptoms in mild dementia include forgetting the details of a recent event, though still remembering the event itself, repeating the same question/story and social withdrawal.

In moderate dementia, recent memory is very impaired, even though they seemingly can remember their past life events well. They can do personal care with prompting.

In severe dementia, they cannot do personal care without help.

* 1. Canadian Study on Health \& Aging, Revised 2008. 2. K. Rockwood et al. A global clinical measure of fitness and frailty in elderly people. CMAJ 2005:173:489-495.

() 2007-2009. Version 1.2.All rights reserved. Geniatric Medicine Research, Dalhousie University, Halifax, Canada Permission granted to copy for research and educational purposes onik 